Japan. J. Math.

Vol. 6, No. 2, 1980

\title{
The Cauchy problem for operators with constant coefficient hyperbolic principal part and propagation of singularities
}

\author{
By Seiichiro Wakabayashi
}

(Received July 16, 1979)

\section{Introduction}

Chapter I. Operators with constant coefficients

$\S 1.1$. The Cauchy problem

$\S 1.2$. Hyperbolic polynomials

$\S 1.3$. Generalized wave front sets

$\S 1.4$. Fundamental solutions and singularities of solutions

$\S 1.5$. Some remarks

Chapter II. Operators with variable lower-order coefficients

$\S 2.1$. Construction of solutions

$\S 2.2$. Necessary conditions for well-posedness

$\S 2.3$. Necessary and sufficient condition for $\mathscr{E}$-well posedness

$\S 2.4$. Wave front sets of solutions

Appendix

$\S$ A.1. Procedure to determine the class of hyperbolicity in the case $n \leqq 3$

$\S$ A.2. Examples

\section{Introduction}

Lax [16] and Mizohata [17] proved that for the non-characteristic Cauchy problem to be $\mathscr{E}$-well posed it is necessary that the characteristic roots are real. Moreover Ivrii [11] proved that for the non-characteristic Cauchy problem for operators with analytic coefficients to be well-posed in Gevrey classes it is necessary that the characteristic roots are real. However, in general, the Cauchy problem for operators with hyperbolic principal part is not $\mathscr{E}$-well posed. On the other hand Ivrii [10] proved that the Cauchy problem for such operators is well-posed in some Gevrey classes. So one must consider the Cauchy problem in Gevrey classes (or in the space of ultradistributions) if one imposes no conditions on the lower order terms. In this paper 
we shall study the Cauchy problem for operators with constant coefficient hyperbolic principal part in Gevrey classes (or in the space of ultradistributions).

Let $P(x, D)$ be a partial differential operator of order $m$ in $n$ independent variables, where $x=\left(x_{1}, \cdots, x_{n}\right)$ and $D=i^{-1}\left(\partial / \partial x_{1}, \cdots, \partial / \partial x_{n}\right)$. We assume that

$$
\begin{aligned}
& P(x, D)=P_{m}(D)+Q(x, D), \\
& Q(x, D)=\sum_{j=0}^{m-1} Q_{j}(x, D), \quad Q_{j}(x, D)=\sum_{|\alpha|=j} a_{\alpha}(x) D^{\alpha},
\end{aligned}
$$

where $\alpha$ is a multi-index $\left(\alpha_{1}, \cdots, \alpha_{n}\right)$. Let $K$ be a regular compact set in $\boldsymbol{R}^{n}$ and let $1<\kappa<\infty$ and $h>0$. We denote by $\mathscr{E}^{\{k\}, h}(K)$ the space of all $f \in C^{\infty}(K)$ which satisfies

$$
\left|D^{\alpha} f(x)\right| \leqq C h^{|\alpha|}(|\alpha| !)^{\kappa}, \quad x \in K, \quad|\alpha|=0,1,2, \cdots,
$$

for some constant $C \geqq 0$ and by $\mathscr{D}_{K}^{\{x\}, h}$ the space of all $f \in \mathscr{E}^{\{x\}, h}(K)$ with support in $K$. Then $\mathscr{E}^{\mathscr{R}\{\}\}, h}(K)$ and $\mathscr{D}_{K}^{\{\alpha\}, h}$ are Banach spaces under the norm defined by

$$
\|f\|_{\delta\left\{[\}, h_{(K)}\right.}=\sup _{x \in K, \alpha}\left|D^{\alpha} f(x)\right| /\left(h^{|\alpha|}(|\alpha| !)^{x}\right) .
$$

Let $\Omega$ be an open set in $\boldsymbol{R}^{n}$. As locally convex spaces we define the following:

$$
\begin{aligned}
& \mathscr{E}^{(x)}(\Omega)=\lim _{K \subset \Omega} \mathscr{E}^{(x)}(K), \quad \mathscr{E}^{(x)}(K)=\lim _{h \rightarrow 0} \mathscr{E}^{\{x\}, h}(K), \\
& \mathscr{E}^{\{x\}}(\Omega)=\lim _{K \subset \Omega} \mathscr{E}^{\{x\}}(K), \quad \mathscr{E}^{\{[\}}(K)=\lim _{h \rightarrow \infty} \mathscr{E}^{\{[\}, h}(K), \\
& \mathscr{D}^{(\kappa)}(\Omega)=\underset{K \subset \Omega}{\lim _{K}} \mathscr{D}_{K}^{(\kappa)}, \quad \quad \mathscr{D}_{K}^{(\kappa)}=\lim _{h \rightarrow 0} \mathscr{D}^{\{x\}, h},
\end{aligned}
$$

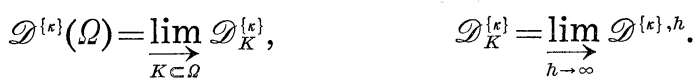

We denote by $\mathscr{D}^{(k)^{\prime}}(\Omega)$ and $\mathscr{D}^{\{\kappa\}^{\prime}}(\Omega)$ the strong dual spaces of $\mathscr{D}^{(x)}(\Omega)$ and $\mathscr{D}^{\{x\}}(\Omega)$, respectively (see [13]). We also write $\mathscr{E}^{(k)}, \cdots$, instead of $\mathscr{E}^{(x)}\left(\boldsymbol{R}^{n}\right), \cdots$, and put

$$
\begin{aligned}
& \mathscr{E}^{(\infty)}(\Omega)=\mathscr{E}^{\mathscr{\infty}\}}(\Omega)=\mathscr{E}(\Omega)\left(=C^{\infty}(\Omega)\right), \\
& \mathscr{D}^{(\infty)^{\prime}}(\Omega)=\mathscr{D}^{\{\infty)^{\prime}}(\Omega)=\mathscr{D}^{\prime}(\Omega),
\end{aligned}
$$

although

$$
\mathscr{E}(\Omega) \neq \bigcup_{x>1} \mathscr{E}^{\mathscr{E}(k)}(\Omega)=\bigcup_{x>1} \mathscr{E}^{[x\}}(\Omega)
$$

Moreover, hereafter, we denote by $*_{\kappa}$ either $(\kappa)$ or $\{\kappa\}$, for example, $\mathscr{E}^{\mathscr{*} *}=\mathscr{E}^{(\kappa)}$ or $\mathscr{E}^{\{k\}}$.

Let us consider the Cauchy problem for $P(x, D)$ 


$$
\left\{\begin{array}{l}
P(x, D) u(x)=f(x), \\
\operatorname{supp} u(x) \subset\left\{x \in \boldsymbol{R}^{n} ; x \cdot \vartheta \geqq t\right\},
\end{array}\right.
$$

where supp $f \subset\left\{x \in \boldsymbol{R}^{n} ; x \cdot \vartheta \geqq t\right\}, t \in \boldsymbol{R}$ and $\vartheta=(1,0, \cdots, 0) \in \boldsymbol{R}^{n}$. If $P(x, D) \equiv$ $P(D)$ is an operator with constant coefficients, then a necessary and sufficient

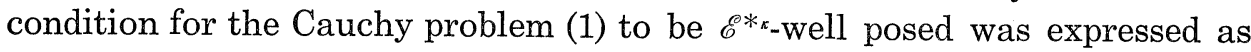
conditions on the zeros of $P_{m}(\xi)$ and $P(\xi)$ (by Gårding [4], [5] for $\kappa=\infty$ and Larsson [15] for $1<\kappa<\infty)$. Moreover Atiyah, Bott and Gårding [1] investigated the singular supports (wave front sets) and the lacunas of fundamental solutions for such operators hyperbolic in the sense of Gårding. On the other hand, for operators with variable lower-order coefficients Dunn [3] proved that the Cauchy problem (1) is $\mathscr{E}$-well posed if

(2) $P_{m}(\xi)$ is a hyperbolic polynomial with respect to $\vartheta$ and $Q(x, \xi) \prec P_{m}(\xi)$ for every fixed $x$ in $\boldsymbol{R}^{n}$

(see, also, [20]), where $q(\xi) \prec p(\xi)$ means that there is a positive number $C$ such that

$$
\tilde{q}(\xi) \equiv\left(\sum_{\alpha}\left|q^{(\alpha)}(\xi)\right|^{2}\right)^{1 / 2} \leqq C \tilde{p}(\xi) \quad \text { for every } \xi \text { in } \boldsymbol{R}^{n} .
$$

Here we have used the notation $q^{(\alpha)}(\xi)=\left(\partial^{\alpha} / \partial \xi^{\alpha}\right) q(\xi)$. From Svensson [19] it follows that the condition (2) is equivalent to the condition

$(2)^{\prime} \quad P(x, \xi)$ is a hyperbolic polynomial with respect to $\vartheta$ in the sense of Gårding for each fixed $x$ in $\boldsymbol{R}^{n}$.

Under the condition (2) the wave front sets of the solutions of the Cauchy problem (1) were studied in [20]. So, in this paper, we shall consider the following; (i) necessity of the condition (2) for the Cauchy problem (1) to be $\mathscr{E}$ well posed, (ii) extension of Svensson's results in $\mathscr{E}^{*}$, (iii) well-posedness of the Cauchy problem (1) in $\mathscr{E}^{* *_{\kappa}}$, and (iv) singularities of the solutions of the Cauchy problem (1) in $\mathscr{E} *$.

In general, the Cauchy problem (1) has no solution in $\mathscr{E}$ (or in $\mathscr{D}^{\prime}$ ) even if $P_{m}(\xi)$ is hyperbolic with respect to $\vartheta$ and $f \in \mathscr{E}$ (or $\left.\mathscr{D}^{\prime}\right)$. However, the solutions of (1) microlocally belong to $\mathscr{E}$ (or $\mathscr{D}^{\prime}$ ) at almost every point in $T^{*}\left(R^{n}\right) \backslash 0$. Let us consider the equation

$$
\left\{\begin{array}{l}
\left(D_{1}^{2}+D_{2}\right) u(x)=\delta(x), \\
\operatorname{supp} u(x) \subset\left\{x \in \boldsymbol{R}^{2} ; x_{1} \geqq 0\right\} .
\end{array}\right.
$$

Then we have

$$
u(x)=-\sum_{j=0}^{\infty}(-i)^{j} x_{1+}^{2 j+1} /(2 j+1) ! \otimes \delta^{(j)}\left(x_{2}\right) \in \mathscr{D}^{(2)^{\prime}},
$$


where $x_{1+}=x_{1}$ if $x_{1} \geqq 0,=0$ if $x_{1}<0$. It can be proved that $u(x)=0$ if $x_{2} \neq 0$ and that $u(x)$ microlocally belongs to $\mathscr{D}^{\prime}$ at $(x, \xi)$ in $T^{*}\left(R^{2}\right) \backslash 0$ if $(x, \xi) \neq\left(\left(x_{1}, 0\right),(0, \lambda)\right)$ for all $\lambda>0$ and $x \neq 0$. We shall show that this result can be extended to the general case.

The remainder of this paper is organized as follows. In chapter I we shall consider operators with constant coefficients, using the same arguments as in [1]. Larsson's result will be stated in $\S 1.1$. The extension of Svensson's result will be given in $\S 1.2$. In $\S 1.3$ generalized wave front sets will be defined, which microlocally characterize singularities with respect to $\mathscr{E} *_{\star}$ and $\mathscr{D}^{* x^{\prime}}$. The outer estimates of the generalized wave front sets of the solutions of (1) will be given in $\S 1.4$. We shall give some remarks on the inner estimates of the generalized wave front sets of the fundamental solution of (1) in $\S 1.5$. Chapter II will be devoted to operators with variable lower-order coefficients. In $\S 2.1$ we shall construct solutions of (1) by successive iteration in the same way as in [20]. In $\S 2.2$ necessary conditions for well-posedness in $\mathscr{E}^{*_{x}}$ will be given by the same arguments as in [11] when the coefficients of $P(x, D)$ are analytic. It will be shown in $\S 2.3$ that the condition (2) is necessary and sufficient for the Cauchy problem (1) to be $\mathscr{E}$-well posed and to have the finite propagation property, using the same arguments as in [12]. In $\S 2.4$ the same results as in $\S 1.4$ will be given for operators with variable lowerorder coefficients. The class of hyperbolicity can be determined explicitly when $n \leqq 3$. In $\S$ A.1 we shall give a procedure to determine the class of hyperbolicity when $n \leqq 3$.

The author would like to thank Professors M. Matsumura and K. Kajitani for many useful discussions.

\section{Chapter I}

\section{Operators with constant coefficients}

\subsection{The Cauchy problem}

In this chapter we assume that $P(D) \equiv P(x, D)$.

Definition 1.1.1. Assume that $P_{m}(\xi)$ is a hyperbolic polynomial with respect to $\vartheta$ and let $\Gamma$ be a cone in $\boldsymbol{R}^{n} \backslash\{0\}$ and $\xi^{0} \in \boldsymbol{R}^{n} \backslash\{0\}$. Then we define $\delta(P ; \Gamma) \equiv \delta(\Gamma)$ and $\delta\left(P ; \xi^{0}\right) \equiv \delta\left(\xi^{0}\right)$ by

$$
\begin{aligned}
& \delta(\Gamma)=\inf \left\{\delta ; \delta \geqq 0 \text { and there exists } \gamma \text { in } \boldsymbol{R} \text { such that } P\left(\xi-i s(1+|\xi|)^{\delta} \vartheta\right) \neq 0\right. \\
& \quad \text { for every } \xi \text { in } \Gamma \text { and } s>\gamma\}, \\
& \delta\left(\xi^{0}\right)=\inf \left\{\delta(\Gamma) ; \Gamma \text { is a conic neighborhood of } \xi^{0}\right\} .
\end{aligned}
$$


Lemma 1.1.2. Let $\Gamma$ be a cone in $R^{n} \backslash\{0\}$ and $\xi^{0} \in R^{n} \backslash\{0\}$. Then $\delta(\Gamma)$ and $\delta\left(\xi^{0}\right)$ have the following properties: (i) $\delta\left(\Gamma_{1}\right) \leqq \delta\left(\Gamma_{2}\right)$ if $\Gamma_{j}, j=1,2$, are cones in $\boldsymbol{R}^{n} \backslash\{0\}$ and $\Gamma_{1} \subset \Gamma_{2}$. (ii) $\delta\left(\xi^{0}\right)=\delta\left(\lambda \xi^{0}\right)$ for $\lambda>0$. (iii) There is a conic neighborhood $\Gamma_{0}$ of $\xi^{0}$ such that $\delta\left(\xi^{0}\right)=\delta\left(\Gamma_{0}\right)$. (iv) There is a conic neighborhood $\Gamma_{0}$ of $\xi^{0}$ such that $\delta(\xi) \leqq \delta\left(\xi^{0}\right)$ for every $\xi \in \Gamma_{0}$. (v) $\delta\left(\xi^{0}\right)$ is a rational number. (vi) If $\delta\left(\xi^{0}\right)$ $>0$, then for every conic neighborhood $\Gamma$ of $\xi^{0}$ there are sequences $\left\{s_{j}\right\} \subset \boldsymbol{R}$ and $\left\{\xi^{j}\right\} \subset \Gamma$ and a positive number $C$ such that $\left|\xi^{j}\right| \rightarrow \infty, s_{j}=C\left|\xi^{j}\right|^{\mid\left(\xi^{0}\right)}(1+o(1))$ as $j \rightarrow \infty$ and $P\left(\xi^{j}-i s_{j} \vartheta\right)=0$.

Proof. (i) and (ii) are obvious. Let us prove the assertion (iii). We may assume that $\left|\hat{\xi}^{0}\right|=1$. Consider the system

$$
\left\{\begin{array}{l}
P(\xi-i s \vartheta)=0, \quad s \in R, \\
|\xi|^{2}=r^{2}, \quad r>0, \\
\left|\xi-r \xi^{0}\right|^{2} \leqq \varepsilon^{2} r^{2}, \quad 0<\varepsilon \leqq \varepsilon_{0} .
\end{array}\right.
$$

Seidenberg's lemma asserts that there are systems $T_{1}, \cdots, T_{N}$ consisting of a finite number of real polynomial equations and inequalities in $(s, r, \varepsilon)$ such that for fixed $(s, r, \varepsilon)$ the existence of real solutions $\xi$ of (1.1.1) is equivalent to the condition that $(s, r, \varepsilon)$ satisfies at least one of the systems $T_{1}, \cdots, T_{N}$ (see, e.g., [6]). Let $\left\{p_{j}\right\}$ be the collection of all polynomials in $(s, r, \varepsilon)$ which are contained in the systems $T_{1}, \cdots, T_{N}$. The polynomial $\prod_{j} p_{j}(s, r, \varepsilon)$ has a factorization

$$
\prod_{j} p_{j}(s, r, \varepsilon)=p^{1}(s, r, \varepsilon)^{j_{1}} \cdots p^{\mu}(s, r, \varepsilon)^{j^{j}} .
$$

Put $p(s, r, \varepsilon)=\prod_{j=1}^{\mu} p^{j}(s, r, \varepsilon)$ and let $\Delta(r, \varepsilon)$ be the discriminant of $p(s, r, \varepsilon)$ with respect to $s$. Since $\Delta(r, \varepsilon) \neq 0$, there are positive numbers $\varepsilon_{1}, r_{1}$ and a positive integer $l$ such that $\varepsilon_{1} \leqq \varepsilon_{0}$ and $\Delta(r, \varepsilon) \neq 0$ for $r^{-1 / l} \leqq \varepsilon \leqq \varepsilon_{1}, r \geqq r_{1}$. Thus $p(s, r, \varepsilon)$ $=0$ has only simple roots for $(r, \varepsilon) \in D \equiv\left\{(r, \varepsilon) \in R^{2} ; r \geqq r_{1}\right.$ and $\left.r^{-1 / l} \leqq \varepsilon \leqq r_{1}\right\}$. Let $\phi_{j}(r, \varepsilon), 1 \leqq j \leqq \omega$, be the roots of $p(s, r, \varepsilon)=0$. Then the $\phi_{j}(r, \varepsilon)$ are analytic in $D$. Moreover $\phi_{j}(r, \varepsilon)$ is real for every $(r, \varepsilon)$ in $D$ if $\phi_{j}\left(r_{2}, \varepsilon_{2}\right)$ is real for some $\left(r_{2}, \varepsilon_{2}\right)$ in $D$, since $p(s, r, \varepsilon)$ is a real polynomial. Thus we can assume that

$$
\begin{aligned}
& \phi_{1}(r, \varepsilon)<\phi_{2}(r, \varepsilon)<\cdots<\phi_{\nu}(r, \varepsilon), \\
& \operatorname{Im} \phi_{j}(r, \varepsilon) \neq 0, \quad \nu+1 \leqq j \leqq \omega, \quad(r, \varepsilon) \in D .
\end{aligned}
$$

If there is $(\xi, s)$ satisfying (1.1.1) for some $\left(r_{2}, \varepsilon_{2}\right)$ in $D$, then there is $(\xi, s)$ satisfying (1.1.1) for each $(r, \varepsilon)$ in $D$. Put

$$
\hat{s} \equiv \hat{s}(r, \varepsilon)=\sup \left\{s ; \xi \in \boldsymbol{R}^{n} \text { and } \xi \text { satisfies (1.1.1) for }(s, r, \varepsilon)\right\} .
$$

Then we have $\hat{s}=\phi_{j_{0}}(r, \varepsilon)$ in $D$ for some $j_{0}, 0 \leqq j_{0} \leqq \nu$, where $\phi_{0}(r, \varepsilon)=-\infty$. Here we have defined $\hat{s}(r, \varepsilon)=-\infty$ unless there is $(\xi, s)$ satisfying (1.1.1) for fixed 
$(r, \varepsilon)$. Write $p(s, r, \varepsilon)=\sum_{j, k} a_{j k}(\varepsilon) s^{j} r^{k}$. Then, modifying $\varepsilon_{1}$, if necessary, we can assume that $a_{j k}(\varepsilon) \neq 0$ for $0<\varepsilon \leqq \varepsilon_{1}$ if $a_{j k}(\varepsilon) \neq 0$. For each $\varepsilon$ with $0<\varepsilon \leqq \varepsilon_{1}$ there are real number $C_{j}(\varepsilon)$ and $\delta_{j} \in Q$ such that

$$
\phi_{j}(r, \varepsilon)=C_{j}(\varepsilon) r^{\delta_{j}}(1+o(1)) \quad \text { as } r \rightarrow \infty,(r, \varepsilon) \in D, 1 \leqq j \leqq \nu .
$$

Here the $C_{j}(\varepsilon)$ and $\delta_{j}$ are determined by the Newton polygon for $p(s, r, \varepsilon)$. Therefore the $\delta_{j}$ and $\operatorname{sgn} C_{j}(\varepsilon)$ are independent of $\varepsilon$. Put $\delta_{0}=0$. Then we have

$$
\delta(\Gamma)= \begin{cases}\max \left(\delta_{j_{0}}, 0\right), & \text { if } C_{j_{0}}\left(\varepsilon_{1}\right)>0 \\ 0, & \text { otherwise }\end{cases}
$$

if $\Gamma$ is a conic neighborhood of $\xi^{0}$ and $\Gamma \subset\left\{\xi \in \boldsymbol{R}^{n} ;|\xi-| \xi\left|\xi^{0}\right| \leqq \varepsilon_{1}|\xi|\right\} \equiv \Gamma_{0}$. This proves (iii). The assertions (iv)-(vi) easily follow from the above. Q.E.D.

LEMma 1.1.3. The set $\left\{\delta(P ; \xi) ; \xi \in \mathbb{R}^{n} \backslash\{0\}\right\}$ is a finite set.

Proof. In (1.1.1) we regard $s, r, \varepsilon$ and $\xi^{0}\left(\left|\xi^{0}\right|=1\right)$ as parameters and repeat the same arguments as in the proof of Lemma 1.1.2. Then we obtain the polynomial $p\left(s, r, \varepsilon, \xi^{0}\right)$ instead of $p(s, r, \varepsilon)$ in the proof of Lemma 1.1.2. For each fixed $\xi^{0}$ with $\left|\xi^{0}\right|=1$ we define $\hat{s} \equiv \hat{s}(r, \varepsilon)$ by (1.1.2). Then we have $p\left(\hat{s}, r, \varepsilon, \xi^{0}\right)=0$ or $\hat{s}=-\infty$. Regarding $p\left(s, r, \varepsilon, \xi^{0}\right)$ as a polynomial of $(s, r)$, the Newton polygon of $p\left(s, r, \varepsilon, \xi^{0}\right)$ depends on whether the coefficient of each monomial contained in $p\left(s, r, \varepsilon, \xi^{0}\right)$ vanishes or not. Thus we have at most finitely many Newton polygons as $\xi^{0}$ varies. From this Lemma 1.1.3 easily follows.

Q.E.D.

Definition 1.1.4. Let $1<\kappa \leqq \infty$. An operator $P(D)$ is called hyperbolic of class $*_{x}$ with respect to $\vartheta$ if there exist a closed cone $K$ in $\boldsymbol{R}^{n}$ and $E(x) \equiv$ $E(P, \vartheta ; x) \in \mathscr{D}^{*_{\kappa^{\prime}}}$ such that $K \backslash\{0\} \subset\left\{x \in \boldsymbol{R}^{n} ; x \cdot \vartheta>0\right\}$ and

$$
P(D) E(x)=\delta(x), \operatorname{supp} E \subset K \text {. }
$$

Then $E(P, \vartheta ; x)$ is called the fundamental solution.

REMARK. The fundamental solution $E(P, \vartheta ; x)$ is unique if it exists.

Proposition 1.1.5 (Gårding [4], Larsson [15]). Let $1<\kappa \leqq \infty . \quad P(D)$ is hyperbolic of class $*_{\kappa}$ with respect to $\vartheta$ if and only if $P_{m}(\xi)$ is hyperbolic with respect to $\vartheta$ and $\kappa \leqq 1 / \delta(P)$ for $*_{\kappa}=(\kappa)$ and $\kappa<1 / \delta(P)$ for $*_{\kappa}=\{\kappa\}$, where

$$
\delta(P)=\max \left\{\delta(P ; \xi) ; \xi \in \boldsymbol{R}^{n} \backslash\{0\}\right\} .
$$




\subsection{Hyperbolic polynomials}

Let $H(\xi)$ be a hyperbolic polynomial with respect to $\vartheta$, homogeneous of degree $m$ and let $S(\xi)$ be a homogeneous polynomial of degree $m^{\prime}, m^{\prime}<m$. For $\xi^{0} \in \boldsymbol{R}^{n} \backslash\{0\}$ we put

$$
\begin{aligned}
N_{\xi^{0}}=\left\{\hat{\gamma}(s) ; \hat{\gamma}(s)=s^{-1}\left(\xi^{0}+\sum_{j=1}^{\infty} s^{j / l} \xi^{j}\right), \xi^{j} \in \boldsymbol{R}^{n}, l \in N \text { and } s \hat{\gamma}(s)\right. \text { is } \\
\\
\text { convergent in a neighborhood of } s=0\} .
\end{aligned}
$$

Definition 1.2.1. Let $\xi^{0} \in \boldsymbol{R}^{n} \backslash\{0\}$. For each $\hat{\eta}(s) \in N_{\hat{\xi}^{0}}$ we define the rational number $n(\hat{\eta}) \equiv n(H, S ; \hat{\eta})$ by

$$
S(\hat{\eta}(s)-i \vartheta) / H(\hat{\eta}(s)-i \vartheta)=c(\hat{\eta}) s^{-n(\hat{\eta})}(1+o(1)) \quad \text { as } s \rightarrow+0,
$$

where $c(\hat{\eta}) \neq 0$. Here we have defined $n(\hat{\eta})=-\infty$ if $S(\hat{\gamma}(s)-i \vartheta) \equiv 0$. Moreover we define

$$
n\left(\xi^{0}\right) \equiv n\left(H, S ; \xi^{0}\right)=\operatorname{supp}\left\{n(\hat{\eta}) ; \hat{\eta} \in N_{\xi^{0}}\right\} .
$$

Definition 1.2.2. Let $\Gamma$ be a cone in $\boldsymbol{R}^{n} \backslash\{0\}$. We define $n(\Gamma) \equiv n(H, S ; \Gamma)$ by

$$
n(\Gamma)=\inf \left\{k ;|S(\xi-i \vartheta) / H(\xi-i \vartheta)| \leqq C\left(1+|\xi|^{k} \text { for every } \xi \in \Gamma\right\}^{\dagger} .\right.
$$

Lemma 1.2.3. Let $\Gamma$ be a cone in $\boldsymbol{R}^{n} \backslash\{0\}$ and $\xi^{0} \in \boldsymbol{R}^{n} \backslash\{0\}$. Then $n(\Gamma)$ and $n\left(\xi^{0}\right)$ have the following properties: (i) $n\left(\Gamma_{1}\right) \leqq n\left(\Gamma_{2}\right)$ if $\Gamma_{j}, j=1,2$, are cones in $\boldsymbol{R}^{n} \backslash\{0\}$ and $\Gamma_{1} \subset \Gamma_{2}$. (ii) $n\left(\xi^{0}\right)=n\left(\lambda \xi^{0}\right)$ for $\lambda \in \boldsymbol{R} \backslash\{0\}$. (iii) $n\left(\xi^{0}\right) \leqq n(\Gamma)$ if $\Gamma$ is a conic neighborhood of $\xi^{0}$. (iv) There is a conic neighborhood $\Gamma_{0}$ of $\xi^{0}$ such that

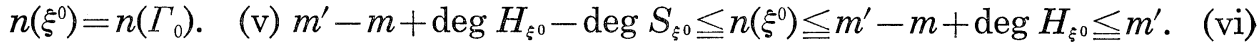
$n\left(\xi^{0}\right)=m^{\prime}-m+\operatorname{deg} H_{\xi^{0}}$ if $S\left(\xi^{0}\right) \neq 0$. (vii) $n\left(\xi^{0}\right)=m^{\prime}-m$ if $H\left(\xi^{0}\right) \neq 0$. (viii) $W e$ assume that $H(\xi)$ and $S(\xi)$ have no non-constant common divisors and that $H(\xi)$ $=\prod_{k} h_{k}(\xi)^{j_{k}}$. Then $n\left(\xi^{0}\right)=m^{\prime}-m+\operatorname{deg} H_{\xi^{0}}$ if $\left(\partial / \partial \xi_{1}\right) R\left(\xi^{0}\right) \neq 0$, where $R(\xi)=$ $\prod_{k} h_{k}(\xi)$. Here $H_{\xi 0}$ is the localization of $H$ at $\xi^{0}$ which is defined by $H\left(s^{-1} \xi^{0}+\xi\right)$ $=s^{r}\left(H_{\xi^{0}}(\xi)+o(1)\right)$ as $s \rightarrow 0, H_{\xi^{0}}(\xi) \neq 0$.

Proof. The assertions (i)-(iii) are obvious. Let us prove the assertion (iv). We may assume that $\left|\xi^{0}\right|=1$ and that $S(\xi) \neq 0$. Consider the system

$$
\left\{\begin{array}{l}
\phi|H(\xi-i \vartheta)|^{2}-|S(\xi-i \vartheta)|^{2}=0, \quad \phi \in R, \\
|\xi|^{2}=r^{2}, \quad r>0, \\
\left|\xi-r \xi^{0}\right|^{2} \leqq \varepsilon^{2} r^{2}, \quad 0<\varepsilon \leqq \varepsilon_{0} .
\end{array}\right.
$$

We repeat the same arguments as in the proof of Lemma 1.1.2. Put

$$
\hat{\phi} \equiv \hat{\phi}(r, \varepsilon)=\max \left\{\phi ; \xi \in \boldsymbol{R}^{n} \text { and } \xi \text { satisfies (1.2.2) for }(\phi, r, \varepsilon)\right\}
$$

\footnotetext{
$\dagger$ Here and in the sequel $C$ denotes a positive constant.
} 
for each fixed $(r, \varepsilon)$ and

$$
D=\left\{(r, \varepsilon) \in R^{2} ; r \geqq r_{1} \text { and } r^{-1 / l} \leqq \varepsilon \leqq \varepsilon_{1}\right\}
$$

for sufficiently small $\varepsilon_{1}$ and sufficiently large $r_{1}>0$ and $l \in N$. Then there is a polynomial $p(\phi, r, \varepsilon)$ such that $\hat{\phi}(r, \varepsilon)$ is a simple root of $p(\phi, r, \varepsilon)=0$ if $(r, \varepsilon)$ $\in D$. We can write $\hat{\phi}(r, \varepsilon)$ in the form

$$
\hat{\phi}(r, \varepsilon)=C(\varepsilon) r^{\rho}+r^{\rho} \psi(r, \varepsilon), \psi(r, \varepsilon)=o(1) \quad \text { as } r \rightarrow \infty, \text { for }(r, \varepsilon) \in D,
$$

where $C(\varepsilon) \neq 0$ and $\rho$ are determined by the Newton polygon of $p(\phi, r, \varepsilon)$ with respect to $(\phi, r)$, since $\hat{\phi}(r, \varepsilon)>0$. Modifying $\varepsilon_{1}$, if necessary, $\rho$ can be taken to be independent of $\varepsilon$ in (1.2.3) and $C(\varepsilon)$ is represented as a Puiseux series of ย. (1.2.3) implies that

$$
n\left(\Gamma_{0}\right)=\inf \left\{n(\Gamma) ; \Gamma \text { is a conic neighborhood of } \xi^{0}\right\}=\rho / 2,
$$

where $\Gamma_{0}=\left\{\xi \in \boldsymbol{R}^{n} ;|\xi-| \xi\left|\xi^{0}\right| \leqq \varepsilon_{1}|\xi|\right\}$. Let us first prove that there are a positive numbers $\nu$ and $C$ such that

$$
|\psi(r, \varepsilon)| \leqq C r^{-\nu} \quad \text { in } D .
$$

Assume that $\psi(r, \varepsilon) \neq 0$. Put

$$
q(\psi, r, \varepsilon)=r^{-\mu} p\left(C(\varepsilon) r^{\rho}+r^{\rho} \psi, r, \varepsilon\right)
$$

where $p\left(C(\varepsilon) r^{\rho}+r^{\rho} \psi, r, \varepsilon\right)=O\left(r^{\mu}\right)$ as $r \rightarrow \infty$. Then $q(\psi, r, \varepsilon)$ is a polynomial of $\psi$ and $r^{-1 / L}$ and its coefficients are Puiseux series of $\varepsilon$, where $L \in N$ is chosen suitably. We note that a Puiseux series $a(\varepsilon)$ of $\varepsilon$ does not vanish for $0<\varepsilon \leqq \varepsilon_{1}$ if $a(\varepsilon) \neq 0$ and $\varepsilon_{1}$ is sufficiently small. Thus we can write $\psi(r, \varepsilon)$ in the form

$$
\psi(r, \varepsilon)=C_{1}(\varepsilon) r^{-\nu^{\prime}}(1+o(1)) \quad \text { as } r \rightarrow \infty \text { for }(r, \varepsilon) \in D,
$$

where $C_{1}(\varepsilon) \neq 0$ and $\nu^{\prime}>0$ are determined by the Newton polygon of $q(\psi, r, \varepsilon)$ with respect to $\left(\psi, r^{-1 / L}\right)$. Modifying $\varepsilon_{1}$, if necessary, $\nu^{\prime}$ can be chosen to be independent of $\varepsilon$ in (1.2.5). Put $\hat{\psi}(r, \varepsilon)=r^{\nu^{\prime}} \psi(r, \varepsilon)$. Then we have

$$
q_{1}(\hat{\psi}, \varepsilon)+r^{-\nu_{1}} q_{2}(\hat{\psi}, r, \varepsilon)=0
$$

where $\nu_{1}>0, q_{1}(\psi, \varepsilon)$ is a polynomial of $\psi, q_{2}(\psi, r, \varepsilon)$ is a polynomial of $\psi$ and $r^{-1 / L}$ and the coefficients of $q_{1}(\psi, \varepsilon)$ and $q_{2}(\psi, r, \varepsilon)$ are Puiseux series of $\varepsilon$. There are positive numbers $k, \varepsilon_{1}$ and $C$ and $k_{1} \in R$ such that

$$
\left|q_{1}(\psi, \varepsilon)\right| \geqq C \varepsilon^{-k_{1}} \quad \text { if }|\psi| \geqq \varepsilon^{-k} \text { and } 0<\varepsilon \leqq \varepsilon_{1} .
$$

On the other hand we have

$$
\left|q_{2}(\psi, r, \varepsilon)\right| \leqq C \varepsilon^{-k_{2}}, \quad r \geqq r_{1}, \quad 0<\varepsilon \leqq \varepsilon_{1},
$$


for some constants $C$ and $k_{2}$ if $|\psi| \geqq \varepsilon^{-k}$. Thus $\left|q_{1}(\psi, \varepsilon)\right|>r^{-\nu_{1}}\left|q_{2}(\psi, r, \varepsilon)\right|$ if $|\psi|$ $\geqq \varepsilon^{-k}, r \geqq r_{1}, r^{-1 / l} \leqq \varepsilon \leqq \varepsilon_{1}$ and $l \in N$ is sufficiently large. This implies that $|\hat{\psi}(r, \varepsilon)| \leqq r^{k / l}$ for $(r, \varepsilon) \in D$. Modifying $l$, if necessary, we have (1.2.4) with $\nu=\nu^{\prime}-k / l>0$. Since $C(\varepsilon)=A \varepsilon^{a}(1+o(1)), \varepsilon \rightarrow+0$, for some $A \in R \backslash\{0\}$ and $a \in \boldsymbol{Q}$, it follows from (1.2.4) that

$$
\hat{\phi}\left(r, r^{-1 / l}\right)=A r^{\rho-a / l}(1+o(1)) \quad \text { as } r \rightarrow \infty,
$$

modifying $l$. Now assume that

$$
n\left(\xi^{0}\right)<n\left(\Gamma_{0}\right)=\rho / 2 .
$$

Then we can assume that $n\left(\xi^{0}\right)<(\rho-a / l) / 2$, modifying $l$. Thus there are integers $p$ and $q$ such that $p>0, p$ and $q$ are relatively prime and

$$
2 n\left(\xi^{0}\right)<q / p<\rho-a / l .
$$

Let $l^{\prime}$ be the least common multiple of $p$ and $l$. Following the proof of Lemm 1.2 in [19], we consider the system

$$
\left\{\begin{array}{l}
s r^{q^{\prime}}|H(\xi-i \vartheta)|^{2}-|S(\xi-i \vartheta)|^{2}=0, \\
|\xi|^{2}=r^{2 l^{\prime}}, \quad r \geqq 1, \\
\left|\xi-r^{l^{\prime}} \xi^{0}\right|^{2} \leqq r^{2 l^{\prime}-l^{\prime \prime}},
\end{array}\right.
$$

where $q^{\prime}=l^{\prime} q / p, l^{\prime \prime}=2 l^{\prime} / l$. Then Seidenberg's lemma asserts for every $j, 1 \leqq$ $j \leqq n$, the existence of a condition $H_{j}$ consisting of a finite number of systems of real polynomial equations and inequalities in $\left(\xi_{1}, \cdots, \xi_{j}, s\right)$ such that for every $\left(\xi_{1}, \cdots, \xi_{j}, s\right) \in \boldsymbol{R}^{j+1}$ the following conditions are equivalent:

(i) There exist real $\xi_{j+1}, \cdots, \xi_{n}, r$ so that $(\xi, s, r)$ satisfies the system (1.2.8).

(ii) At least one of the systems in $H_{j}$ is satisfied by $\left(\xi_{1}, \cdots, \xi_{j}, s\right)$.

Assume that for some $j, 1 \leqq j \leqq n$, there are Puiseux series $\gamma_{1}(s), \cdots, \gamma_{j-1}(s)$, convergent and real for all large real $s$, such that the system (1.2.8) is satisfied by some real $\xi=\left(\gamma_{1}(s), \cdots, \gamma_{j-1}(s), \xi_{j}, \cdots, \xi_{n}\right)$ and $r$ for some arbitrarily large real $s$. It follows from (1.2.6) and (1.2.7) that there are real $\xi$ and $r$ satisfying (1.2.8) for some arbitrarily large real $s$. This means that the assumption is valid when $j=1$. If $\left(\gamma_{1}(s), \cdots, \gamma_{j-1}(s), \xi_{j}, s\right)$ satisfies the condition $H_{j}$, then there is a Puiseux series $\gamma_{j}(s)$, convergent and real for all large real $s$, such that $\left(\gamma_{1}(s), \cdots, \gamma_{j-1}(s), \gamma_{j}(s), s\right)$ satisfies the condition $H_{j}$ (see [19]). Thus we obtain Puiseux series $\gamma_{1}(s), \cdots, \gamma_{n}(s)$ such that $\xi=\left(\gamma_{1}(s), \cdots, \gamma_{n}(s)\right)$ and $r=|\xi|^{1 / l^{\prime}}$ satisfy the system (1.2.8) for all sufficiently large real $s$. Put $\eta(s)=\left(\gamma_{1}(s), \cdots\right.$, $\left.\gamma_{n}(s)\right)$. We can write $\eta(s)$ in the form

$$
\eta(s)=C s^{l^{\prime} h / L}\left(\xi^{0}+\sum_{j=1}^{\infty} s^{-j / L} \xi^{j}\right),
$$


where $C>0$ and $h, L \in N$. In fact, we have $r(s)=C^{\prime} s^{h / L}(1+o(1))$ ] as $s \rightarrow \infty$, where $r(s)=|\eta(s)|^{1 / l^{\prime}}$. Replacing $C s^{l^{\prime} h / L}$ by $s^{-1}$, we put

$$
\hat{\eta}(s)=s^{-1}\left(\xi^{0}+\sum_{j=1}^{\infty} s^{j / L^{\prime}} \tilde{\xi}^{j}\right),
$$

where $L^{\prime}=l^{\prime} h, \tilde{\xi}^{j}=C^{j / L^{\prime}} \xi^{j}$. Then we have

$$
|S(\hat{\gamma}(s)-i \vartheta) / H(\hat{\gamma}(s)-i \vartheta)|^{2}=C s^{-L / L^{\prime}-q / p}(1+o(1)), \quad \text { as } s \rightarrow+0 .
$$

This implies that $2 n\left(\xi^{0}\right) \geqq q / p+L / L^{\prime}$, which contradicts (1.2.7). This completes the proof of the assertion (iv). If $\hat{\gamma}(s)=s^{-1} \xi^{0}+\xi^{1}$ and $S_{\xi^{0}}\left(\xi^{1}-i \vartheta\right) \neq 0$, we have $n(\hat{\eta}(s)) \geqq m^{\prime}-m+\operatorname{deg} H_{\xi^{0}}-\operatorname{deg} S_{\xi^{0}}$, which implies that $n\left(\xi^{0}\right) \geqq m^{\prime}-m+\operatorname{deg} H_{\xi_{0}}$ $-\operatorname{deg} S_{\xi^{0}}$. On the other hand we have

$$
|H(\hat{\eta}(s)-i \vartheta)| \geqq C s^{a}, \quad 0<s \leqq s_{0}, \quad a=-m+\operatorname{deg} H_{\xi 0},
$$

for $\hat{\eta} \in N_{\xi^{0}}$ and some $s_{0}>0$, In fact, we have

$$
|H(\hat{\eta}(s)-i \vartheta)| \geqq C \tilde{H}(\hat{\gamma}(s)) \geqq C\left|H^{(\alpha)}(\hat{\eta}(s))\right|
$$

(see [7]). Moreover $H^{(\alpha)}\left(\xi^{0}\right) \neq 0$ if $\alpha=\left(\operatorname{deg} H_{\xi_{0}}, 0, \cdots, 0\right)$ (see [1]). This proves the assertion (v). The assertion (vi) easily follows from (v). The assertion (vii) is obvious in virtue of (iv). Finally let us prove the assertion (viii). Assume that $H\left(\xi^{0}\right)=0$. For every $\varepsilon>0$ there exists $\xi_{s} \in \boldsymbol{R}^{n}$ such that $H\left(\xi_{s}\right)=0$, $S\left(\xi_{\varepsilon}\right) \neq 0$ and $\left|\xi^{0}-\xi_{s}\right|<\varepsilon$, since $H(\xi)$ and $S(\xi)$ are relatively prime. Moreover the assumption of (viii) implies that $\operatorname{deg} H_{\xi^{0}}=\operatorname{deg} H_{\xi_{\varepsilon}}$ if $\varepsilon$ is sufficiently small. Since

$$
\left|S\left(s^{-1} \xi_{s}-i \vartheta\right) / H\left(s^{-1} \xi_{s}-i \vartheta\right)\right| \geqq C s^{a}, \quad 0<s \leqq s_{0}, \quad a=-m^{\prime}+m-\operatorname{deg} H_{\xi_{s}},
$$

we have $n\left(\xi^{0}\right) \geqq m^{\prime}-m+\operatorname{deg} H_{\xi^{0}}$, which proves the assertion (viii). In fact, (viii) is an immediate consequence of (vii) if $H\left(\xi^{0}\right) \neq 0$.

Q.E.D.

LeMma 1.2.4. Let $\xi^{0} \in \boldsymbol{R}^{n} \backslash\{0\}$ and $\hat{\eta}(s) \in N_{\xi^{0}}$ and put

$$
\hat{\eta}_{a}^{\kappa}(s)=s^{\kappa /(1-\kappa)} \hat{\eta}\left(s^{1 /(1-\kappa)}\right)+a \vartheta \quad \text { for } 0 \leqq \kappa<1 \text { and } a \in \boldsymbol{R} .
$$

Then we have

$$
\left(\hat{\eta}_{a}^{\kappa}\right)_{a^{\prime}}^{\kappa^{\prime}}(s)=\hat{\eta}_{j a}^{k+k^{\prime}-\kappa \kappa^{\prime}}(s)+a s^{\kappa^{\prime} /\left(1-\kappa^{\prime}\right)} \vartheta, \quad 0 \leqq \kappa, \kappa^{\prime}<1 \quad \text { and } \quad a, a^{\prime} \in \boldsymbol{R} .
$$

Moreover there exist a rational number $\kappa_{0}$ with $0 \leqq \kappa_{0}<1$ and $a_{0} \in \boldsymbol{R}$ such that

$$
n\left(H, S ; \hat{\eta}_{a_{0}}^{\kappa_{0}}\right) \geqq n\left(H, S ; \hat{\eta}_{a}^{\kappa}\right), \quad 0 \leqq \kappa<1, \quad a \in \boldsymbol{R},
$$

where $n\left(H, S ; \hat{\eta}_{a}^{\kappa}\right)$ is defined by (1.2.1) even if $\hat{\eta}_{a}^{\kappa} \notin N_{\xi^{0}}$.

Proof. (1.2.9) easily follows from the definition of $\hat{\eta}_{a}^{k}(s)$. Let $\tau_{j}(s), 1 \leqq$ 
$j \leqq m$, and $\lambda_{k}(s), 1 \leqq k \leqq t$, be the roots of $H(s \hat{\eta}(s)+\tau \vartheta)=0$ and $S(s \hat{\eta}(s)+\tau \vartheta)=0$, respectively. Write $S(s \hat{\eta}(s)+\tau \vartheta)=\sum_{j=0}^{t} q_{j}(s) \tau^{t-j}$. Then we have

$$
\begin{aligned}
H(\hat{\eta}(s)+\tau \vartheta) & =s^{-m} H(\vartheta) \prod_{k=1}^{m}\left(s \tau-\tau_{k}(s)\right), \\
S(\hat{\eta}(s)+\tau \vartheta) & =s^{-m^{\prime}} q_{0}(s) \prod_{k=1}^{t}\left(s \tau-\lambda_{k}(s)\right) .
\end{aligned}
$$

Put $\mu_{k}=\operatorname{order} \tau_{k}(s), \mu_{k}^{\prime}=\operatorname{order} \lambda_{k}(s)$ and $\alpha=\operatorname{order} q_{0}(s)$, where $\mu=\operatorname{order} \lambda(s)$ implies that $\lambda(s)=A s^{\mu}(1+o(1))$ as $s \rightarrow+0$ for some non-zero constant $A$ if $\mu<$ $\infty$ and that $\lambda(s) \equiv 0$ if $\mu=\infty$. Let $0 \leqq \kappa<1$. It is obvious that $\alpha=$ order $q(s)$ $\geqq 0$. If $\alpha=\infty$, then $n\left(H, S ; \hat{r}_{a}^{k}\right)=-\infty$ for all $a \in \boldsymbol{R}$ and $0 \leqq \kappa<1$. So let us consider the case $\alpha<\infty$. We have

$$
\begin{aligned}
& S\left(\hat{\gamma}(s)+s^{-\kappa}(\tau-i) \vartheta\right) / H\left(\hat{\gamma}(s)+s^{-\kappa}(\tau-i) \vartheta\right)=s^{a}\left(d_{k}(\tau)+o(1)\right) \quad \text { as } s \rightarrow+0, \\
& a=m-m^{\prime}+\alpha-\sum_{k=1}^{m} \min \left(\mu_{k}, 1-\kappa\right)+\sum_{k=1}^{t} \min \left(\mu_{k}^{\prime}, 1-\kappa\right), \\
& s^{-\kappa\left(m^{\prime}-m\right)} S\left(s^{k} \hat{\gamma}(s)+(\tau-i) \vartheta\right) / H\left(s^{k} \hat{\gamma}(s)+(\tau-i) \vartheta\right)=s^{a}\left(d_{\tau, \kappa}+o(1)\right)
\end{aligned}
$$

$$
a=-\kappa\left(m^{\prime}-m\right)-n\left(H, S ; \hat{\gamma}_{\tau}^{\kappa}\right)(1-\kappa),
$$

where $d_{\kappa}(\tau)$ is a rational function of $\tau$ and $d_{\tau, \kappa}$ is a non-zero constant depending on $\tau$ and $\kappa$. Since the $\tau_{k}(s)$ are real for $s>0$, it follows that $\left|d_{k}(\tau)\right|<\infty$ for real $\tau$. (1.2.10) and (1.2.11) imply that

$$
\begin{aligned}
& n\left(H, S ; \hat{\eta}_{a}^{\kappa}\right)=m^{\prime}-m+(1-\kappa)^{-1}\left\{-\alpha+\sum_{k=1}^{m} \min \left(\mu_{k}, 1-\kappa\right)\right. \\
&\left.-\sum_{k=1}^{t} \min \left(\mu_{k}^{\prime}, 1-\kappa\right)\right\} \equiv n(\kappa) \quad \text { if } d_{\kappa}(a) \neq 0,
\end{aligned}
$$

and that

$$
n\left(H, S ; \hat{\eta}_{a}^{\kappa}\right)<n(\kappa) \quad \text { if } d_{\kappa}(a)=0 .
$$

Put $\beta=\min _{0 \leqq j \leqq t}$ order $q_{j}(s)$. Then we have $\mu_{k} \geqq 0,1 \leqq k \leqq m$, and $\sum_{\mu_{k}^{\prime} \leqq 0} \mu_{k}^{\prime}=\beta-\alpha$ (see Lemma A.1.2). The set $\left\{\mu_{k} ; 0<\mu_{k} \leqq 1\right\}$ is written as follows:

$$
\left\{l_{k} ; k=1, \cdots, N\right\}=\left\{\mu_{k} ; 0<\mu_{k} \leqq 1\right\}, \quad 0<l_{1}<l_{2}<\cdots<l_{N} \leqq 1 .
$$

Now assume that $\kappa_{k+1} \equiv 1-l_{k+1}<\kappa<\kappa_{k} \equiv 1-l_{k}, 0 \leqq k \leqq N$, where $l_{0}=0$ and $l_{N+1}$ $=1$. Then we have

$$
\begin{aligned}
n\left(\kappa_{k+1}\right)-n(\kappa)= & \left(l_{k+1}^{-1}-(1-\kappa)^{-1}\right)\left\{-\beta+\sum_{\mu_{j} \leqq l_{k}} \mu_{j}-\sum_{0<\mu_{j}^{\prime} \leqq 1-\kappa} \mu_{j}^{\prime}\right\} \\
& +\sum_{1-\kappa<\mu^{\prime} \leqq l_{k+1}}\left(1-\mu_{j}^{\prime} / l_{k+1}\right), \quad 0 \leqq k \leqq N, \\
n\left(\kappa_{k}\right)-n(\kappa)= & \left(l_{k}^{-1}-(1-\kappa)^{-1}\right)\left\{-\beta+\sum_{\mu_{j} \leqq l_{k}} \mu_{j}-\sum_{0<\mu_{j}^{\prime} \leqq 1-\kappa} \mu_{j}^{\prime}\right\} \\
& +\sum_{l_{k}<\mu_{j}^{\prime} \leqq 1-\kappa}\left(\mu_{j}^{\prime} / l_{k}-1\right), \quad 1 \leqq k \leqq N .
\end{aligned}
$$

Since $\beta \geqq 0$, we have $n\left(\kappa_{1}\right) \geqq n(\kappa)$ for $\kappa_{1}<\kappa<1$. Moreover we have, for $\kappa_{k+1}<\kappa$ $<\kappa_{k}$, 


$$
\begin{array}{ll}
n\left(\kappa_{k}\right) \geqq n(\kappa), & \text { if }-\beta+\sum_{\mu_{j} \leqq l_{k}} \mu_{j}-\sum_{0<\mu_{j}^{\prime} \leqq 1-\kappa} \mu_{j}^{\prime} \geqq 0, \\
n\left(\kappa_{k+1}\right)>n(\kappa), & \text { otherwise. }
\end{array}
$$

Therefore we have

$$
\max _{1 \leqq k \leqq N+1} n\left(\kappa_{k}\right)=\sup _{0 \leqq \kappa<1} n(\kappa) .
$$

From (1.2.12) and (1.2.13) Lemma 1.2.4 easily follows.

Q.E.D.

TheOREm 1.2.5. Assume that $P_{m}(\xi)$ is hyperbolic with respect to $\vartheta$ and let $\xi^{0} \in \boldsymbol{R}^{n} \backslash\{0\}$. Then we have

$$
\begin{aligned}
\max & \left(\delta\left(P ; \xi^{0}\right), \delta\left(P ;-\xi^{0}\right)\right) \\
& =\max _{0 \leqq j \leqq m-1} n_{+}\left(P_{m}, Q_{j} ; \xi^{0}\right) /\left(m-j+n_{+}\left(P_{m}, Q_{j} ; \xi^{0}\right)\right) \\
& \left(\leqq \max \left(\left(\operatorname{deg} P_{m \xi^{0}}-1\right) / \operatorname{deg} P_{m \xi^{0}}, 0\right)\right),
\end{aligned}
$$

where $n_{+}\left(H, S ; \xi^{0}\right)=\max \left(n\left(H, S ; \xi^{0}\right), 0\right)$. In particular,

$$
\delta(P)=\max _{|\xi|=1,0 \leqq j \leqq m-1} n_{+}\left(P_{m}, Q_{j} ; \xi\right) /\left(m-j+n_{+}\left(P_{m}, Q_{j} ; \xi\right)\right) .
$$

REMARK. (i) Theorem 1.2.5 is an extension of Svensson's result ([19]). In fact, it follows from Proposition 1.1.5 that $P(D)$ is hyperbolic of class $(\kappa)$ with respect to $\vartheta$ if and only if $P_{m}(\xi)$ is hyperbolic with respect to $\vartheta$ and

$$
\left|Q_{j}(\xi-i \vartheta) / P_{m}(\xi-i \vartheta)\right| \leqq C(1+|\xi|)^{(m-j) /(k-1)}, \quad \xi \in \boldsymbol{R}^{n}, \quad 0 \leqq j \leqq m-1 .
$$

(ii) The left-hand side of (1.2.14) can not be replaced by $\delta\left(P ; \xi^{0}\right)$. For example, if $P(\xi)=\xi_{1}^{2}-\xi_{2}$ and $\xi^{0}=(0,1)$, then $\delta\left(P ; \xi^{0}\right)=0$ and $n\left(P_{2}, Q_{1} ; \xi^{0}\right)=1$.

Proof. Let us first prove that

$$
\delta\left(P ; \xi^{0}\right) \leqq \max _{0 \leqq j \leqq m-1} n_{+}\left(P_{m}, Q_{j} ; \xi^{0}\right) /\left(m-j+n_{+}\left(P_{m}, Q_{j} ; \xi^{0}\right)\right) \equiv \delta .
$$

Let $\Gamma_{0}$ be a sufficiently small conic neighborhood of $\xi^{0}$. Then it follows from Lemma 1.2.3 that

$$
\begin{aligned}
& \left|P_{m}\left(\xi-i \gamma(1+|\xi|)^{\delta} \vartheta\right)\right|-\left|\sum_{j=0}^{m-1} Q_{j}\left(\xi-i \gamma(1+|\xi|)^{\delta} \vartheta\right)\right| \\
& \geqq\left|P_{m}\left(\xi-i \gamma(1+|\xi|)^{\delta} \vartheta\right)\right|\left(1-\sum_{j=0}^{m-1} C \gamma^{j-m}\right. \\
& \left.\quad \times(1+|\xi|)^{\delta\left(j-m-n_{+}\left(P_{m}, Q_{j} ; \xi 0\right)\right)+n_{+}\left(P_{m}, Q_{j} ; \xi^{0}\right)}\right)>0
\end{aligned}
$$

if $\xi \in \Gamma_{0}$ and $\gamma$ is sufficiently large, since $\delta\left(j-m-n_{+}\left(P_{m}, Q_{j} ; \xi^{0}\right)\right)+n_{+}\left(P_{m}, Q_{j} ; \xi^{0}\right)$ $\leqq 0,0 \leqq j \leqq m-1$. This proves (1.2.15). We may assume that $\delta>0$, that is, there is $j$ with $0 \leqq j \leqq m-1$ such that $n\left(P_{m}, Q_{j} ; \xi^{0}\right)>0$. In fact, Theorem 1.2.5 easily follows from (1.2.15) if $\delta=0$. Then for every $\varepsilon, 0<\varepsilon<\delta$, there is $\hat{\eta}(s)$ in $N_{\xi 0}$ such that

$$
0<\delta-\varepsilon<\max _{0 \leqq j \leqq m-1} n_{+}\left(P_{m}, Q_{j} ; \hat{\eta}\right) /\left(m-j+n_{+}\left(P_{m}, Q_{j} ; \hat{\eta}\right)\right) \equiv \delta(\hat{\eta}) \leqq \delta<1 .
$$


By Lemma 1.2.4 we can assume without loss of generality that

$$
\begin{aligned}
& n\left(P_{m}, Q_{j} ; \hat{\eta}\right) \geqq n\left(P_{m}, Q_{j} ; \hat{\eta}_{a}^{\kappa}\right) \\
& \text { if } n_{+}\left(P_{m}, Q_{j} ; \hat{\eta}\right) /\left(m-j+n_{+}\left(P_{m}, Q_{j} ; \hat{\eta}\right)\right)=\delta(\hat{\eta}), \quad 0 \leqq \kappa<1 \quad \text { and } \quad a \in \boldsymbol{R} .
\end{aligned}
$$

Moreover we can assume that

$$
n\left(P_{m}, Q_{j} ; \hat{\eta}\right) \geqq n\left(P_{m}, Q_{j} ; \hat{\eta}_{a}^{0}\right) \quad \text { for all } a \in R \quad \text { and } \quad 0 \leqq j \leqq m-1 .
$$

Write

$$
\begin{aligned}
P_{m}(\hat{\eta}(s)+\tau \vartheta) & =s^{\rho}\left(a_{m} \tau^{k_{m}}+b_{m}(\tau)\right)+o\left(s^{\rho}\right) \quad \text { as } s \rightarrow+0, \\
Q_{j}(\hat{\eta}(s)+\tau \vartheta) & =s^{\rho-n\left(P_{m}, Q_{j} ; \hat{\eta}\right)}\left(a_{j} \tau^{k_{j}}+b_{j}(\tau)\right)+o\left(s^{\rho-n\left(P_{m}, Q_{j} ; \hat{\eta}\right)}\right) \quad \text { as } s \rightarrow+0,
\end{aligned}
$$

where $a_{j} \neq 0$ and the $b_{j}(\tau)$ are polynomials of degree less than $k_{j}$ if $j=m$ or $n\left(P_{m}, Q_{j} ; \hat{\eta}\right)>-\infty$. Put

$$
\begin{aligned}
& p \equiv p(\hat{\eta})=\delta(\hat{\eta}) /(1-\delta(\hat{\eta}))(>0), \\
& I(\tau ; s, \gamma)=\gamma^{m} s^{m p} P\left(\gamma^{-1} s^{-p}(\hat{\eta}(s)+\tau \vartheta)\right) .
\end{aligned}
$$

Then we have

$$
\begin{aligned}
I(\tau ; s, \gamma)= & s^{\rho}\left\{a_{m} \tau^{k_{m}}+b_{m}(\tau)+\sum_{p(m-j)=n\left(P_{m}, Q_{i ;} ; \hat{\eta}\right)} \gamma^{m-j}\left(a_{j} \tau^{k_{j}}+b_{j}(\tau)\right)\right\} \\
& +o\left(s^{\rho}\right) \quad \text { as } s \rightarrow+0 .
\end{aligned}
$$

In fact, $p(m-j)-n\left(P_{m}, Q_{j} ; \hat{\eta}\right) \geqq 0$ for $0 \leqq j \leqq m-1$. Now let us prove that

$$
\begin{aligned}
& k_{j} \leqq k_{m}-(1+p)(m-j) \\
& \text { if } p(m-j)=n\left(P_{m}, Q_{j} ; \hat{\eta}\right) \text { and } 0 \leqq j \leqq m-1 .
\end{aligned}
$$

For sufficiently small $\kappa>0$ we have

$$
\begin{array}{rr}
P_{m}\left(\hat{\eta}(s)+s^{-\kappa} \tau \vartheta\right)=s^{-m_{x}} P_{m}\left(s^{\kappa} \hat{\eta}(s)+\tau \vartheta\right)=s^{\rho-k_{m} x}\left(a_{m} \tau^{k_{m}}+o(1)\right) & \text { as } s \rightarrow+0 \\
Q_{j}\left(\hat{\eta}(s)+s^{-\kappa} \tau \vartheta\right)=s^{-j \kappa} Q_{j}\left(s^{\kappa} \hat{\gamma}(s)+\tau \vartheta\right)=s^{\rho-n\left(P_{m}, Q_{j} ; \hat{\eta}\right)-k_{j} \kappa}\left(a_{j} \tau^{k_{j}}+o(1)\right) & \text { as } s \rightarrow+0,
\end{array}
$$

for each fixed $\tau \in \boldsymbol{R}$. Thus it follows from (1.2.18) and (1.2.19) that

$$
\begin{aligned}
& m-j+n\left(P_{m}, Q_{j} ; \hat{\eta}\right) \leqq k_{m}-k_{j} \\
& \text { if } p(m-j)=n\left(P_{m}, Q_{j} ; \hat{\eta}\right) \text { and } 0 \leqq j \leqq m-1 .
\end{aligned}
$$

This proves (1.2.17). Now put

$$
I^{0}(\tau, \gamma)=a_{m} \tau^{k_{m}}+b_{m}(\tau)+\sum_{p(m-j)=n\left(P_{m}, Q_{j} ; \hat{\eta}\right)} \gamma^{m-j}\left(a_{j} \tau^{k_{j}}+b_{j}(\tau)\right) .
$$

Since $0<(m-j) /\left(k_{m}-k_{j}\right) \leqq 1 /(1+p)<1$ for $p(m-j)=n\left(P_{m}, Q_{j} ; \hat{\eta}\right)$, it follows 
from the Newton polygon of $I^{0}(\tau ; \gamma)$ that there is a root $\tau^{0}(\gamma)$ of $I^{0}(\tau ; \gamma)=0$ such that $\tau^{0}(\gamma)=A \gamma^{\nu}(1+o(1))$ as $|\gamma| \rightarrow \infty$ for some rational number $\nu$ with $0<\nu<1$ and some non-zero constant $A$. Thus $I^{0}\left(\tau, \gamma_{0}\right)$ has a root $\tau_{0}$ such that $\gamma_{0} \operatorname{Im} \tau_{0}$ $<0$, if $\gamma_{0}$ in $R$ is suitably chosen. Then $I\left(\tau ; s, \gamma_{0}\right)=0$ has a root $\tau(s)$ such that $\tau(s)=\tau_{0}(1+o(1))$ as $s \rightarrow+0$. Hence we have

$$
\begin{aligned}
P\left( \pm t^{-1}\left(\xi^{0}+\sum_{j=1}^{\infty}\left(\left|\gamma_{0}\right|^{-1} t\right)^{j /((p+1) l)} \xi^{j}\right) \pm t^{-1}\left(\left|\gamma_{0}\right|^{-1} t\right)^{1 /(p+1)} \tau\left(\left(\left|\gamma_{0}\right|^{-1} t\right)^{1 /(p+1)}\right) \vartheta\right) & =0 \\
\text { if } \gamma_{0} & = \pm\left|\gamma_{0}\right| .
\end{aligned}
$$

This means that

$$
\max \left(\delta\left(\xi^{0}\right), \delta\left(-\xi^{0}\right)\right) \geqq p /(p+1)=\delta(\hat{\eta})>\delta-\varepsilon .
$$

The proof is complete.

Q.E.D.

From now on we assume that $P_{m}(\xi)$ is a hyperbolic polynomial with respect to $\vartheta$ and denote by $\Gamma\left(P_{m}, \vartheta\right)$ the connected component of the set $\left\{\xi \in \boldsymbol{R}^{n} ; P_{m}(\xi) \neq 0\right\}$ which contains $\vartheta$.

Lemma 1.2.6. Let $M$ be a compact set in $\Gamma\left(P_{m}, \vartheta\right)$. Then there exists a positive number $\gamma_{M}$ such that

$$
P\left(\xi-i \gamma(1+|\xi|)^{\delta(P)} \eta\right) \neq 0 \quad \text { for } \gamma>\gamma_{M} \quad \text { and } \eta \in M .
$$

Proof. From hyperbolicity of $P_{m}$ and Lemma 4.1.1 in [7] it follows that

$$
\begin{array}{r}
\left|P_{m}\left(\xi-i \gamma(1+|\xi|)^{\hat{\delta}(P)} \eta\right)\right| \geqq C \gamma^{m}(1+|\xi|)^{m \hat{o}(P)} \tilde{P}_{m}\left(\gamma^{-1}(1+|\xi|)^{-\hat{o}(P)} \xi\right), \\
\xi \in \boldsymbol{R}^{n} \text { and } \eta \in M .
\end{array}
$$

Moreover we have

$$
\begin{array}{r}
\left|Q_{j}\left(\xi-i \gamma(1+|\xi|)^{\delta(P)} \eta\right)\right| \leqq C \gamma^{j}(1+|\xi|)^{j \delta(P)} \tilde{Q}_{j}\left(\gamma^{-1}(1+|\xi|)^{-\delta(P)} \xi\right), \\
\xi \in \boldsymbol{R}^{n} \text { and } \eta \in M, \quad 0 \leqq j \leqq m-1 .
\end{array}
$$

On the other hand we have

$$
\tilde{Q}_{j}(\xi) / \tilde{P}_{m}(\xi) \leqq C(1+|\xi|)^{n_{+}\left(P_{m}, Q_{j}\right)}, \quad \xi \in \boldsymbol{R}^{n}, \quad 0 \leqq j \leqq m-1,
$$

where $n_{+}\left(P_{m}, Q_{j}\right)=\max _{|\xi|=1} n_{+}\left(P_{m}, Q_{j} ; \xi\right)$. In fact, it follows from Lemma 3.1.5 in [7] that

$$
\tilde{Q}_{j}(\xi) \leqq C^{\prime} \tilde{Q}_{j}(\xi-i \vartheta) \leqq C(1+|\xi|)^{n_{+}\left(P_{m}, Q_{j}\right)} \tilde{P}_{m}(\xi), \quad \xi \in \boldsymbol{R}^{n}, \quad 0 \leqq j \leqq m-1 .
$$

By (1.2.20)-(1.2.22) we have

$$
\begin{aligned}
\tilde{P}_{m}\left(\gamma^{-1}(1+|\xi|)^{-\delta(P)} \xi\right)^{-1}\{\mid & P_{m}\left(\xi-i \gamma(1+|\xi|)^{\delta(P)} \eta\right) \mid \\
& \left.-\sum_{j=0}^{m-1}\left|Q_{j}\left(\xi-i \gamma(1+|\xi|)^{\delta(P)} \eta\right)\right|\right\} \geqq C \gamma^{m}(1+|\xi|)^{m \delta(P)}(1-1 / \gamma), \\
& \xi \in \boldsymbol{R}^{n} \text { and } \eta \in M, \quad \gamma \geqq 1,
\end{aligned}
$$


which proves the lemma.

Q.E.D.

Lemma 1.2.7. Let $\xi^{0} \in R^{n} \backslash\{0\}$ and let $M$ be a compact set in $\Gamma\left(P_{m \xi^{0}}, \vartheta\right)$ and $\max _{0 \leqq j \leqq m-1} n_{+}\left(P_{m}, Q_{j} ; \xi^{0}\right) /\left(m-j+n_{+}\left(P_{m}, Q_{j} ; \xi^{0}\right)\right) \leqq \delta<1$. Then there exist $a$ conic neighborhood $\Gamma$ of $\xi^{0}$ and positive numbers $t_{0}, \gamma_{0}$ and $C$ such that

$$
\begin{array}{ll}
P\left(\xi-i \gamma_{0}(1+|\xi|)^{\delta} \vartheta-i t|\xi| \eta\right) \neq 0 \quad \text { for } \xi \in \Gamma \text { with } \\
\\
\quad|\xi| \geqq C, \quad \eta \in M \text { and } 0 \leqq t \leqq t_{0} .
\end{array}
$$

Proof. If $\Gamma$ is a sufficiently small conic neighborhood of $\xi^{0}$, there is $\gamma_{0}$ $>0$ such that

$$
P\left(\xi-i \gamma(1+|\xi|)^{\delta} \vartheta\right) \neq 0 \quad \text { for } \xi \in \Gamma \text { and } \gamma \geqq \gamma_{0}-1 \text {. }
$$

Put

$$
f(\nu, r, \zeta, t, \eta)=P\left(r \nu^{-1} \xi^{0}+r \zeta-i \gamma_{0}\left(r \nu^{-1}\right)^{\delta} \vartheta-i t r \nu^{-\delta} \eta\right)
$$

when $\zeta \in R^{n},|\zeta| \leqq \varepsilon, \eta \in M, 0<\nu \leqq \nu_{0}, r \geqq r_{0},|t| \leqq t_{0}$ and $\operatorname{Re} t \geqq 0$. Then we have

$$
\begin{aligned}
& f(\nu, r, \zeta, t, \eta)=r^{m} \nu^{a}\left\{P_{m \xi^{0}}\left(\nu^{\delta} \zeta-i \gamma_{0} r^{\delta-1} \vartheta-i t \eta\right)+o(1)\right\} \\
& a=-m+(1-\delta) \operatorname{deg} P_{m \xi 0 .} \\
& \text { as } r \rightarrow \infty \text { and } \nu \rightarrow+0,
\end{aligned}
$$

In fact,

$$
\begin{aligned}
& Q_{j}\left(r \nu^{-1} \xi^{0}+r \zeta-i \gamma_{0}\left(r \nu^{-1}\right)^{\delta} \vartheta-i t r \nu^{-\delta} \eta\right) \\
& \quad=r^{j} \nu^{-j \delta} Q_{j}\left(\nu^{-1+\delta} \xi^{0}+\nu^{\delta} \zeta-i \gamma_{0} r^{\delta-1} \vartheta-i t \eta\right) \\
& \quad=r^{j} \nu^{a}\left\{Q_{j \xi}\left(\nu^{\delta} \zeta-i \gamma_{0} r^{\delta-1} \vartheta-i t \eta\right)+o(1)\right\} \quad \text { as } \nu \rightarrow+0, \quad 0 \leqq j \leqq m-1,
\end{aligned}
$$

From the assertion (v) of Lemma 1.2.3 it follows that $m-j-\left(\operatorname{deg} P_{m \xi^{0}}-\right.$ $\left.\operatorname{deg} Q_{j \xi 0}\right)(1-\delta) \geqq 0$. Thus (1.2.24) follows from (1.2.25). (1.2.23) implies that

$$
\begin{aligned}
f(\nu, r, \zeta, s, t, \eta) \neq 0 \quad \text { when }|t| \leqq t_{0}, \quad \operatorname{Re} t=0, \quad \zeta \in R^{n}, \\
|\zeta| \leqq \varepsilon, \quad \eta \in M, \quad 0<\nu \leqq \nu_{0} \quad \text { and } \quad r \geqq r_{0},
\end{aligned}
$$

with a modification of $\nu_{0}$ and $t_{0}$, if necessary. Moreover we have

$$
\begin{aligned}
& f(\nu, r, \zeta, s, t, \eta) \neq 0 \quad \text { when }|t|=t_{0}, \operatorname{Re} t \geqq 0, \quad \zeta \in R^{n}, \\
&|\zeta| \leqq \varepsilon, \quad \eta \in M, \quad 0<\nu \leqq \nu_{0} \quad \text { and } \quad r \geqq r_{0},
\end{aligned}
$$

modifying $\nu_{0}$ and $r_{0}$, if necessary. In fact, from (1.2.24) it follows that 


$$
\begin{aligned}
& \begin{array}{l}
f(\nu, r, \zeta, t, \eta)=r^{m} \nu^{-m+(1-\delta) a}\left\{t^{a} P_{m \xi^{0}}\left(\nu^{\delta} t^{-1} \zeta-i \gamma_{0} r^{\delta-1} t^{-1} \vartheta-i \eta\right)+o(1)\right\} \\
\text { as } r \rightarrow \infty
\end{array} \\
& a=\operatorname{deg} P_{m \xi^{0} .}
\end{aligned}
$$

Since $P_{m \xi 0}(-i \eta) \neq 0, \eta \in M$, we have (1.2.26). Now assume that there are $\eta^{1} \in$ $M, \zeta^{1} \in R^{n}$ with $\left|\zeta^{1}\right| \leqq \varepsilon, t_{1} \in C$ with $\left|t_{1}\right|<t_{0}$ and $\operatorname{Re} t_{1}>0, r_{1} \geqq r_{0}$ and $\nu_{1}$ with $0<\nu_{1}$ $\leqq \nu_{0}$ such that $f\left(\nu_{1}, r_{1}, \zeta^{1}, t_{1}, \eta^{1}\right)=0$. We may assume that $M$ is convex and $\vartheta \in$ $M$. Denote by $t(\theta)$ a root of $f\left(\nu_{1}, r_{1}, \zeta^{1}, t,(1-\theta) \eta^{1}+\theta \vartheta\right)=0$ such that $t(0)=t_{1}$ and $t(\theta)$ is continuous. Then we can define $t(\theta)$ for $\theta \in[0,1]$ since $\operatorname{Re} t(\theta)>0$ and $|t(\theta)|<t_{0}$. Thus we have $f\left(\nu_{1}, r_{1}, \zeta^{1}, t(1), \vartheta\right)=0$, which is a contradiction to (1.2.23). This proves the lemma.

Q.E.D.

\subsection{Generalized wave front sets}

Let us first state the Paley-Wiener theorem for $\mathscr{D}^{*_{x}}$ and $\mathscr{E}^{*_{\kappa^{\prime}}}$ (see, e.g., [13], [14]).

Proposition 1.3.1. Let $K$ be a compact convex set in $\boldsymbol{R}^{n}$ and $1<\kappa<\infty$. (i) An entire function $\hat{\phi}(\zeta)$ on $C^{n}$ is the Fourier-Laplace transform of $\phi \in \mathscr{D}_{K}^{(k)}$ (resp. $\mathscr{D}_{K}^{[k\}}$ ) if and only if for every $L>0$ there is a positive number $C$ (resp. there are positive numbers $L$ and $C$ ) such that

$$
|\hat{\phi}(\zeta)| \leqq C \exp \left[-L|\zeta|^{1 / x}+h_{K}(\operatorname{Im} \zeta)\right], \quad \zeta \in C^{n},
$$

where $h_{K}(\eta)=\sup _{x \in K} x \cdot \eta$. (ii) The following conditions are equivalent for an entire function $\hat{f}(\zeta)$ on $\boldsymbol{C}^{n}$ : (a) $\hat{f}(\zeta)$ is the Fourier-Laplace transform of $f \in \mathscr{D}_{K}^{(\kappa)}$ (resp. $\left.\mathscr{D}_{K}^{\{r\}^{\prime}}\right)$, where $\mathscr{D}_{K}^{*_{\kappa^{\prime}}}=\left\{f \in \mathscr{D}^{*_{\kappa^{\prime}}} ; \operatorname{supp} f \subset K\right\}$. (b) There are constants $L$ and $C$ (resp. for every $L>0$ there is a constant $C$ ) such that

$$
|\hat{f}(\zeta)| \leqq C \exp \left[L|\zeta|^{1 / \kappa}+h_{K}(\operatorname{Im} \zeta)\right], \quad \zeta \in C^{n} .
$$

(c) There are constants $L$ and $C$ (resp. for every $L>0$ there is a constant $C$ ) such that

$$
|\hat{f}(\xi)| \leqq C \exp \left[L|\xi|^{1 / i}\right], \quad \xi \in \boldsymbol{R}^{n}
$$

and for every $\varepsilon>0$ there is a constant $C_{\varepsilon}$ such that

$$
|\hat{f}(\zeta)| \leqq C_{\varepsilon} \exp \left[h_{K}(\operatorname{Im} \zeta)+\varepsilon|\zeta|\right], \quad \zeta \in C^{n} .
$$

Let $\kappa_{1}>1$ and $f \in \mathscr{D}^{\left(\kappa_{1}\right)^{\prime}}(\Omega)$, where $\Omega$ is an open set in $\boldsymbol{R}^{n}$. Proposition 1.3.1 leads naturally to the following definition.

Definition 1.3.2. Let $\kappa_{1} \leqq \kappa<\infty$. (i) $W F_{(k)}(f)\left(\right.$ resp. $\left.W F_{\{k\}}(f)\right)$ is defined as the complement in $T^{*}(\Omega) \backslash 0$ of the collection of all $\left(x^{0}, \xi^{0}\right)$ in $T^{*}(\Omega) \backslash 0$ such 
that there are a neighborhood $U$ of $x^{0}$ in $\Omega$ and a conic neighborhood $\Gamma$ of $\xi^{0}$ such that for every $\phi \in \mathscr{D}^{\left(\kappa_{1}\right)}(U)$ and every $L>0$ there is a positive number $C$ (resp. for every $\phi \in \mathscr{D}^{\left({ }^{(}\right)}(U)$ there are positive numbers $L$ and $C$ ) satisfying

$$
|\mathscr{F}[\phi f](\xi)| \leqq C \exp \left[-L|\xi|^{1 / x}\right], \quad \xi \in \Gamma,
$$

where $\mathscr{F}[f](\xi) \equiv \hat{f}(\xi)$ denotes the Fourier transform of $f$ (see [8]). (ii) $W F^{(\kappa)}(f)$ (resp. $W F^{\{x\}}(f)$ ) is defined as the complement in $T^{*}(\Omega) \backslash 0$ of the collection of all $\left(x^{0}, \xi^{0}\right)$ in $T^{*}(\Omega) \backslash 0$ such that there are a neighborhood $U$ of $x^{0}$ in $\Omega$ and a conic neighborhood $\Gamma$ of $\xi^{0}$ such that for every $\phi \in \mathscr{D}^{\left(\kappa_{1}\right)}(U)$ there are constants $L$ and $C$ (resp. for every $\phi \in \mathscr{D}^{\left(\kappa_{1}\right)}(U)$ and every $L>0$ there is a constant $C$ ) satisfying

$$
|\mathscr{F}[\phi f](\xi)| \leqq C \exp \left(L|\xi|^{1 / x}\right), \quad \xi \in \Gamma .
$$

(iii) Moreover we define $W F_{(\infty)}(f)=W F_{\{\infty\}}(f)=W F(f)$ (resp. $W F^{(\infty)}(f)=$ $\left.W F^{\{\infty\}}(f)\right)$ as the complement in $T^{*}(\Omega) \backslash 0$ of the collection of all $\left(x^{0}, \xi^{0}\right)$ in $T^{*}(\Omega) \backslash 0$ such that there are a neighborhood $U$ of $x^{0}$ in $\Omega$ and a conic neighborhood $\Gamma$ of $\xi^{0}$ such that for every $\phi \in \mathscr{D}^{\left(\kappa_{1}\right)}(U)$ and every non-negative integer $N$ there is a positive number $C$ (resp. for every $\phi \in \mathscr{D}^{\left({ }^{\left(n_{1}\right)}\right.}(U)$ there are an integer $N$ and $C>0$ ) satisfying

$$
|\mathscr{F}[\phi f](\xi)| \leqq C(1+|\xi|)^{-N}, \quad \xi \in \Gamma
$$

(see [9]).

The following lemmas give some properties of $W F_{*_{x}}(f)$ and $W F^{*_{r}}(f)$. The proofs of these lemmas can be given in the same way as in [9], [2].

LEMMA 1.3.3. Let $\pi$ be the projection $(x, \xi) \mapsto x$ from $T^{*}(\Omega) \backslash 0$ onto $\Omega$. Then we have

$$
\begin{aligned}
\pi\left(W F_{*_{x}}(f)\right)= & \text { sing supp }{ }_{*_{x}} f \equiv \Omega \backslash\{x \in \Omega ; \text { there is a neighborhood } U \\
& \text { of } \left.x \text { such that }\left.f\right|_{U} \in \mathscr{E}^{*_{\star}}(U)\right\}, \\
\pi\left(W F^{*_{\star}}(f)\right)= & \text { sing supp }{ }^{*_{\star}} f \equiv \Omega \backslash\{x \in \Omega ; \text { there is a neighborhood } U \\
& \text { of } \left.x \text { such that }\left.f\right|_{U} \in \mathscr{D}^{*_{\kappa^{\prime}}}(U)\right\} .
\end{aligned}
$$

Lemma 1.3.4. Let $\phi \in \mathscr{E}^{\left(\kappa_{1}\right)}(\Omega)$. Then we have

$$
W F_{*_{\kappa}}(\phi f) \subset W F_{*_{s}}(f), \quad W F^{*_{\kappa}}(\phi f) \subset W F^{*_{\kappa}}(f) .
$$

Let $\Omega_{j}, j=1,2$, be open sets in $\boldsymbol{R}^{n_{j}}$.

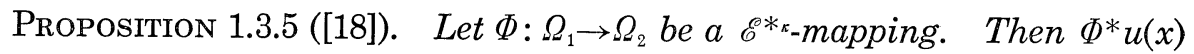
$\equiv u(\Phi(x))$ belongs to $\mathscr{E}^{*}\left(\Omega_{1}\right)$ if $u \in \mathscr{E}^{*} *_{\kappa}\left(\Omega_{2}\right)$. 
Lemma 1.3.6. Let $\left(x^{0}, \xi^{0}\right) \in T^{*}(\Omega) \backslash 0$ and $\kappa_{1} \leqq \kappa<\infty$. (i) $\left(x^{0}, \xi^{0}\right)$ does not belong to $W F_{(x)}(f)$ (resp. $\left.W F_{\{k\}}(f)\right)$ if and only if there are a conic neighborhood $\Gamma$ of $\xi^{0}$ and $\phi \in \mathscr{D}^{\left({ }^{\left(x_{1}\right)}\right.}(\Omega)$ such that $\phi\left(x^{0}\right) \neq 0$ and for every $L>0$ there is a positive number $C$ (resp. there are positive numbers $L$ and $C$ ) satisfying

$$
|\mathscr{F}[\phi f](\xi)| \leqq C \exp \left[-L|\xi|^{1 / \kappa}\right], \quad \xi \in \Gamma .
$$

(ii) $\left(x^{0}, \xi^{0}\right)$ does not belong to $W F^{(k)}(f)\left(\right.$ resp. $\left.W F^{\{k\}}(f)\right)$ if and only if there are a conic neighborhood $\Gamma$ of $\xi^{0}$ and $\phi \in \mathscr{D}^{\left(x_{1}\right)}(\Omega)$ such that $\phi\left(x^{0}\right) \neq 0$ and there are constants $L$ and $C$ (resp. for every $L>0$ there is a constant $C$ ) satisfying

$$
|\mathscr{F}[\phi f](\xi)| \leqq C \exp \left[L|\xi|^{1 / x}\right], \quad \xi \in \Gamma .
$$

Deginition 1.3.7. Let $\Gamma$ be a closed cone in $T^{*}(\Omega) \backslash 0$ and $1<\kappa$. Define

$$
\mathscr{D}_{\Gamma}^{(\kappa)}(\Omega)=\left\{u \in \mathscr{D}^{(\kappa)^{\prime}}(\Omega) ; W F(u) \subset \Gamma\right\} .
$$

We introduce the topology in $\mathscr{D}_{\Gamma}^{(k)^{\prime}}(\Omega)$ by the semi norms of the weak topology in $\mathscr{D}^{(s)}(\Omega)$ together with the semi norms $u \mapsto \sup _{\xi \in r,|\xi| \geqq 1}|\mathscr{F}[\phi u](\xi)||\xi|^{N}$, where $K$ is a compact set in $\Omega, \gamma$ is a closed cone, $K \times \gamma \cap \Gamma=\emptyset, \phi \in \mathscr{D}^{(x)}$ with supp $\phi$ $\subset K$ and $N=0,1,2, \cdots$.

REMARK. $\quad \mathscr{E}^{\left(\kappa^{\prime}\right)}(\Omega)$ is dense in $\mathscr{D}_{\Gamma}^{(k)}(\Omega)$.

Lemma 1.3.8. Let $\Phi: \Omega_{1} \rightarrow \Omega_{2}$ be a real analytic mapping. Define $N_{\Phi}=$ $\left\{(\Phi(y), \xi) \in T^{*}\left(\Omega_{2}\right) \backslash 0 ;{ }^{t} D \Phi_{y} \xi=0\right\}$. Let $\Gamma$ be a closed cone in $T^{*}\left(\Omega_{2}\right) \backslash 0$ such that $\Gamma \cap N_{\Phi}=\emptyset$. Then the pull-back $\Phi^{*}: C^{\infty}\left(\Omega_{2}\right) \rightarrow C^{\infty}\left(\Omega_{1}\right)$ has a unique continuous extension: $\mathscr{D}_{\Gamma}^{(\kappa)}\left(\Omega_{2}\right) \rightarrow \mathscr{D}^{(\kappa)^{\prime}}\left(\Omega_{1}\right)$. Let $f \in \mathscr{D}^{\left(\kappa_{1}\right)^{\prime}}\left(\Omega_{2}\right)$ and $\kappa_{1} \leqq \kappa . \quad$ If $W F_{*_{k}}(f) \cap N_{\Phi}=\emptyset$, we have

$$
W F_{*_{\varepsilon}}\left(\Phi^{*} f\right) \subset \Phi^{*} W F_{*_{x}}(f) \equiv\left\{\left(y,{ }^{t} D \Phi_{y} \xi\right) ;(\Phi(y), \xi) \in W F_{*_{\varepsilon}}(f)\right\} .
$$

If $W F(f) \cap N_{\Phi}=\emptyset$, we have

$$
W F^{*_{\kappa}}\left(\Phi^{*} f\right) \subset \Phi^{*} W F^{*_{\kappa}}(f) .
$$

REMARK. $W F_{*_{x}}(f)$ and $W F^{*_{r}}(f)$ are invariant under real analytic coordinate transformations.

Lemma 1.3.9. Let $\Phi: \Omega_{1} \rightarrow \Omega_{2}$ be a real analytic proper mapping. The push-forward $\Phi_{*}={ }^{t}\left(\Phi^{*}\right): \mathscr{D}^{* \kappa_{1^{\prime}}}\left(\Omega_{1}\right) \rightarrow \mathscr{D}^{*_{k_{1}}}\left(\Omega_{2}\right)$ can be defined by

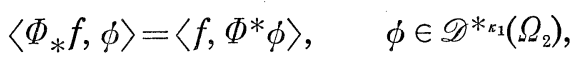

for $f \in \mathscr{D}^{*} \kappa_{1^{\prime}}\left(\Omega_{1}\right)$. Then we have

$$
\begin{aligned}
& W F_{*_{x}}\left(\Phi_{*} f\right) \subset\left\{(x, \xi) \in T^{*}\left(\Omega_{2}\right) \backslash 0 ; x=\Phi(y),\left(y,{ }^{t} D \Phi_{y} \xi\right) \in W F_{*_{x}}(f)\right. \\
& \text { for some } \left.y \in \Omega_{1} \text { or } x=\Phi(y),{ }^{t} D \Phi_{y} \xi=0 \text { for some } y \in \Omega_{1}\right\},
\end{aligned}
$$




$$
\begin{aligned}
& W F^{*_{k}}\left(\Phi_{*} f\right) \subset\left\{(x, \xi) \in T^{*}\left(\Omega_{2}\right) \backslash 0 ; x=\Phi(y),\left(y,{ }^{t} D \Phi_{y} \xi\right) \in W F^{*_{k}}(f)\right. \\
& \left.\quad \text { for some } y \in \Omega_{1} \text { or } x=\Phi(y),{ }^{t} D \Phi_{y} \xi=0 \text { for some } y \in \Omega_{1}\right\} .
\end{aligned}
$$

LEMma 1.3.10. Let $u_{j} \in \mathscr{D}^{*_{\kappa_{1}}}\left(\Omega_{j}\right), j=1,2$, and $1<\kappa_{1} \leqq \kappa$. Then we have

$$
\begin{aligned}
& W F_{*_{x}}\left(u_{1} \otimes u_{2}\right) \subset \bigcap_{\kappa_{1} \leqq s \leqq s}\left\{W F^{* s}\left(u_{1}\right) \times W F_{*_{x_{1}}}\left(u_{2}\right) \cup W F_{*_{\kappa_{1}}}\left(u_{1}\right) \times W F^{*_{s}}\left(u_{2}\right)\right. \\
& \cup W F_{*_{s}}\left(u_{1}\right) \times W F_{*_{s}}\left(u_{2}\right) \cap \operatorname{supp}_{0}^{* s} u_{1} \times W F_{*_{1}}\left(u_{2}\right) \\
& \cup \operatorname{supp}_{0} u_{1} \times W F_{*_{s}}\left(u_{2}\right) \cap W F_{*_{1}}\left(u_{1}\right) \times \operatorname{supp}_{0}^{* s} u_{2} \\
& \left.\cup W F_{* s}\left(u_{1}\right) \times \operatorname{supp}_{0} u_{2}\right\} \text {, } \\
& W F^{*_{\kappa}}\left(u_{1} \otimes u_{2}\right) \subset \bigcap_{\kappa_{1} \leqq s \leqq \kappa}\left\{W F^{*_{s}}\left(u_{1}\right) \times W F_{*_{\kappa_{1}}}\left(u_{2}\right) \cup W F^{*_{\kappa}}\left(u_{1}\right) \times W F_{*_{s}}\left(u_{2}\right)\right. \\
& \cup W F_{*_{1}}\left(u_{1}\right) \times W F^{*_{s}}\left(u_{2}\right) \cup W F_{*_{s}}\left(u_{1}\right) \times W F^{*_{s}}\left(u_{2}\right) \\
& \cup W F^{*_{k}}\left(u_{1}\right) \times \operatorname{supp}_{0} u_{2} \cup W F_{*_{1}}\left(u_{1}\right) \times \operatorname{supp}_{0}^{* s} u_{2} \\
& \cup W F_{*_{s}}\left(u_{1}\right) \times \operatorname{supp}_{0}^{*_{\kappa}} u_{2} \cup \operatorname{supp}_{0} u_{1} \times W F^{*_{\kappa}}\left(u_{2}\right) \\
& \left.\cup \operatorname{supp}_{0}^{*_{s}} u_{1} \times W F_{*_{1}}\left(u_{2}\right) \cup \operatorname{supp}_{0}^{*_{k}} u_{1} \times W F_{*_{s}}\left(u_{2}\right)\right\} \text {, }
\end{aligned}
$$

where $\operatorname{supp}_{0} u=\{(x, 0) ; x \in \operatorname{supp} u\}$ and $\operatorname{supp}_{0}^{*_{k}} u=\left\{(x, 0) ; x \in \operatorname{sing} \operatorname{supp}^{*_{k}} u\right\}$. Here we have identified $T^{*}\left(\Omega_{1} \times \Omega_{2}\right)$ with $T^{*}\left(\Omega_{1}\right) \times T^{*}\left(\Omega_{2}\right)$.

Let $\pi$ be the projection: $(x, y) \mapsto x$ from $\Omega_{1} \times \Omega_{2}$ onto $\Omega_{1}$ and $\Delta$ be a mapping: $(x, y) \mapsto(x, y, y)$ from $\Omega_{1} \times \Omega_{2}$ into $\Omega_{1} \times \Omega_{2} \times \Omega_{2}$. Moreover let $K(x, y) \in \mathscr{D}^{* \kappa_{x^{\prime}}}\left(\Omega_{1}\right.$ $\left.\times \Omega_{2}\right)$ and $u \in \mathscr{E}^{*} *_{1_{1}}\left(\Omega_{2}\right)$. We define formally

$$
K u=\pi_{*} \circ \Delta^{*}(K \otimes u) .
$$

If $u \in \mathscr{D}^{*_{k_{1}}}$, then we have

$$
\langle K u, \phi\rangle=\langle K(x, y), \phi(x) u(y)\rangle, \quad \phi \in \mathscr{D}^{*_{n_{1}}} .
$$

Put

$W F_{*_{\kappa_{1} \Omega_{2}}}^{\prime}(K)=\left\{(y, \eta) \in T^{*}\left(\Omega_{2}\right) \backslash 0 ;((x, y),(0,-\eta)) \in W F_{*_{k_{1}}}(K)\right.$ for some $\left.x \in \Omega_{1}\right\}$.

LEMmA 1.3.11. Assume that $W F_{*_{k_{1} \Omega_{2}}}^{\prime}(K) \cap W F_{*_{1}}(u)=\emptyset$. Then $K u$ is well-

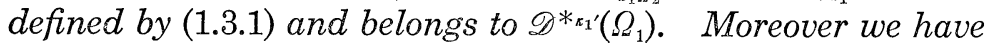

$$
\begin{aligned}
& W F_{*_{k}}(K u) \subset \bigcap_{\kappa_{1} \leqq s \leqq n}\left\{W F^{*_{s^{\prime}}}(K) \circ W F_{*_{\kappa_{1}}}(u) \cup W F_{*_{\kappa_{1}}}^{\prime}(K) \circ W F^{*_{s}}(u)\right. \\
& \cup W F_{*_{s}}^{\prime}(K) \circ W F_{*_{s}}(u) \cup W F_{*_{k_{1}}}^{\prime}(K) \circ \operatorname{supp}_{0}^{* s} u \\
& \left.\cup W F_{*_{s}}^{\prime}(K) \circ \operatorname{supp}_{0} u\right\} \text {, } \\
& W F^{*_{r}}(K u) \subset \bigcap_{\kappa_{1} \leqq s \leqq n}\left\{W F^{*^{\prime}}(K) \circ W F_{*_{\kappa_{1}}}(u) \cup W F^{*_{n^{\prime}}}(K) \circ W F_{*_{s}}(u)\right. \\
& \cup W F_{*_{k_{1}}}^{\prime}(K) \circ W F^{*_{s}}(u) \cup W F_{*_{s}}^{\prime}(K) \circ W F^{*_{s}}(u) \\
& \cup W F^{*_{n^{\prime}}}(K) \circ \operatorname{supp}_{0} u \cup W F_{*_{k_{1}}}^{\prime}(K) \circ \operatorname{supp}_{0}^{* s} u \\
& \left.\cup W F_{* s}^{\prime}(K) \circ \operatorname{supp}_{0}^{* \pi} u\right\} \text {, }
\end{aligned}
$$


where $W^{\prime}=\left\{((x, \xi),(y, \eta)) \in T^{*}\left(\Omega_{1}\right) \times T^{*}\left(\Omega_{2}\right) ;((x, y),(\xi,-\eta)) \in W\right\}$ for $W \subset T^{*}\left(\Omega_{1}\right.$ $\left.\times \Omega_{2}\right)$.

\subsection{Fundamental solutions and singularities of solutions}

From now on we assume that $P_{m}(\xi)$ is hyperbolic with respect to $\vartheta$. The fundamental solution $E(x) \equiv E(P, \vartheta ; x)$ can be represented as

$$
E(P, \vartheta ; x)=(2 \pi)^{-n} \int e^{i x \cdot \zeta} P(\zeta)^{-1} d \zeta,
$$

where $\zeta=\xi-i \gamma(1+|\xi|)^{\delta(P)} \vartheta, \xi \in \boldsymbol{R}^{n}, \gamma>\gamma_{0}$ and $\gamma_{0}$ is sufficiently large. (1.4.1) is interpreted as follows:

$$
\begin{gathered}
\langle E(x), \phi(x)\rangle=(2 \pi)^{-n} \int_{\xi \in \boldsymbol{R}^{n}, \zeta=\xi-i_{\gamma}(1+|\xi|)^{\delta(P)} \vartheta} \hat{\phi}(-\zeta) P(\zeta)^{-1} d \zeta, \\
\gamma>\gamma_{0}, \quad \phi \in \mathscr{D}^{\left(\kappa_{0}\right)},
\end{gathered}
$$

where $\kappa_{0}=1 / \delta(P)$. In fact, it follows from Proposition 1.3.1 that (1.4.2) is meaningful and that $E(x) \in \mathscr{D}^{\left(x_{0}\right)^{\prime}}$. Moreover by Lemma 1.2.6 it is easy to see that ch [supp $E(x)]=\Gamma\left(P_{m}, \vartheta\right)^{*}$, where $\Gamma\left(P_{m}, \vartheta\right)^{*}$ is the dual cone of $\Gamma\left(P_{m}, \vartheta\right)$, that is,

$$
\Gamma\left(P_{m}, \vartheta\right)^{*}=\left\{x \in \boldsymbol{R}^{n} ; x \cdot \eta \geqq 0 \text { for all } \eta \in \Gamma\left(P_{m}, \vartheta\right)\right\},
$$

and $\operatorname{ch}[M]$ denotes the convex hull of $M$, which proves the sufficiency of Proposition 1.1.5. The necessity of proposition 1.1 .5 can be proved in the same way as in [1] (see [15]).

Let $\kappa_{1}>1$ and $x^{0} \in \boldsymbol{R}^{n}$ and let $U$ and $U_{1}$ be neighborhoods of $x^{0}$ and $U_{1} \Subset U$. Then there exist a sequence $\left\{\chi_{N}\right\}_{N=1,2, \ldots}$ in $\mathscr{D}^{\left(\kappa_{1}\right)}(U)$ and a positive number $C$ such that for every $h>0$ there exists a positive number $C_{h}$ satisfying

$$
\left|D^{\alpha+\beta} \chi_{N}(x)\right| \leqq C_{h}(C N)^{|\alpha|} h^{|\beta|}(|\beta| !)^{\alpha_{1}}, \quad|\alpha| \leqq N,
$$

and $\chi_{N}(x)=1$ on $U_{1}$ (see [8]).

DEFinition 1.4.1. Let $f \in \mathscr{D}^{\left(\kappa_{1}\right)^{\prime}}(\Omega)$. Then the analytic wave front set $W F_{A}(f)$ of $f$ is defined as the complement in $T^{*}(\Omega) \backslash 0$ of the collection of all $\left(x^{0}, \xi^{0}\right)$ in $T^{*}(\Omega) \backslash 0$ such that for some sequence $\left\{\chi_{N}\right\}$ of the above type (1.4.3), where $U$ is a sufficiently small neighborhood of $x^{0}$ in $\Omega$, there are a conic neighborhood $\Gamma$ of $\xi^{0}$ and a positive number $C$ satisfying

$$
\left|\mathscr{F}\left[\chi_{N} f\right](\xi)\right| \leqq C(C N)^{N}(1+|\xi|)^{-N}, \quad \xi \in \Gamma, \quad N=1,2, \cdots .
$$

Theorem 1.4.2. Assume that $\left(x^{0}, \xi^{0}\right) \in T^{*}\left(\boldsymbol{R}^{n}\right) \backslash 0$ and $x^{0} \oplus \Gamma\left(P_{m \xi 0}, \vartheta\right)^{*}$. Then we have $\left(x^{0}, \xi^{0}\right) \oplus W F_{A}(E)$, that is, 


$$
W F_{A}(E) \subset \bigcup_{\xi \in \boldsymbol{R}^{n} \backslash\{0\}} \Gamma\left(P_{m \xi}, \vartheta\right)^{*} \times\{\xi\} .
$$

Proof. By the assumption there are $\eta \in \Gamma\left(P_{m \xi^{0}}, \vartheta\right)$ and a neighborhood $U$ of $x^{0}$ such that $x \cdot \eta<0$ for $x \in U$. Let $M$ be a compact convex set in $\Gamma\left(P_{m \xi 0}, \vartheta\right)$ which contains $\eta$ and $\vartheta$ and let $\Gamma, t_{0}$ and $\gamma_{0}$ be as described in Lemma 1.2.7 with $\delta=\delta(P)$. We put

$$
v_{t}(\xi)=\gamma_{0}(1+|\xi|)^{\delta(P)} \vartheta-t|\xi| \Phi(\xi) \eta,
$$

where $\Phi(\xi) \in C^{\infty}\left(\boldsymbol{R}^{n}\right)$ is positively homogeneous of degree 0 in $|\xi| \geqq 1$ and $\Phi(\xi)$ $=1$ on a neighborhood of $\Gamma_{1} \cap\{|\xi| \geqq 1\}$, supp $\Phi(\xi) \subset \Gamma, 0 \leqq \Phi(\xi) \leqq 1$, and $\Gamma_{1}(\subset \Gamma)$ is a conic neighborhood of $\xi^{0}$. Let $\left\{\chi_{N}\right\} \subset \mathscr{D}^{\left({ }^{(}\right)}(U)$ be a sequence which satisfies (1.4.3). It is easy to see that for every $B>0$ there are a positive numbers $C_{B}, A$ and $c$ such that

$$
\begin{aligned}
\left|\hat{\chi}_{N}\left(-\xi+i v_{t}(\xi)+\hat{\xi}\right)\right| \leqq & C_{B}(C N)^{N}\left(1+|\xi-\hat{\xi}|+\left|v_{t}(\xi)\right|\right)^{-N} \\
& \times \exp \left[-B|\xi-\hat{\xi}|^{1 / \alpha_{0}}+A(1+|\xi|)^{\hat{\delta}(P)}-c t|\xi| \Phi(\xi)\right], \\
& \xi \text { and } \hat{\xi} \in \boldsymbol{R}^{n}, \quad N=1,2, \cdots
\end{aligned}
$$

Seidenberg's lemma and Lemmas 1.2.6 and 1.2.7 imply that

$$
\left|P\left(\xi-i v_{t}(\xi)\right)\right|^{-1} \leqq C(1+|\xi|)^{a}, \quad \xi \in \boldsymbol{R}^{n}, \quad 0 \leqq t \leqq t_{0}
$$

for some constants $C$ and $a$. Let $\Gamma_{2}\left(\Subset \Gamma_{1}\right)$ be a conic neighborhood of $\xi_{0}$. Then we have

$$
|\xi-\hat{\xi}| \geqq \varepsilon(|\xi|+|\hat{\xi}|) \quad \text { for some } \varepsilon>0 \quad \text { if } \xi \notin \Gamma_{1} \quad \text { and } \quad \hat{\xi} \in \Gamma_{2} .
$$

On the other hand we have

$$
\begin{aligned}
& \exp \left[-c t_{0}|\xi| \Phi(\xi)\right]=\exp \left[-c t_{0}|\xi|\right], \\
& |\xi-\hat{\xi}|+\left|v_{t_{0}}(\xi)\right| \geqq \varepsilon(|\xi|+|\hat{\xi}|), \quad \varepsilon>0,
\end{aligned}
$$

if $\xi \in \Gamma_{1}$ and $|\xi| \geqq 1$. It follows from (1.4.4)-(1.4.8) that

$$
\begin{aligned}
\left|\left\langle E(x), \exp [-i x \cdot \hat{\xi}] \chi_{N}(x)\right\rangle\right| & \\
= & \left|(2 \pi)^{-n} \int \hat{\chi}_{N}(\hat{\xi}-\zeta) P(\zeta)^{-1} d \zeta\right| \leqq C(C N)^{N}(1+|\hat{\xi}|)^{-N}, \\
& \hat{\xi} \in \Gamma_{2}, \quad \zeta=\xi-i v_{t_{0}}(\xi), \quad \xi \in \boldsymbol{R}^{n}, \quad N=1,2, \cdots .
\end{aligned}
$$

This proves the theorem.

Q.E.D.

Theorem 1.4.3. Let $\left(x^{0}, \xi^{0}\right) \in T^{*}\left(\boldsymbol{R}^{n}\right) \backslash 0$. Then we have $\left(x^{0}, \xi^{0}\right) \notin W F^{\left(1 / \delta\left(\xi \xi^{0}\right)\right)}$ $(E)$, where $\left(x^{0}, \xi^{0}\right) \notin W F^{(\infty)}(E)$ if $\delta\left(\xi^{0}\right)=0$.

Proof. Let $\Gamma$ and $\Gamma_{1}$ be conic neighborhoods of $\xi^{0}$ and $\Gamma_{1} \subset \Gamma$. Moreover 
let $\Phi(\xi)$ be as described in the proof of Theorem 1.4.2. Put

$$
v_{t}(\xi)=\gamma_{0}(1+|\xi|)^{\delta(P)} \vartheta+\gamma_{0} t \Phi(\xi)\left\{(1+|\xi|)^{\delta\left(\xi^{0}\right)}-(1+|\xi|)^{\delta(P)}\right\} \vartheta, \quad 0 \leqq t \leqq 1 .
$$

Then it follows from Lemma 1.2.6 and the definition of $\delta\left(\xi^{0}\right)$ that

$$
\left|P\left(\xi-i v_{t}(\xi)\right)\right|^{-1} \leqq C(1+|\xi|)^{a}, \quad \xi \in \boldsymbol{R}^{n}, \quad 0 \leqq t \leqq 1,
$$

for some constants $C$ and $a$. Let $\phi \in \mathscr{D}^{\left({ }^{\left(x_{0}\right)}\right.}$ and let $\Gamma_{2}\left(\Subset \Gamma_{1}\right)$ be a conic neighborhood of $\xi^{0}$. When $\hat{\xi} \in \Gamma_{2}$, we have

$$
|\xi-\hat{\xi}| \geqq \varepsilon(|\xi|+|\hat{\xi}|), \quad \varepsilon>0, \quad \text { if } \xi \notin \Gamma_{1} \quad \text { or } \quad|\xi| \geqq 2|\hat{\xi}|
$$

and $v_{1}(\xi)=\gamma_{0}(1+|\xi|)^{\delta\left(\xi^{0}\right)} \vartheta$ if $\xi \in \Gamma_{1}$ and $|\xi| \geqq 1$. Therefore Proposition 1.3.1 implies that

$$
|\langle E(x), \exp [-i x \cdot \hat{\xi}] \phi(x)\rangle| \leqq C \exp \left[C \mid \hat{\xi}^{\mid \delta\left(\xi^{0}\right)}\right] \quad \text { if } \hat{\xi} \in \Gamma_{2} \quad \text { and } \quad \delta\left(\xi^{0}\right)>0 .
$$

If $\delta\left(\xi^{0}\right)=0$, then we easily obtain

$$
|\langle E(x), \exp [-i x \cdot \hat{\xi}] \phi(x)\rangle| \leqq C(1+|\xi|)^{b}, \quad \hat{\xi} \in \Gamma_{2},
$$

for some constants $C$ and $b$. This completes the proof.

Q.E.D.

THEOREM 1.4.4. Let $1<\kappa_{1} \leqq \kappa_{0} \equiv 1 / \delta(P), \kappa_{1} \leqq \kappa \leqq \infty, f \in \mathscr{D}^{\left(\kappa_{1}\right)^{\prime}}\left(\right.$ resp. $\left.\mathscr{D}^{\left\{\kappa_{1}\right\}^{\prime}}\right)$ with $\operatorname{supp} f \subset\left\{x \in \boldsymbol{R}^{n} ; x \cdot \vartheta \geqq 0\right\}$ and $\xi \in \boldsymbol{R}^{n} \backslash\{0\}$. Then we have

$$
\begin{aligned}
& \left.W F_{(\kappa)}(E * f)\right|_{\xi}\left(\text { resp. }\left.W F_{\{k\}}(E * f)\right|_{\xi}\right) \\
& \subset\left\{\begin{array}{c}
\left\{x \in \boldsymbol{R}^{n} ; x-y \in \Gamma\left(P_{m \xi}, \vartheta\right)^{*},(y, \xi) \in W F_{(1 / \delta(\xi))}(f) \text { for some } y \in \boldsymbol{R}^{n}\right\} \\
\left\{x \in \boldsymbol{R}^{n} ; x-y \in \Gamma\left(P_{m \xi}, \vartheta\right)^{*},(y, \xi) \in W F_{(\kappa)}(f)\left(\text { resp. } W F_{\{k\}}(f)\right)\right. \\
\text { for some } \left.y \in \boldsymbol{R}^{n}\right\} \quad \text { if } \kappa<1 / \delta(\xi),
\end{array}\right. \\
& \left.W F^{(\kappa)}(E * f)\right|_{\xi}\left(\text { resp. }\left.W F^{\{\alpha\}}(E * f)\right|_{\xi}\right) \\
& \subset\left\{\begin{array}{r}
\left\{x \in \boldsymbol{R}^{n} ; x-y \in \Gamma\left(P_{m \xi}, \vartheta\right)^{*},(y, \xi) \in W F_{(1 / \delta(\xi))}(f) \text { for some } y \in \boldsymbol{R}^{n}\right\} \\
\text { if } \kappa>1 / \delta(\xi)(\text { resp. } \kappa \geqq 1 / \delta(\xi)), \\
\left\{x \in \boldsymbol{R}^{n} ; x-y \in \Gamma\left(P_{m \xi}, \vartheta\right)^{*},(y, \xi) \in W F^{(\kappa)}(f)\left(\operatorname{resp} . W F^{\{k\}}(f)\right)\right. \\
\text { for some } \left.y \in \boldsymbol{R}^{n}\right\} \quad \text { if } \kappa \leqq 1 / \delta(\xi)(\text { resp. } \kappa<1 / \delta(\xi)) .
\end{array}\right.
\end{aligned}
$$

Here $\left.W F^{(\infty)}(E * f)\right|_{\xi}=\left.W F^{\{\infty\}}(E * f)\right|_{\xi} \subset\left\{x \in \boldsymbol{R}^{n} ; x-y \in \Gamma\left(P_{m \xi}, \vartheta\right)^{*},(y, \xi) \in W F^{(\infty)}(f)\right.$ for some $\left.y \in \boldsymbol{R}^{n}\right\}$ if $\delta(\xi)=0$.

Proof. The theorem easily follows from Theorems 1.4.2 and 1.4.3 and Lemma 1.3.11. Then in order to estimate $\left.W F_{\{k\}}(E * f)\right|_{\xi}$ and $\left.W F^{\{k\}}(E * f)\right|_{\xi}$ for $\kappa \geqq 1 / \delta(\xi)$, we use the fact that 


$$
\begin{aligned}
& \left.\left.W F_{\{k\}}(E * f)\right|_{\xi} \subset W F_{(\kappa)}(E * f)\right|_{\xi}, \\
& \left.\left.W F^{\{\alpha\}}(E * f)\right|_{\xi} \subset W F^{(s)}(E * f)\right|_{\xi}, \quad s>\kappa .
\end{aligned}
$$

The finite set $\left\{\delta(\xi) ; \xi \in \boldsymbol{R}^{n} \backslash\{0\}\right\}$ can be written in the form

$$
\left\{1 / \delta(\xi) ; \xi \in \boldsymbol{R}^{n} \backslash\{0\}\right\}=\left\{s_{j} ; 1 \leqq j \leqq l\right\}, \quad 1<\kappa_{0}=s_{1}<s_{2}<\cdots<s_{l}=\infty .
$$

Define

$$
\begin{aligned}
K_{j}= & \left\{((x, \xi),(y, \xi)) ; x-y \in \Gamma\left(P_{m \xi}, \vartheta\right)^{*}, \xi \in \boldsymbol{R}^{n} \backslash\{0\} \text { and } 1 / \delta(\xi)=s_{j}\right\}, \\
& 1 \leqq j \leqq l, \\
K= & \bigcup_{j=1}^{l} K_{j}=\left\{((x, \xi),(y, \xi)) ; x-y \in \Gamma\left(P_{m \xi}, \vartheta\right)^{*} \text { and } \xi \in \boldsymbol{R}^{n} \backslash\{0\}\right\}
\end{aligned}
$$

Corollary 1.4.5. Denote by $\omega(\kappa)($ resp. $\omega\{\kappa\})$

$$
\max \left\{j ; \kappa>s_{j}\left(\text { resp. } \kappa \geqq s_{j}\right)\right\},
$$

where $s_{0}=1$. Then we have

$$
\begin{aligned}
& W F_{*_{k}}(E * f) \subset \bigcup_{k=1}^{\omega_{\kappa}^{*}} K_{k} \circ W F_{\left(s_{k}\right)}(f) \cup \bigcup_{k=\omega_{*}^{*}+1}^{l} K_{k} \circ W F_{*_{k}}(f), \\
& W F^{*_{\kappa}}(E * f) \subset \bigcup_{k=1}^{\omega_{*}} K_{k} \circ W F_{\left(s_{k}\right)}(f) \cup \bigcup_{k=\omega_{*}^{*} *_{k}}^{l} K_{k} \circ W F^{*_{k}}(f),
\end{aligned}
$$

where $\omega^{*} \kappa$ denotes either $\omega(\kappa)$ or $\omega\{\kappa\}$.

Corollary 1.4.6. We have the following: (i) If $\left.W F_{*_{k}}(f)\right|_{\xi}=\emptyset$ for $\kappa<$ $1 / \delta(\xi), \xi \in \boldsymbol{R}^{n} \backslash\{0\}$ and $\left.W F_{(1 / \delta(\xi))}(f)\right|_{\xi}=\emptyset$ for $\kappa \geqq 1 / \delta(\xi), \xi \in \boldsymbol{R}^{n} \backslash\{0\}$, then $W F_{*_{x}}(E * f)$ $=\emptyset$, that is, $E * f \in \mathscr{E}^{*_{\kappa}}$. (ii) If $\left.W F^{(\kappa)}(f)\right|_{\xi}=\emptyset$ (resp. $\left.\left.W F^{\{k\}}(f)\right|_{\xi}=\emptyset\right)$ for $\kappa \leqq 1 / \delta(\xi)$ (resp. $\kappa<1 / \delta(\xi)), \xi \in \boldsymbol{R}^{n} \backslash\{0\}$ and $\left.W F_{(1 / \delta(\xi))}(f)\right|_{\xi}=\emptyset$ for $\kappa>1 / \delta(\xi)($ resp. $\kappa \geqq 1 / \delta(\xi))$, $\xi \in \boldsymbol{R}^{n} \backslash\{0\}$, then $W F^{(x)}(E * f)=\emptyset$ (resp. WF $\left.F^{\{x\}}(E * f)=\emptyset\right)$, that is, $E * f \in \mathscr{D}^{(x)}$ (resp. $\left.\mathscr{D}^{\{\}^{\prime}}\right)$. Here $W F^{(\infty)}(E * f)=\emptyset$ if $\left.W F^{(\infty)}(f)\right|_{\xi}=\emptyset$ for $\delta(\xi)=0, \xi \in \boldsymbol{R}^{n} \backslash\{0\}$ and $\left.W F_{(1 / \delta(\xi))}(f)\right|_{\xi}=\emptyset$ for $\delta(\xi)>0, \xi \in \boldsymbol{R}^{n} \backslash\{0\}$.

\subsection{Some remarks}

In $\S 1.4$ we have given the outer estimates of the generalized wave front sets of the solutions. However, we could not give non-trivial inner estimates of the generalized wave front sets, which correspond to the localization theorem in [1]. The following lemma is not really satisfactory.

Lemma 1.5.1. Let $\left(x^{0}, \xi^{0}\right) \in T^{*}\left(\boldsymbol{R}^{n}\right) \backslash 0$ and $\eta(s)=s^{-1}\left(\xi^{0}+\sum_{j=1}^{\infty} s^{j / l} \xi^{j}\right), \xi^{j} \in \boldsymbol{C}^{n}$, $j=1,2, \cdots, l \in N$. Define a polynomial $P(\zeta ; \eta(s)) \neq 0$ in $\zeta$ by

$$
P(\eta(s)+\zeta)=s^{-r(\eta)}\{P(\zeta ; \eta(s))+o(1)\}, \quad s \rightarrow+0 .
$$

Assume that the following conditions hold: (i) The principal part of $P(\zeta ; \eta(s))$ 
is hyperbolic with respect to $\vartheta$. (ii) $x^{0} \in \operatorname{supp} E(P(\cdot ; \eta(s)), \vartheta ; x)$. (iii) $x^{0} \cdot \operatorname{Im} \eta(s)$ $\leqq-s^{-\nu}(c+o(1)), s \rightarrow+0, \nu \geqq 0$, where $c$ is a positive number if $\nu>0$ and $c$ is any constant if $\nu=0$. Then we have $\left(x^{0}, \xi^{0}\right) \in W F^{\{1 / \nu\}}(E(P, \vartheta ; x))$ if $\nu>0$ and $\left(x^{0}, \xi^{0}\right)$ $\in W F(E)=W F_{(\infty)}(E)$ if $\nu=0$.

Proof. Put $F(x, s)=\exp [-i x \cdot \eta(s)] E(P, \vartheta ; x)$. Then we have

$$
\begin{aligned}
\mathscr{F}[\phi E](\eta(s))=\mathscr{F}[\phi F](0)=s^{r(\eta)}\{\langle E(P(\cdot ; \eta(s)), \vartheta ; x), \phi(x)\rangle+o(1)\}, \\
s \rightarrow+0, \quad \phi \in \mathscr{D}^{\left(\kappa_{1}\right)}, \quad 1<\kappa_{1} \leqq 1 / \delta(P) .
\end{aligned}
$$

Now we assume that $\nu>0$ and $\left(x^{0}, \xi^{0}\right) \notin W F^{\{1 / \nu\}}(E)$. Then for sufficiently small neighborhood $U$ of $x^{0}$ we have

$$
|\mathscr{F}[\phi E](\eta(s))| \leqq C \exp \left[-c s^{-\nu} / 4\right], \quad 0<s \leqq s_{0}, \quad \phi \in \mathscr{D}^{\left(\kappa_{1}\right)}(U),
$$

which is a contradiction to (1.5.1). When $\nu=0$, we can similarly prove the lemma.

Q.E.D.

ExAMPle 1.5.2. Let $P(\xi)=\xi_{1}\left(\xi_{1}-\xi_{2}\right)+\xi_{3}$ and $\xi^{0}=(0,0,1)$. Then we have

$$
\begin{aligned}
\left.W F^{\{2\}}(E(P, \vartheta ; x))\right|_{\xi^{0}}=\left.W F_{A}(E)\right|_{\xi^{0}}=\left\{x \in R^{3} ;\right. & x=\alpha(1,0,0) \\
& +\beta(1,-1,0) \text { and } \alpha, \beta \geqq 0\}
\end{aligned}
$$

and $W F^{(2)}(E(P, \vartheta ; x))=\emptyset$. In fact we take $\eta(s)=s^{-1} \xi^{0}-i s^{-1 / 2}(a, b, 0)$, where $a>0, b \in R$ and $1-a(a-b)=0$. Then $\eta(s)$ satisfies the conditions of Lemma 1.5.1 with $x^{0}=\alpha(1,0,0)+\beta(1,-1,0)$ for $\alpha, \beta>0$ and $\nu=1 / 2$. Since $W F^{\{x\}}(f)$ is closed in $T^{*}\left(\boldsymbol{R}^{n}\right) \backslash 0$, we have (1.5.2).

ExAmple 1.5.3. Let $P(\xi)=\xi_{1}^{3}+\xi_{2} \xi_{3}$ and $\xi^{0}=(0,0,1)$. Put $\xi^{j}=(0,1 / j, 1)$, $j=1,2, \cdots$, and $\eta^{j}(s)=s^{-1} \xi^{j}+s^{-2 / 3} \omega j^{-1 / 3} \vartheta$, where $\omega=(1-i \sqrt{3}) / 2, \omega^{3}=1$. If $\xi^{0}$ and $\eta(s)$ are replaced by $\xi^{j}$ and $\eta^{j}(s)$, respectively, the conditions of Lemma 1.5.1 are satisfied when $x^{0}=\alpha \vartheta$ for $\alpha \geqq 0$ and $\nu=2 / 3$. Thus we have

$$
\left.W F^{\{3 / 2\}}(E)\right|_{\xi j}=\left.W F_{A}(E)\right|_{\xi^{j}}=\left\{x \in \boldsymbol{R}^{3} ; x=\alpha \vartheta, \alpha \geqq 0\right\} .
$$

The closedness of the wave front set implies that

$$
\left.W F^{\{3 / 2\}}(E)\right|_{\xi^{0}}=\left.W F_{A}(E)\right|_{\xi^{0}}=\left\{x \in R^{3} ; x=\alpha \vartheta, \alpha \geqq 0\right\} .
$$

Lemma 1.5.4. Let $\xi^{0} \in \boldsymbol{R}^{n} \backslash\{0\}$. If $\max \left(\delta\left(\xi^{0}\right), \delta\left(-\xi^{0}\right)\right)=0$, then $P_{\hat{\xi}^{0}}(\xi)$ is hyperbolic with respect to $\vartheta$ and the principal part of $P_{\xi^{0}}(\xi)$ is $P_{m \xi}(\xi)$. Therefore we have

$$
\begin{aligned}
& \left.\left.\operatorname{supp} E\left(P_{\xi_{0} 0}, \vartheta ; x\right) \subset W F(E(P, \vartheta ; x))\right|_{\xi_{0}} \subset W F_{A}(E(P, \vartheta ; x))\right|_{\xi^{0}} \\
& \subset \Gamma\left(P_{m \xi}, \vartheta\right)^{*}=\operatorname{ch}\left[\operatorname{supp} E\left(P_{\xi 0}, \vartheta ; x\right)\right] .
\end{aligned}
$$

Proof. Write 


$$
\begin{aligned}
P_{m}\left(s^{-1} \xi^{0}+\zeta\right) & =s^{-p}\left(P_{m \xi^{0}}(\zeta)+o(1)\right), & & s \rightarrow+0, \\
Q\left(s^{-1} \xi^{0}+\zeta\right) & =s^{-q}\left(Q_{\xi^{0}}(\zeta)+o(1)\right), & & s \rightarrow+0 .
\end{aligned}
$$

Then we have $p \geqq q$, since $n_{+}\left(P_{m}, Q_{j} ; \xi^{0}\right)=0$. If $p>q$, then $P_{\xi^{0}}(\zeta)=P_{m \xi^{0}}(\zeta)$. If $p=q$, then $\operatorname{deg} P_{m \xi^{0}}=m-p>\operatorname{deg} Q_{\xi^{0}}$. Thus the principal part of $P_{\xi 0}(\zeta)$ is $P_{m \xi 0}(\zeta)$. By the assumption there are a conic neighborhood $\Gamma$ of $\xi^{0}$ and a positive number $\gamma_{0}$ such that $P(\xi-i \gamma \vartheta) \neq 0, \xi \in \Gamma, \gamma>\gamma_{0}$. If we assume that $P_{\xi 0}\left(\xi^{1}-i \gamma_{1} \vartheta\right)=0$ for some $\gamma_{1}>\gamma_{0}$ and $\xi^{1} \in R$, Rouché's theorem leads us to a contradiction. This proves the lemma, since (1.5.3) is an immediate consequence of Lemma 1.5.1.

Q.E.D.

\section{Chapter II}

\section{Operators with variable lower-order coefficients}

\subsection{Construction of solutions}

Let $\xi^{0} \in \boldsymbol{R}^{n} \backslash\{0\}$. Define

$$
\begin{aligned}
n_{j}\left(\xi^{0}\right) & =\max _{x \in \boldsymbol{R}^{n}} n_{+}\left(P_{m}, Q_{j}(x, \cdot) ; \xi^{0}\right), \\
\kappa\left(\xi^{0}\right) & =\min _{0 \leqq j \leqq m-1}\left(m-j+n_{j}\left(\xi^{0}\right)\right) / n_{j}\left(\xi^{0}\right), \\
\kappa_{0} & =\min _{|\xi|=1} \kappa(\xi),
\end{aligned}
$$

where $\kappa\left(\xi^{0}\right)=\infty$ if $n_{j}\left(\xi^{0}\right)=0$. Here we have used the fact that the set

$$
\left\{n\left(P_{m}, Q_{j}(x, \cdot) ; \xi\right) ;|\xi|=1, x \in R^{n} \text { and } 0 \leqq j \leqq m-1\right\}
$$

is finite. Indeed, applying the same argument as in the proof of Lemma 1.1.3 to the system

$$
\left\{\begin{array}{l}
\phi\left|P_{m}(\xi-i \vartheta)\right|^{2}-\left|\sum_{|\alpha| \leqq m-1} a_{\alpha}(\xi-i \vartheta)^{\alpha}\right|=0, \quad a_{\alpha} \in C, \\
|\xi|^{2}=r^{2}, \quad r>0, \\
\left|\xi-r \xi^{0}\right| \leqq \varepsilon^{2} r^{2}, \quad 0<\varepsilon \leqq \varepsilon_{0}, \quad\left|\xi^{0}\right|=1, \quad \xi^{0} \in \boldsymbol{R}^{n},
\end{array}\right.
$$

we can prove this fact. Put

$$
\begin{aligned}
V(P)= & V_{0}(P) \oplus V_{1}(P) \oplus \cdots \oplus V_{m-1}(P), \\
V_{j}(P)= & \{p(\xi) \in C[\xi] ; p(\xi) \text { is homogeneous of degree } j \\
& \text { and } \left.n_{+}\left(P_{m}, p ; \eta\right) \leqq n_{j}(\eta) \text { for all } \eta \in \boldsymbol{R}^{n} \backslash\{0\}\right\} .
\end{aligned}
$$

Let $\left\{p^{j}\right\}$ be a basis of the finite dimensional vector sapce $V(P)$. Here we can choose $p^{j}$ to be homogeneous. Let $\kappa_{1}>1$ and assume that the coefficients of $Q(x, D)$ belong to $\mathscr{E}^{*_{x_{1}}}$. Then the following lemma is obvious. 
LEMMA 2.1.1. We can write

$$
Q(x, \xi)=\sum_{j} q_{j}(x) p^{j}(\xi), \quad q_{j}(x) \in \mathscr{E}^{*_{\kappa_{1}}} .
$$

Moreover the $q_{j}(x)$ are in $\mathscr{D}^{*_{x_{1}}}$ if the coefficients of $Q(x, D)$ are in $\mathscr{D}^{*_{\kappa_{1}}}$.

We assume that

$$
1<\kappa_{1}<\kappa_{0}
$$

Let $\kappa_{1} \leqq \kappa \leqq \kappa_{0}$ when $*_{\kappa_{1}}=\left(\kappa_{1}\right)$ and $\kappa_{1} \leqq \kappa<\kappa_{0.3}^{\pi}$ when $*_{\kappa_{1}}=\left\{\kappa_{1}\right\}$. Consider the Cauchy problem

$$
\left\{\begin{array}{l}
P(x, D) u(x)=f(x), \\
\operatorname{supp} u(x) \subset\left\{x \in R^{n} ; x_{1} \geqq t\right\},
\end{array}\right.
$$

where $f \in \mathscr{D}^{*^{\prime}}$, supp $f \subset\left\{x \in \boldsymbol{R}^{n} ; x_{1} \geqq t\right\}$ and $t$ is a fixed real number. First we construct a solution of (2.1.4) by successive iteration when the $q_{j}(x)$ are in $\mathscr{D}^{*_{\kappa_{1}}}$ and $f \in \mathscr{E}^{*_{r^{\prime}}}$. Define $u_{l}, l=0,1,2, \cdots$, by

$$
\begin{aligned}
& P_{m}(D) u_{0}(x)=f(x), \\
& P_{m}(D) u_{l+1}(x)=-Q(x, D) u_{l}(x), \\
& \text { supp } u_{l} \subset \operatorname{supp} f+\Gamma\left(P_{m}, \vartheta\right)^{*} .
\end{aligned}
$$

It is obvious that $u_{l} \in \mathscr{D}^{*_{r^{\prime}}}$.

Lemma 2.1.2. Assume that the condition (A) is satisfied, the $q_{j}(x)$ are in $\mathscr{D}^{\left(\kappa_{1}\right)}\left(\right.$ resp. $\left.\mathscr{D}^{\left\{\kappa_{1}\right\}}\right)$ and that $f \in \mathscr{E}^{(x)^{\prime}}\left(\right.$ resp. $\left.\mathscr{E}^{\{r\}^{\prime}}\right)$ with $\operatorname{supp} f \subset\left\{x \in \boldsymbol{R}^{n} ; x_{1} \geqq 0\right\}$. Then we have

$$
\begin{aligned}
& \left|\hat{u}_{l}\left(\xi-i \gamma(1+|\xi|)^{1 / \kappa_{0}} \vartheta\right)\right| \\
& \quad \leqq C_{0}\left(C \gamma^{-\mu}\right)^{l+1} \exp \left[L\left|\xi-i \gamma(1+|\xi|)^{1 / \kappa_{0}} \vartheta\right|^{1 / \kappa}\right], \quad \gamma \geqq 1, \quad \xi \in \boldsymbol{R}^{n},
\end{aligned}
$$

for some constants $L$ and $C_{0}$ (resp. for every $L>0$ and some constant $C_{0}$ depending on $L$ ), where $C$ is a positive constant and

$$
\begin{aligned}
& \mu=\min _{0 \leqq j \leqq m-1}\left(m-j-n_{j}(P)\left(\kappa_{1}-1\right)\right)>0, \\
& n_{j}(P)=\max _{|\xi|=1} n_{j}(\xi) .
\end{aligned}
$$

Thus we can define $u(x) \in \mathscr{D}^{(x)^{\prime}}\left(\right.$ resp. $\left.\mathscr{D}^{\{r\}^{\prime}}\right)$ by

$$
\hat{u}\left(\xi-i \gamma(1+|\xi|)^{1 / \kappa_{0}} \vartheta\right)=\sum_{l=0}^{\infty} \hat{u}_{l}\left(\xi-i \gamma(1+|\xi|)^{1 / \kappa_{0}} \vartheta\right), \quad \gamma^{\mu}>C,
$$

that is,

$$
\begin{gathered}
\langle u(x), \phi(x)\rangle=(2 \pi)^{-n} \int \hat{u}(\zeta) \hat{\phi}(-\zeta) d \zeta, \quad \zeta=\xi-i \gamma(1+|\xi|)^{1 / \kappa_{0}} \vartheta \\
\xi \in R^{n}, \quad \phi \in \mathscr{D}^{(x)} \quad\left(\text { resp. } \mathscr{D}^{\{n\}}\right) .
\end{gathered}
$$


Then $u(x)$ is a solution of (2.1.4) and

$$
\operatorname{supp} u(x) \subset \operatorname{supp} f(x)+\Gamma\left(P_{m}, \vartheta\right)^{*} .
$$

REMARK. When $\kappa_{0}=\infty$ we proved the lemma for $\kappa_{1} \leqq \infty$ (see [20]).

Proof. From the above remark it suffices to prove the lemma in the case $\kappa_{0}<\infty$. We have

$$
\begin{aligned}
\hat{u}_{l}(\xi- & \left.i \gamma(1+|\xi|)^{1 / \kappa_{0}} \vartheta\right) \\
= & (2 \pi)^{-l n} \sum_{j_{1}, \cdots, j_{l}}(-1)^{l} P_{m}\left(\xi-i \gamma(1+|\xi|)^{1 / \kappa_{0}} \vartheta\right)^{-1} \\
& \times \int d \zeta^{1} \hat{q}_{j_{1}}\left(\zeta^{1}\right) p^{j_{1}}\left(\xi-i \gamma(1+|\xi|)^{1 / \kappa_{0}} \vartheta-\zeta^{1}\right) P_{m}\left(\xi-i \gamma(1+|\xi|)^{1 / \kappa_{0}} \vartheta-\zeta^{1}\right)^{-1} \\
& \times\left\{\int d \zeta^{2} \cdots\right. \\
& \times\left\{\int d \zeta^{l} \hat{q}_{j_{l}}\left(\zeta^{l}\right) p^{j_{l}}\left(\xi-i \gamma(1+|\xi|)^{1 / \kappa_{0}} \vartheta-\zeta^{1}-\cdots-\zeta^{l}\right)\right. \\
& \times P_{m}\left(\xi-i \gamma(1+|\xi|)^{1 / \kappa_{0}} \vartheta-\zeta^{1}-\cdots-\zeta^{l}\right)^{-1} \\
& \left.\left.\times \hat{f}\left(\xi-i \gamma(1+|\xi|)^{1 / \kappa_{0}} \vartheta-\zeta^{1}-\cdots-\zeta^{l}\right)\right\} \cdots\right\},
\end{aligned}
$$

where $\zeta^{j}=\eta^{j}+i a\left(1+\left|\eta^{j}\right|\right)^{1 / \kappa i} \vartheta, \eta^{j} \in R^{n}, 1 \leqq j \leqq l$, and $a>0$ is to be determined later. Put

$$
J_{k}^{j}=\left|p^{j}\left(\xi-i \gamma(1+|\xi|)^{1 / \kappa_{0}} \vartheta-\zeta^{1}-\cdots-\zeta^{k}\right) P_{m}\left(\xi-i \gamma(1+|\xi|)^{1 / \kappa_{0}} \vartheta-\zeta^{1}-\cdots-\zeta^{k}\right)^{-1}\right| .
$$

Then we have

$$
J_{k}^{j} \leqq C \gamma^{-\mu}, \quad \gamma \geqq 1
$$

In fact,

$$
\begin{aligned}
J_{k}^{j} \leqq & C\left\{\gamma(1+|\xi|)^{1 / \kappa_{0}}+a \sum_{h=1}^{k}\left(1+\left|\eta^{h}\right|\right)^{1 / \kappa_{1}}\right\}^{\nu-m} \\
& \times\left[1+\left\{\gamma(1+|\xi|)^{1 / \kappa_{0}}+a \sum_{h=1}^{k}\left(1+\left|\eta^{h}\right|\right)^{1 / \kappa_{1}}\right\}^{-1}\left|\xi-\eta^{1}-\cdots-\eta^{k}\right|\right]^{n_{\mathcal{\nu}}(P)}, \quad \gamma>0,
\end{aligned}
$$

where $\operatorname{deg} p^{j}=\nu$. Thus

$$
J_{k}^{j} \leqq C \gamma^{\nu-m}, \quad \gamma \geqq 1, \quad \text { if }\left|\eta^{1}\right|+\cdots+\left|\eta^{k}\right| \leqq|\xi|,
$$

since $n_{\nu}(P)-\left(m-\nu+n_{\nu}(P)\right) / \kappa_{0} \leqq 0$. Moreover we have

$$
\begin{aligned}
J_{k}^{j} & \leqq C\left[\gamma^{\nu-m}+\gamma^{-\mu}\left\{a \sum_{h=1}^{k}\left(1+\left|\eta^{h}\right|\right)^{1 / \kappa_{1}}\right\}^{\nu-m+\mu+\left(\kappa_{1}-1\right) n_{\nu}(P)}\right] \\
& \leqq C \gamma^{-\mu}, \quad \gamma \geqq 1, \quad \text { if }\left|\eta^{1}\right|+\cdots+\left|\eta^{k}\right| \geqq|\xi|,
\end{aligned}
$$

since $\left(\left|\eta^{1}\right|+\cdots+\left|\eta^{k}\right|\right)^{1 / \kappa_{1}} \leqq\left|\eta^{1}\right|^{1 / \kappa_{1}}+\cdots+\left|\eta^{k}\right|^{1 / \kappa_{1}}, \nu-m+\left(\kappa_{1}-1\right) n_{\nu}(P)+\mu \leqq 0$ and $m$ $-\nu \geqq \mu$. (2.1.9) and (2.1.10) give (2.1.8). Note that $\mu=n(P)\left(\kappa_{0}-\kappa_{1}\right)$ if $n(P) \neq 0$, 
$\mu=1$ if $n(P)=0$, where $n(P)=\max _{0 \leqq j \leqq m-1} n_{j}(P)$. Thus $\mu>0$. Now Proposition 1.3.1 implies that

$$
\left|\hat{q}_{j}(\zeta)\right| \leqq C_{1} \exp \left[A|\operatorname{Im} \zeta|-B_{1}|\operatorname{Re} \zeta|^{1 / \kappa_{1}}\right]
$$

for every $B_{1}>0$ and some $C_{1}$ depending on $B_{1}$ (resp. for some $B_{1}>0$ and $C_{1}$ ), and

$$
|\hat{f}(\xi-i \gamma \vartheta)| \leqq C_{2} \exp \left[B_{2}|\xi-i \gamma \vartheta|^{1 / \kappa}\right], \quad \gamma>0,
$$

for some $B_{2}$ and $C_{2}$ (resp. for every $B_{2}>0$ and some $C_{2}$ depending on $B_{2}$ ). Choose $a>0$ and $B_{1}>0$ (resp. $\left.B_{2}>0\right)$ so that $B_{1} \geqq 2\left(a A+\left(1+a^{1 / r}\right) B_{2}\right)$. Then it follows from (2.1.7) and (2.1.8) that

$$
\begin{aligned}
& \left|\hat{u}_{l}\left(\xi-i \gamma(1+|\xi|)^{1 / \kappa_{0}} \vartheta\right)\right| \leqq C\left|P_{m}\left(\xi-i \gamma(1+|\xi|)^{1 / \kappa 0} \vartheta\right)\right|^{-1} \\
& \times\left(C \gamma^{-\mu}\right)^{l} \int d \eta^{1} \cdots d \eta^{l} C_{1}^{l} C_{2} \exp \left[a A \sum_{k=1}^{l}\left(1+\left|\eta^{k}\right|\right)^{1 / \kappa_{1}}\right. \\
& -B_{1} \sum_{k=1}^{l}\left|\eta^{k}\right|^{1 / \kappa_{1}}+B_{2}\left|\xi-i \gamma(1+|\xi|)^{1 / \kappa_{0}} \vartheta-\eta^{1}-\cdots-\eta^{l}\right|^{1 / k} \\
& \left.+a^{1 / \kappa} B_{2} \sum_{k=1}^{l}\left(1+\left|\eta^{k}\right|\right)^{1 /\left(\kappa \kappa_{1}\right)}\right] \\
& \leqq C C_{2}\left|P_{m}\left(\xi-i \gamma(1+|\xi|)^{1 / \alpha_{0}} \vartheta\right)\right|^{-1} \\
& \times\left(C C_{1} \gamma^{-\mu}\right)^{l} \exp \left[B_{2}\left|\xi-i \gamma(1+|\xi|)^{1 / \kappa_{0}} \vartheta\right|^{1 / \kappa}\right], \quad \gamma \geqq 1 .
\end{aligned}
$$

Here we have used the fact that $(a+b)^{1 / \lambda} \leqq a^{1 / \lambda}+b^{1 / \lambda}$ if $a, b \geqq 0$ and $\lambda \geqq 1$. This proves the first part of the lemma. It is obvious that $\hat{u}\left(\xi-i \gamma(1+|\xi|)^{1 / \alpha_{0}} \vartheta\right)$ is well-defined for $\gamma>\gamma_{0}=\left(C C_{1}\right)^{1 / \mu}$ and that

$$
\left|\hat{u}\left(\xi-i \gamma(1+|\xi|)^{1 / \kappa_{0}} \vartheta\right)\right| \leqq C_{\gamma} \exp \left[L\left|\xi-i \gamma(1+|\xi|)^{1 / \kappa_{0}} \vartheta\right|^{1 / \kappa}\right], \quad \gamma>\gamma_{0},
$$

for some $L$ and $C_{\gamma}$ (resp. for every $L>0$ and some $C_{\gamma}$ depending on $L$ and $\gamma$ ). If $\phi \in \mathscr{D}^{(k)}\left(\right.$ resp. $\left.\mathscr{D}^{\{x\}}\right)$, then

$$
\left|\hat{\phi}\left(-\xi+i \gamma(1+|\xi|)^{1 / \kappa_{0}} \vartheta\right)\right| \leqq C \exp \left[A \gamma(1+|\xi|)^{1 / \kappa_{0}}-B|\xi|^{1 / \kappa}\right]
$$

for every $B>0$ and some $C$ (resp. for some $B>0$ and $C$ ). This implies that $u \in \mathscr{D}^{(k)^{\prime}}$ for $\kappa_{1} \leqq \kappa \leqq \kappa_{0}$ (resp. $u \in \mathscr{D}^{\{k\}^{\prime}}$ for $\kappa_{1} \leqq \kappa<\kappa_{0}$ ). The last assertion of the lemma is obvious.

Q.E.D.

Lemma 2.1.3. Let $P(x, D)$ be as described in Lemma 2.1.2. Assume that $f \in \mathscr{D}^{(x)}\left(\right.$ resp. $\left.\mathscr{D}^{\{x\}}\right)$ with $\operatorname{supp} f \subset\left\{x \in \boldsymbol{R}^{n} ; x_{1} \geqq 0\right\}$. Then the solution $u(x)$ of (2.1.4) defined by (2.1.6) belongs to $\mathscr{E}^{(x)}$ (resp. $\left.\mathscr{E}^{[x\}}\right)$.

REmark. The more general result will be given in Theorem 2.4.4.

Proof. Instead of (2.1.11) we have

$$
|\hat{f}(\xi-i \gamma \vartheta)| \leqq C_{2} \exp \left[-B_{2}|\xi|^{1 / r}\right], \quad \gamma>0,
$$


for every $B_{2}>0$ and some $C_{2}$ depending on $B_{2}$ (resp. for some $B_{2}>0$ and $C_{2}$ ). Choose $a>0$ and $B_{1}>0$ (resp. $B_{2}>0$ ) in the proof of Lemma 2.1.2 so that $B_{1} \geqq$ $2\left(a A+B_{2}\right)$. Here we have used the fact that (2.1.12) with $B_{2}^{\prime}$ holds if $B_{2}^{\prime}<B_{2}$. Repeating the same argument as in the proof of Lemma 2.1.2, we have

$$
\begin{aligned}
\left|\hat{u}_{l}\left(\xi-i \gamma(1+|\xi|)^{1 / \kappa_{0}} \vartheta\right)\right| \leqq & C C_{2}\left|P_{m}\left(\xi-i \gamma(1+|\xi|)^{1 / \kappa_{0}} \vartheta\right)\right|^{-1} \\
& \times\left(C_{1} C \gamma^{-\mu}\right)^{l} \exp \left[-B_{2}|\xi|^{1 / \kappa}\right], \quad \gamma \geqq 1 .
\end{aligned}
$$

Thus

$$
\left|\hat{u}\left(\xi-i \gamma(1+|\xi|)^{1 / \kappa_{0}} \vartheta\right)\right| \leqq C_{\gamma} \exp \left[-B_{2}|\xi|^{1 / \kappa}\right], \quad \gamma>\gamma_{0}=\left(C_{1} C\right)^{1 / \mu} .
$$

If $\phi \in \mathscr{D}^{(x)}\left(\right.$ resp. $\left.\mathscr{D}^{\{k\}}\right)$ and supp $\phi \subset\left\{x_{1} \geqq-\varepsilon\right\}, \varepsilon>0$, then

$$
|\hat{\phi}(\xi-\zeta)| \leqq C \exp \left[\varepsilon \gamma(1+|\eta|)^{1 / \kappa_{0}}-B|\xi-\eta|^{1 / \kappa}\right]
$$

for every $B>0$ and $C$ depending on $B$ (resp. for some $B>0$ and $C$ ), where $\zeta=$ $\eta-i \gamma(1+|\eta|)^{1 / \kappa_{0}} \vartheta$ and $\xi, \eta \in \boldsymbol{R}^{n}$. Therefore we have

$$
\begin{gathered}
|\mathscr{F}[\phi u](\xi)|=\left|(2 \pi)^{-n} \int \hat{\phi}(\xi-\zeta) \hat{u}(\zeta) d \zeta\right| \leqq C_{r} \exp \left[-B_{3}|\xi|^{1 / k}\right], \\
\zeta=\eta-i \gamma(1+|\eta|)^{1 / \kappa_{0}} \vartheta, \quad \eta \in \boldsymbol{R}^{n}, \quad \xi \in \boldsymbol{R}^{n},
\end{gathered}
$$

where $\varepsilon=1 / \gamma, \gamma>\gamma_{0}, B=B_{2}$ and $B_{3}=B_{2}-1$ (resp. where $B_{3}=\min \left(B / 2, B_{2} / 2\right)$ ). Here we have used the fact that

$$
\exp \left[\varepsilon \gamma(1+|\eta|)^{1 / \kappa_{0}}-B_{2}|\eta|^{1 / \kappa} / 2\right] \leqq C\left(\kappa, \kappa_{0}, \varepsilon \gamma, B_{2}\right) \quad \text { if } \kappa_{0}>\kappa . \quad \text { Q.E.D. }
$$

LEMMA 2.1.4. Put

$$
\begin{aligned}
\kappa\left(P, x ; \xi^{0}\right)= & \min _{0 \leqq j \leqq m-1}\left(m-j+n_{+}\left(P_{m}, Q_{j}(x, \cdot) ; \xi^{0}\right)\right) \\
& \times 1 / n_{+}\left(P_{m}, Q_{j}(x, \cdot) ; \xi^{0}\right), \quad x \in \boldsymbol{R}^{n}, \quad \xi^{0} \in \boldsymbol{R}^{n} \backslash\{0\} .
\end{aligned}
$$

Then we have

$$
\kappa\left(P, x ; \xi^{0}\right)=\kappa\left({ }^{t} P, x ; \xi^{0}\right),
$$

where ${ }^{t} P$ denotes the transposed operator of $P(x, D)$.

Proof. Put $\nu_{j}=\operatorname{deg} p^{j}$. Then

$$
{ }^{t} P(x, D) u(x)=(-1)^{m} P_{m}(D) u(x)+(-1)^{\nu_{j}} \sum_{j, \alpha} 1 / \alpha ! D^{\alpha} q_{j}(x) p^{j(\alpha)}(D) u(x) .
$$

From the definition of $n\left(P_{m}, p^{j} ; \xi^{0}\right)$, hyperbolicity of $P_{m}(\xi)$ and Lemma 3.1.5 in [7] it follows that there exists a conic neighborhood $\Gamma$ of $\xi^{0}$ such that

$$
\begin{aligned}
\left|p^{j(\alpha)}(\xi-i \vartheta)\right| & \leqq C \sup _{|\zeta| \leqq 1}\left|p^{j}(\xi+\zeta-i \vartheta)\right| \\
& \leqq C(1+|\xi|)^{n\left(P_{m}, p^{j} ; \xi 0\right)}\left|P_{m}(\xi-i \vartheta)\right|, \quad \xi \in \Gamma .
\end{aligned}
$$


This implies that $n\left(P_{m}, p^{j(\alpha)} ; \xi^{0}\right) \leqq n\left(P_{m}, p^{j} ; \xi^{0}\right)$. Thus we have

$$
\begin{gathered}
\left(m-\nu_{j}+|\alpha|+n_{+}\left(P_{m}, p^{j(\alpha)} ; \xi^{0}\right)\right) / n_{+}\left(P_{m}, p^{j(\alpha)} ; \xi^{0}\right) \\
\geqq\left(m-\nu_{j}+n_{+}\left(P_{m}, p^{j} ; \xi^{0}\right)\right) / n_{+}\left(P_{m}, p^{j} ; \xi^{0}\right),
\end{gathered}
$$

which proves $\kappa\left({ }^{t} P, x ; \xi^{0}\right) \geqq \kappa\left(P, x ; \xi^{0}\right)$. Since ${ }^{t}\left({ }^{t} P\right)=P$, the proof is complete.

Q.E.D.

By Lemmas 2.1.2-2.1.4 we can apply the same arguments as in Theorem 2.5 in [20] to obtain easily the following theorem.

THeOREm 2.1.5 (finite propagation property). Assume that the condition (A) is satisfied. If $u \in \mathscr{D}^{*_{k^{\prime}}}$ with supp $u \subset\left\{x_{1} \geqq t\right\}$ satisfies the equation $P(x, D)$ $u(x)=0$ in a neighborhood of $x^{0}-\Gamma\left(P_{m}, \vartheta\right)^{*}$, then $u=0$ in a neighborhood of $x^{0}$.

THEOREM 2.1.6. Under the condition (A) the Cauchy problem (2.1.4) has a unique solution u in $\mathscr{D}^{*^{\prime}}$.

\subsection{Necessary conditions for well-posedness}

In this section we shall prove the following theorem by the same arguments as in [11].

TheORem 2.2.1. Assume that the coefficients of $Q(x, D)$ are real analytic. If the Cauchy problem (2.1.4) has a unique solution $u(x)$ in $\mathscr{E}^{*_{x}}$ for every $f \in \mathscr{E} *_{x}$ with $\operatorname{supp} f \subset\left\{x_{1} \geqq t\right\}$ and every $t \in R$, then we have $\kappa \leqq \kappa_{0}$, where $\kappa_{0}$ is defined by (2.1.3).

REMARK. (i) Theorem 2.2.1 and the results in [11] imply that it is necessary and sufficient for the Cauchy problem (2.1.4) to be $\mathscr{E}^{(n)}$-well posed, $t \in \boldsymbol{R}$, that $\kappa \leqq \kappa_{0}$, if the coefficients of $Q(x, D)$ are real analytic. (ii) When $n \leqq 3$, we can prove that the condition that $\kappa<\kappa_{0}$ is necessary for the Cauchy problem to be $\mathscr{E}^{\{x\}}$-well posed (see the remark of Theorem A.1.8).

In this section we assume that the coefficients of $Q(x, D)$ are real analytic. Following Ivrii [11], we define the following

Definition 2.2.2. The Cauchy problem

$$
\left\{\begin{array}{l}
P(x, D) u(x)=f(x), \\
\operatorname{supp} u \subset \bar{\Omega}_{T}^{+}
\end{array}\right.
$$

is said to be weakly $\mathscr{E}^{[x\}}$-correct if for every $f \in \mathscr{D}^{\{x\}}\left(\Omega_{T}^{+}\right)$there is a solution $u$ in $\mathscr{D}^{\{r\}^{\prime}}(\Omega)$ satisfying (2.2.1) in $\Omega$, where $\Omega$ is an open domain in $\boldsymbol{R}^{n}, \Omega_{T}^{+}=$ $\left\{x \in \Omega ; x_{1} \geqq T\right\}, T \in R$ and $1<\kappa<\infty$. 
Introduce the semi norm

$$
|u ; K, \kappa, \theta|=\sum_{\alpha} \theta^{|\alpha|}\left|D^{\alpha} u ; L^{2}(K)\right| /\left(\alpha !(|\alpha| !)^{\kappa-1}\right),
$$

where $\theta>0,1<\kappa<\infty$ and $K$ is a compact set in $\boldsymbol{R}^{n}$. Define

$$
\mathscr{D}^{\{\kappa\}}(K, \theta)=\left\{u \in \mathscr{D}^{\{\kappa\}} ; \operatorname{supp} u \subset K,|u ; K, \kappa, \theta|<\infty\right\} .
$$

Then $\mathscr{D}^{[x\}}(K, \theta)$ is a Banach space and we have

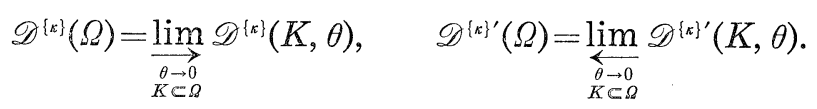

Lemma 2.2.3 ([11]). Assume that the Cauchy problem (2.2.1) is weakly

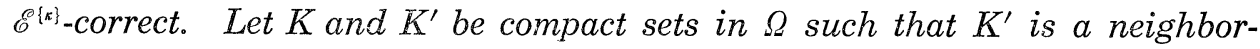
hood of $K$. Then there is a constant $t_{0}>T$ such that the following inequality holds for all $t \in\left(T, t_{0}\right], \theta>0, \tau>0$, with some constant $C=C_{\tau, \theta}$ :

$$
\left|u ; C\left(K_{t}^{-}\right)\right|^{2} \leqq C\left|u ; K_{t+\tau}^{\prime-}, \kappa, \theta\right|\left|P u ; K_{t+\tau}^{\prime-}, \kappa, \theta\right|
$$

for all $u \in \mathscr{D}^{[x\}}\left(\Omega_{T}^{+}\right)$, where $K_{t}^{-}=\left\{x \in K ; x_{1} \leqq t\right\}$.

Let $\hat{\eta}(s)=s^{-1}\left(\sum_{j=0}^{l} s^{j / l} \xi^{j}\right)$, where $\xi^{j} \in \boldsymbol{R}^{n}, \xi^{0} \in \boldsymbol{R}^{n} \backslash\{0\}$ and $l \in N$, and put $\sigma \equiv \sigma(\hat{\eta})=\max _{0 \leqq j \leqq m-1, x \in \boldsymbol{R}^{n}} n_{+}\left(P_{m}, Q_{j}(x, \cdot) ; \hat{\eta}\right) /\left(m-j+n_{+}\left(P_{m}, Q_{j}(x, \cdot) ; \hat{\eta}\right)\right)$.

Now assume that $0<\sigma(<1)$. We can assume without loss of generality that

$$
\sigma=\max _{0 \leqq j \leqq m-1} n_{+}\left(P_{m}, Q_{j}(0, \cdot) ; \hat{\eta}\right) /\left(m-j+n_{+}\left(P_{m}, Q_{j}(0, \cdot) ; \hat{\eta}\right)\right)
$$

and that

$$
n_{+}\left(P_{m}, Q_{j}(0, \cdot) ; \hat{\eta}\right) \geqq n_{+}\left(P_{m}, Q_{j}(0, \cdot) ; \hat{\eta}_{a}^{\lambda}\right)
$$

if $\sigma=n_{+}\left(P_{m}, Q_{j}(0, \cdot) ; \hat{\eta}\right) /\left(m-j+n_{+}\left(P_{m}, Q_{j}(0, \cdot) ; \hat{\eta}\right)\right), 0 \leqq \lambda<1$ and $a \in \boldsymbol{R}$. Moreover we may assume that

$$
n_{+}\left(P_{m}, Q_{j}(x, \cdot) ; \hat{\eta}(s)+\zeta\right) /\left(m-j+n_{+}\left(P_{m}, Q_{j}(x, \cdot) ; \hat{\eta}(s)+\zeta\right)\right) \leqq \sigma
$$

for all $x \in \boldsymbol{R}^{n}, \zeta \in \boldsymbol{R}^{n}$ and $0 \leqq j \leqq m-1$. In fact, these easily follow from Lemma 1.2.4 and its proof. Put $\eta(s)=s^{-\sigma} \hat{\eta}\left(s^{1-\sigma}\right)$. Then we have

$$
\operatorname{order}_{s} P_{m}\left(\eta(s)-i s^{-\sigma} \vartheta\right) \leqq \operatorname{order}_{s} Q_{j}\left(x, \eta(s)-i s^{-\sigma} \zeta\right)
$$

for all $x \in \boldsymbol{R}^{n}, \zeta \in \boldsymbol{R}^{n}$ and $0 \leqq j \leqq m-1$. Thus

$$
\begin{aligned}
& P(x, D)\left(\exp \left[i \gamma^{-1} x \cdot \eta(s)+i s^{-\sigma} \psi(x)\right] v(x)\right) \\
& \quad=\exp \left[i \gamma^{-1} x \cdot \eta(s)+i s^{-\sigma} \psi(x)\right] \sum_{j=0}^{m} \sum_{\alpha} \gamma^{|\alpha|-j} s^{-j+|\alpha|+\nu_{j \alpha}} / \alpha !
\end{aligned}
$$




$$
\begin{array}{r}
\times\left(C_{j \alpha}(x)+o(1)\right) \sum_{\beta \leqq \alpha} \alpha ! /((\alpha-\beta) ! \beta !)\left\{\left(s^{-\sigma} \psi_{x}\right)^{\alpha-\beta}+o\left(s^{-\sigma|\alpha-\beta|}\right)\right\} D^{\beta} v(x) \\
\text { as } s \rightarrow+0
\end{array}
$$

where

$$
\begin{aligned}
& P_{m}^{(\alpha)}(s \eta(s))=s^{\nu_{m \alpha}}\left(C_{m \alpha}+o(1)\right), \quad s \rightarrow+0, \quad C_{m \alpha} \neq 0, \\
& Q_{j}^{(\alpha)}(x, s \eta(s))=s^{\nu j \alpha}\left(C_{j \alpha}(x)+o(1)\right), \quad s \rightarrow+0, \quad C_{j \alpha}(x) \neq 0, \\
& \text { if } Q_{j}^{(\alpha)}(x, s \eta(s)) \neq 0, \quad 0 \leqq j \leqq m-1 \text {. }
\end{aligned}
$$

It follows from (2.2.2) and (2.2.4) that

$$
j-|\alpha|-\nu_{j \alpha}+\sigma|\alpha| \leqq \max _{\beta}\left(m-|\beta|-\nu_{m \beta}+\sigma|\beta|\right) \equiv \nu_{1}, \quad 0 \leqq j \leqq m-1,
$$

and that there are $j$ with $0 \leqq j \leqq m-1$ and a multi-index $\alpha$ such that $j-|\alpha|-$ $\nu_{j \alpha}+\sigma|\alpha|=\nu_{1}$ and $C_{j \alpha}(0) \neq 0$. Therefore we have

$$
\begin{aligned}
& P(x, D)\left(\exp \left[i \gamma^{-1} x \cdot \eta(s)+i s^{-\sigma} \psi(x)\right] v(x)\right) \\
& =s^{-\nu_{1}} \exp \left[i \gamma^{-1} x \cdot \eta(s)+i s^{-\sigma} \psi(x)\right]\left[\sum_{\alpha} 1 / \alpha ! T_{\gamma}^{(\alpha)}\left(x, \psi_{x}\right) s^{\sigma|\alpha|} D^{\alpha} v(x)\right. \\
& \left.+\sum_{\alpha, j \geqq 1} s^{|\alpha|+j \delta} a_{j \alpha}(x) D^{\alpha} v(x)\right],
\end{aligned}
$$

where

$$
T_{r}(x, \xi)=\sum_{j-|\alpha|-\nu_{j \alpha}+\sigma|\alpha|=\nu_{1}} \gamma^{|\alpha|-j} C_{j \alpha}(x) \xi^{\alpha} / \alpha !
$$

and $\delta \in \boldsymbol{Q}, \delta>0$. From the proof of Theorem 1.2.5 there are $x^{0} \in \boldsymbol{R}^{n}$, a neighborhood $U$ of $x^{0}, \eta^{0^{\prime}} \in \boldsymbol{R}^{n-1}$, a neighborhood $V$ of $\eta^{0^{\prime}}$ and $\gamma \in \boldsymbol{R}$ such that $x^{0}$ belongs to a sufficiently small neighborhood of 0 and the equation $T_{r}\left(x, \tau, \eta^{\prime}\right)$ $=0$ in $\tau$ has a root with negative imaginary part for $\left(x, \eta^{\prime}\right) \in U \times V$ which has constant multiplicity $q$ for $\left(x, \eta^{\prime}\right) \in U \times V$. Then there is a complex analytic phase function $\psi(x)$ in $U$ such that

$$
\begin{aligned}
& T_{r}^{(\alpha)}\left(x, \psi_{x}\right)=0, \quad|\alpha|<q, \\
& \left(\partial / \partial \xi_{1}\right)^{q} T_{r}\left(x^{0}, \psi_{x}\left(x^{0}\right)\right) \neq 0, \quad \operatorname{Im} \psi\left(x^{0}\right)=0, \\
& \operatorname{Im} \psi(x) \geqq \varepsilon\left(x_{1}^{0}-x_{1}\right)+\varepsilon\left|x-x^{0}\right|^{2} \quad \text { for } x_{1} \leqq x_{1}^{0}, \\
& \operatorname{Im} \psi(x) \geqq C\left(x_{1}^{0}-x_{1}\right)+\varepsilon\left|x-x^{0}\right|^{2} \quad \text { for } x_{1} \geqq x_{1}^{0},
\end{aligned}
$$

where $\varepsilon>0$. Thus we can write

$$
\begin{aligned}
& P(x, D)\left(\exp \left[i \gamma^{-1} x \cdot \eta(s)+i s^{-\sigma} \psi(x)\right] v(x)\right) \\
& \quad=s^{-\nu_{1}} \exp \left[i \gamma^{-1} x \cdot \eta(s)+i s^{-\sigma} \psi(x)\right] P_{(s)}(x, D) v(x) \quad \text { in } U, \\
& P_{(s)}(x, D)=s^{\sigma q}\left\{Q_{0}(x, s, D)+\sum_{k=1}^{m-p} s^{\sigma k} Q_{k}(x, s, D)\right\},
\end{aligned}
$$

where

$$
\begin{aligned}
Q_{0}(x, s, D)= & \sum_{|\alpha|=q} 1 / \alpha ! T_{\gamma}^{(\alpha)}\left(x, \psi_{x}\right) D^{\alpha} \\
& +\sum_{j \delta+\sigma(|\alpha|-q)<\sigma, j \geqq 1} s^{j \delta+\sigma(|\alpha|-q)} a_{j \alpha}(x) D^{\alpha}
\end{aligned}
$$




$$
\begin{aligned}
Q_{k}(x, s, D)= & \sum_{|\alpha|=q+k} 1 / \alpha ! T_{r}^{(\alpha)}\left(x, \psi_{x}\right) D^{\alpha}+\sum_{|\alpha|=q+k, j \geqq 1} s^{j \delta} a_{j \alpha}(x) D^{\alpha} \\
& +\delta_{k 1} \sum_{|\alpha| \leqq q, \sigma \leqq j \delta+\sigma(|\alpha|-q), j \geqq 1} s^{j \hat{j}+\sigma(|\alpha|-q-1)} a_{j \alpha}(x) D^{\alpha}, \quad 1 \leqq k \leqq m-q .
\end{aligned}
$$

Here $\delta_{k 1}=1$ if $k=1,=0$ if $k \neq 1$. Choose a rational number $\sigma^{\prime}$ so that $\sigma-\delta / q$ $\leqq \sigma^{\prime}<\sigma$ and $\sigma^{\prime}>0$. Then $Q_{0}(x, s, D)$ can be written in the form

$$
Q_{0}(x, s, D) \equiv Q_{0}(x, \bar{\omega} ; D)=\sum_{0 \leqq k, k+|\alpha| \leqq q} b_{k \alpha}(x, s) \bar{\omega}^{k} D^{\alpha},
$$

where $\bar{\omega}=s^{-\sigma^{\prime}}$ and $\left|b_{k \alpha}(x, 0)\right|<\infty$, since $\delta+\sigma(|\alpha|-q) \geqq \sigma^{\prime}(|\alpha|-q)$. It is easy to see that the coefficient of $D_{1}^{q}$ of $Q_{0}(x, \bar{\omega} ; D)$ does not vanish in a neighborhood of $x^{0}$ if $s$ is sufficiently small. Let us consider the equations

$$
\begin{aligned}
& Q_{0}(x, \bar{\omega} ; D) v_{s}^{(n)}=-g_{s}^{(n)}, \\
& \left.v_{s}^{(0)}\right|_{\Omega_{t}}=1,\left.\quad D_{1}^{j} v_{s}^{(0)}\right|_{\Omega_{t}}=0, \quad 1 \leqq j \leqq q-1, \\
& \left.D_{1}^{j} v_{s}^{(n)}\right|_{\Omega_{t}}=0, \quad 0 \leqq j \leqq q-1, \quad n=1,2, \cdots,
\end{aligned}
$$

where $t=x_{1}^{0}, \Omega_{t}=\left\{x \in \Omega ; x_{1}=t\right\}$ and

$$
g_{s}^{(n)}=\sum_{0<k \leqq \min (n, m-q)} s^{\sigma k} Q_{k}(x, s ; D) v_{s}^{(n-k)} .
$$

Define

$$
\Phi_{s}^{(n)}(\tau, \theta ; \omega)=\sum_{\alpha} \tau^{\alpha_{1}} \theta^{\left|\alpha^{\prime}\right|}\left|\alpha ! \sum_{0 \leqq k, k+|\beta| \leqq q-1}\right|\left(D^{\alpha+\beta} v_{s}^{(n)}\right)\left(x^{0}\right) \mid \omega^{k},
$$

where $\alpha^{\prime}=\left(\alpha_{2}, \cdots, \alpha_{n}\right)$ and $\omega \geqq \bar{\omega} \gg 1$.

Lemma 2.2.4 ([11]). There exist positive numbers $A, M, a_{0}$ and $b$ such that

$$
\begin{array}{r}
\Phi_{s}^{(n)}(\tau, \theta ; \omega) \ll A^{n+1} s^{\sigma n} \omega^{q-1} \sum_{k=0}^{2 n} \omega^{n-k} k !(1-\tau / a-\theta / b)^{-k-1} \exp [M \tau \omega], \\
n=0,1,2, \cdots,
\end{array}
$$

for sufficiently large $\omega$, where $\sum_{j, k=0}^{\infty} a_{j k} \tau^{j} \theta^{k} \gg \sum_{j, k=0}^{\infty} b_{j k} \tau^{j} \theta^{k}$ means that $a_{j k} \geqq\left|b_{j k}\right|$ for all $j$ and $k$.

From Lemma 2.2.4 we have

$$
\sum_{|\alpha| \leqq m}\left|D^{\alpha} v_{s}^{(n)} ; K^{\prime \prime}, \kappa, \theta\right| \leqq B^{n+1} s^{\sigma n} \omega^{m} \sum_{k=0}^{2 n} \omega^{n-k} k ! \exp \left[M_{1} \omega\right]
$$

where $K^{\prime \prime}$ is a neighborhood of $x^{0}$ and $B$ and $M_{1}$ are sufficiently large numbers depending on $K^{\prime \prime}$. Thus, if $n \leqq \omega / 4$, then

$$
\sum_{|\alpha| \leqq m}\left|D^{\alpha} v_{s}^{(n)} ; K^{\prime \prime}, \kappa, \theta\right| \leqq 2 B^{n+1} s^{\sigma n} \omega^{m+n} \exp \left[M_{1} \omega\right] .
$$

Put $\omega=4 N, s^{-\sigma}=\omega B e^{L}$ and

$$
V_{s} \equiv V_{s}^{(N)}=\sum_{n=0}^{N} v_{s}^{(n)},
$$

where $L$ will be determined later. Then we have 


$$
\begin{aligned}
& \sum_{|\alpha| \leqq m}\left|D^{\alpha} V_{s} ; K^{\prime \prime}, \kappa, \theta\right| \leqq C N^{m} \exp \left[4 M_{1} N\right], \\
& \left|P_{(s)} V_{s} ; K^{\prime \prime}, \kappa, \theta\right| \leqq C \exp \left[4 M_{1} N-L N\right] .
\end{aligned}
$$

Put

$$
u=u_{(s)}=\exp \left[i \gamma^{-1} x \cdot \eta(s)+i s^{-\sigma} \psi(x)\right] V_{s} \chi,
$$

where $\chi \in \mathscr{D}^{\left\{x_{1}\right\}}\left(\Omega_{T}^{+}\right), 1<\kappa_{1}<\kappa$, supp $\chi$ is contained in a small neighborhood of $x^{0}$ in $K^{\prime \prime}$ and $\chi=1$ in a neighborhood of $x^{0}$. Then we have

$$
\begin{aligned}
\left|u_{(s)} ; K_{t+\tau}^{\prime-}, \kappa, \theta\right| \leqq C\left|V_{s} ; K^{\prime \prime}, \kappa, \theta\right| \mid \exp \left[i \gamma^{-1} x \cdot \eta(s)\right. \\
\\
\left.+i s^{-\sigma} \psi(x)\right], K_{t+\tau}^{\prime \prime}, \kappa, 2 \theta \mid .
\end{aligned}
$$

It follows from (2.2.7) and (2.2.8) and the lemmas in [11] that

$$
\left|u_{(s)} ; K_{t+\tau}^{\prime-}, \kappa, \theta\right| \leqq C N^{m} \exp \left[4 M_{1} N+d\left(s^{-1} \theta\right)^{1 / \kappa}+c \tau s^{-\sigma}\right] .
$$

Therefore, if $\kappa \geqq 1 / \sigma, 0<\tau \leqq \tau(L)$ and $0<\theta \leqq \theta(L)$, then

$$
\left|u_{(s)} ; K_{t+\tau}^{\prime-}, \kappa, \theta\right| \leqq C \exp \left[5 M_{1} N\right]
$$

On the other hand we have

$$
\left|P u_{(s)} ; K_{t+\tau}^{\prime-}, \kappa, \theta\right| \leqq C \exp \left[-6 M_{1} N\right]
$$

if $L$ is fixed sufficiently large, $0<\tau \leqq \tau(L), 0<\theta \leqq \theta(L)$ and $N \geqq N_{0}$. Lemma 2.2.3 and (2.2.9) and (2.2.10) show that the Cauchy problem (2.2.1) is not weakly $\mathscr{E}^{[\kappa\}}$-correct if $\kappa \geqq 1 / \sigma$. Thus we have the following

TheOREM 2.2.5. If the Cauchy problem (2.2.1) is weakly $\mathscr{E}^{\{x\}}$-correct, then $\kappa \leqq \kappa_{0}$.

From Theorem 2.2.5 Theorem 2.2.1 easily follows. We could not prove the existence of $\hat{\eta}(s)$ such that $\sigma(\hat{\eta})=1 / \kappa_{0}$ unless $n \leqq 3$.

\subsection{Necessary and sufficient condition for $\mathscr{E}$-well posedness}

In this section we assume that the coefficients of $Q(x, D)$ are in $C^{\infty}(=\mathscr{E})$. Following Ivrii and Petkov [12], we shall show that the sufficient condition for Cauchy problem (2.1.4) to be $\mathscr{E}$-well posed, which is given by Dunn [3], is also a necessary condition when the Cauchy problem (2.1.4) has the finite propagation property.

Consider the Cauchy problem

$$
\left\{\begin{array}{l}
P(x, D) u(x)=f(x) \quad \text { in } \Omega \cap\left\{x_{1}<T_{+}\right\}, \\
\operatorname{supp} u \subset \bar{G}_{T}^{+}
\end{array}\right.
$$


where $T_{-} \leqq T \leqq T_{+}, \Omega$ is an open domain in $R^{n}$ and $G=\Omega \cap\left\{T_{-} \leqq x_{1} \leqq T_{+}\right\}$. $G_{T}^{+}$if

Definition 2.3.1. The Cauchy problem (2.3.1) is said to be well-posed in

(E) for every $f \in C_{0}^{\infty}$ with $\operatorname{supp} f \subset \bar{\Omega}_{T}^{+}$there exists a solution $u \in \mathscr{D}^{\prime}(\Omega)$ satisfying (2.3.1), and if

(U) $u=0$ in $\Omega_{t}^{-}$for every $t$ with $T<t \leqq T_{+}$, provided that $u \in \mathscr{D}^{\prime}(\Omega)$ satisfies (2.3.1) and that $P u=0$ in $\Omega_{t}^{-}$.

We say that the Cauchy problem (2.3.1) has the finite propagation property if there is a proper convex open cone $\Gamma$ in $\boldsymbol{R}^{n}$ with its vertex at 0 such that $\bar{\Gamma} \subset\left\{x_{1}>0\right\} \cup\{0\}$ and the following statement holds for every $\hat{x} \in G_{T}^{+}$with $\hat{x}_{1}>$ $T$ and $\{\hat{x}-\Gamma\} \cap G_{T}^{+} \subset G_{T}^{+}:$If $u \in \mathscr{D}^{\prime}(\Omega)$, supp $u \subset \bar{G}_{T}^{+}$and $P u=0$ in $\{\hat{x}-\Gamma\} \cap \Omega$, then $u=0$ in $\{\hat{x}-\Gamma\} \cap \Omega$.

LEMma 2.3.2 ([12]). Assume that the Cauchy problem (2.3.1) is well-posed in $G_{T}^{+}$and has the finite propagation property. Let $K$ be a compact set in $\Omega$ and $\hat{x} \in K \cap G$ with $\hat{x}_{1}>T$. Put $\Gamma(\hat{x})=\hat{x}-\Gamma$. Let $\left(1, a_{2}, \cdots, a_{n}\right) \in \Gamma$ and $\delta_{1}>$ $\delta_{2}>\cdots>0$. We define a strictly hyperbolic operator $M_{p}$ for each integer $p \geqq 0$ by

$$
\begin{array}{ll}
M_{p}=\prod_{j=1}^{p / 2}\left(\left(D_{1}+\sum_{k=2}^{n} a_{k} D_{k}\right)^{2}-\delta_{j}^{2} \sum_{k=2}^{n} D_{k}^{2}\right) & \text { if } p \text { is even }, \\
M_{0}=1, M_{p}=\left(D_{1}+\sum_{k=2}^{n} a_{k} D_{k}\right) M_{p-1} & \text { if } p \text { is odd. }
\end{array}
$$

Then there exist non-negative integers $p^{\prime}$ and $q^{\prime}$ and a constant $C$ such that

$$
\|u\|_{C(\Gamma(\hat{x}))} \leqq C\left\|P M_{p^{\prime}} u\right\|_{C^{\prime}(\Gamma(\hat{x}))}
$$

for all $u \in C_{0}^{\infty}\left(K_{T}^{+}\right)$.

Making an asymptotic change of variables $y=s^{-\sigma^{\prime}} x$, it follows from Lemma 2.3.2 that

$$
\|u\|_{C(\Gamma(0))} \leqq C s^{-\nu}\left\|P_{s} M_{p^{\prime}, s} u\right\|_{C q^{\prime}(\Gamma(0))}
$$

for every $u \in C_{0}^{\infty}(W)$ and $0<s \leqq s_{0}(W)$ if $\hat{x}=0$, where $W$ is a compact set in $\boldsymbol{R}^{n}$ and $P_{s}(y, D)=P\left(s^{\sigma^{\prime}} y, s^{-\sigma^{\prime}} D\right), M_{p^{\prime}, s}(y, D)=M_{p^{\prime}}\left(s^{\sigma^{\prime}} y, s^{-\sigma^{\prime}} D\right)$. We apply (2.3.2) to

$$
u_{s}(y)=\sum_{j=0}^{N} s^{-j / \tau} v_{j}(y) E(y, s),
$$

where $E(y, s)=\exp \left[i\left\{s^{-1} l^{0}(y)+\sum_{j=0}^{\mu-1} s^{-(\mu-j) / \tau} l^{j+1}(y)\right\}\right], \mu, \tau \in N, \quad 1 \leqq \mu<\tau, \quad 0 \in W$ and $v_{j}(y) \in C^{\infty}(W)$ with $v_{0}(0) \neq 0$. Assume that

$$
\operatorname{Im}\left(s^{-1} l^{0}(y)+\sum_{j=0}^{\mu-1} s^{-(\mu-j) / \tau} l^{j+1}(y)\right) \geqq-\varepsilon s^{-\delta} y_{1}
$$

for $y \in W_{0}^{-} \cap \Gamma(0)$ and that 


$$
P_{s} M_{p^{\prime}, s} u_{s}=O\left(s^{N_{1}}\right) E(y, s),
$$

where $N_{1}$ tends to $\infty$ as $N \rightarrow \infty$. Let $\phi \in C_{0}^{\infty}(W)$ such tht $\phi=1$ in a neighborhood of 0. Then (2.3.2) is not valid for $\phi u_{s}$. Therefore it suffices to construct $u_{s}(y)$ satisfying (2.3.4) and (2.3.5) in order to prove that the Cauchy problem (2.3.1) is not well-posed in $G_{T}^{+}$when the Cauchy problem has the finite propagation property. In this section we shall prove the following

TheOREm 2.3.3. The Cauchy problem (2.1.4) is $\mathscr{E}$-well posed for every $t \in \boldsymbol{R}$ and has the finite propagation property if and only if $\kappa_{0}=\infty$, that is, $P(x, \xi)$ is hyperbolic with respect to $\vartheta$ for each fixed $x$ in $\boldsymbol{R}^{n}$.

REMARK. The sufficiency of $\mathscr{E}$-well posedness is given in [3]. If the coefficients of $Q(x, D)$ are in $\mathscr{E}^{(k)}$ for some $\kappa<\kappa_{0}$, then the finite propagation property follows from Theorem 2.1.5.

Now assume that $\kappa_{0}<\infty$. Then there is $\hat{\eta}(s)=s^{-1} \sum_{j=0}^{l} s^{j / l} \xi^{j}$ with $\xi^{j} \in \boldsymbol{R}^{n}$, $\xi^{0} \neq 0$ and $l \in N$ such that

$$
\sigma \equiv \max _{0 \leqq j \leqq m-1, x \in R^{n}} n_{+}\left(P_{m}, Q_{j}(x, \cdot) ; \hat{\eta}\right) /\left(m-j+n_{+}\left(P_{m}, Q_{j}(x, \cdot) ; \hat{\eta}\right)\right)>0 .
$$

We can assume without loss of generality that (2.2.2)-(2.2.4) hold. Put $\eta(s)=$ $s^{-\sigma} \hat{\eta}\left(s^{1-\sigma}\right)$. Then we have also (2.2.5). It follows from the proof of Theorem 1.2.5 that $T_{r}\left(0, \tau, \eta^{0^{\prime}}\right)=0$ has a root $\tau_{0}$ with $\operatorname{Im} \tau_{0}<0$ for a fixed $\eta^{0^{\prime}} \in \boldsymbol{R}^{n-1}$ and some $\gamma \in \boldsymbol{R}$. Let $q$ be the multiplicity of the root $\tau_{0}$ and put $\eta_{0}=\left(\tau_{0}, \eta^{0^{\prime}}\right)$ and $\psi(x)=x \cdot \eta^{0}$. Moreover we may assume that $T_{r}^{(\alpha)}\left(0, \eta^{0}\right)=0$ for $|\alpha|<q$, choosing $\eta^{0^{\prime}}$ and $\gamma$ suitably. We make an asymptotic change of variables $y=s^{-\sigma^{\prime}} x$, where $0<\sigma^{\prime}<\sigma$ and $\sigma^{\prime}$ is determined later. Then we have

$$
\begin{aligned}
P_{s}(y, D) & \left(\exp \left[i \gamma^{-1} s^{\sigma^{\prime}} y \cdot\left(\eta(s)+\gamma s^{-\sigma} \eta^{0}\right)\right] v_{s}(y)\right)=s^{-\nu_{1}} \exp \left[i \gamma^{-1} s^{\sigma^{\prime}} y \cdot\left(\eta(s)+\gamma s^{-\sigma} \eta^{0}\right)\right] \\
\times & \left\{\sum_{|\alpha| \geqq q} 1 / \alpha ! s^{\left(\sigma-\sigma^{\prime}|\alpha|\right.} T_{\gamma}^{(\alpha)}\left(0, \eta^{0}\right) D^{\alpha} v_{s}(y)\right. \\
& +\sum_{\alpha} \sum_{1 \leqq|\beta| \leqq N} s^{\left(\sigma-\sigma^{\prime}\right)|\alpha|+\sigma^{\prime}|\beta| \beta \mid} y^{\beta} /(\alpha ! \beta !) T_{\gamma(\beta)}^{(\alpha)}\left(0, \eta^{0}\right) D^{\alpha} v_{s} \\
& \left.+\sum_{\alpha, j \geq 1,|\beta| \leqq N} s^{\left(\sigma-\sigma^{\prime}\right)|\alpha|+j \sigma+\sigma^{\prime}|\beta|} y^{\beta} / \beta ! a_{j \alpha(\beta)}(0) D^{\alpha} v_{s}+s^{\sigma^{\prime} N} R_{N}(y, s, D) v_{s}\right\},
\end{aligned}
$$

where $T_{\gamma(\beta)}^{(\alpha)}(x, \xi)=\left(\partial^{|\alpha|+|\beta|} / \partial \xi^{\alpha} \partial x^{\beta}\right) T_{\gamma}(x, \xi)$ and $R_{N}(y, s, D)$ is a differential operator of order $m$ with smooth coefficients which together with their derivatives are uniformly bounded with respect to $s$ in each domain $K \Subset \boldsymbol{R}^{n}, 0<s \leqq s_{0}(K)$. Here the $a_{j \alpha}(x)$ also depend on $\eta^{0}$. Thus $a_{j \alpha}(x) \equiv a_{j \alpha}\left(x, \eta^{0}\right)$ and $a_{j \alpha(\beta)}(0) \equiv$ $a_{j \alpha(\beta)}\left(0, \eta^{0}\right)$. Choose a rational number $\sigma^{\prime}$ so that $0 \leqq q\left(\sigma-\sigma^{\prime}\right)<\sigma^{\prime}<\sigma$ and $\delta>$ $q\left(\sigma-\sigma^{\prime}\right)$, that is, $\max \{\sigma /(q+1), \sigma-\delta / q\}<\sigma^{\prime}<\sigma$. Then we have

$$
\begin{aligned}
P_{s}(y, D) & \left(\exp \left[i \gamma^{-1} s^{\sigma^{\prime}} y \cdot\left(\eta(s)+\gamma s^{-\sigma} \eta^{0}\right)\right] v_{s}(y)\right) \\
= & s^{-\nu_{1}+q\left(\sigma-\sigma^{\prime}\right)} \exp \left[i \gamma^{-1} s^{\sigma^{\prime}} y \cdot\left(\eta(s)+\gamma s^{-\sigma} \eta^{0}\right)\right] \\
& \times\left\{\sum_{|\alpha|=q} 1 / \alpha ! T_{r}^{(\alpha)}\left(0, \eta^{0}\right) D^{\alpha} v_{s}+\sum_{j=1}^{N_{1}-1} s^{j \sigma^{\prime}} c_{j}(y, D) v_{s}+s^{N_{10^{\prime}}} \hat{R}_{N_{1}}(y, s, D) v_{s}\right\},
\end{aligned}
$$


where $\delta^{\prime}$ is a positive rational number, $c_{j}(y, D)$ is a differential operator of order $m$ with polynomial coefficients of $y$ and $\hat{R}_{N_{1}}(y, s, D)$ has the same properties as $R_{N}(y, s, D)$. In the above argument we may replace $P$ by $P M_{p^{\prime}}$. Let $E(y, s)=\exp \left[i \gamma^{-1} s^{\sigma^{\prime}} y \cdot\left(\eta(s)+\gamma s^{-\sigma} \eta^{0}\right)\right]$. Then we construct an asymptotic solution $u_{s}$ of the form (2.3.3) satisfying (2.3.5). Thus Theorem 2.3.3 easily follows, since $\operatorname{Im}\left\{\gamma^{-1} s^{\sigma^{\prime}} y \cdot\left(\eta(s)+\gamma s^{-\sigma} \eta^{0}\right)\right\}=s^{\sigma^{\prime}-\sigma} y_{1} \operatorname{Im} \tau_{0}$.

\subsection{Wave front sets of solutions}

In this section we assume that the condition (A) is fulfilled. Let $E(x, y)$ be the fundamental solution for $P(x, D)$, that is,

$$
\begin{aligned}
& P\left(x, D_{x}\right) E(x, y)=\delta(x-y), \\
& \text { supp } E \subset\left\{(x, y) \in \boldsymbol{R}^{2 n} ; x-y \in \Gamma\left(P_{m}, \vartheta\right)^{*}\right\} .
\end{aligned}
$$

Define $E_{l}(x, y)$ by

$$
\begin{aligned}
& P_{m}(D) E_{0}(x, y)=\delta(x-y), \\
& P_{m}(D) E_{l+1}(x, y)=-Q(x, D) E_{l}(x, y), \\
& \operatorname{supp} E_{l}(x, y) \subset\left\{(x, y) \in R^{2 n} ; x-y \in \Gamma\left(P_{m}, \vartheta\right)^{*}\right\} \quad l=0,1,2, \cdots
\end{aligned}
$$

Then, repeating the same arguments as in $\S 2.1$, we have

$$
E(x, y)=\sum_{l=0}^{\infty} E_{l}(x, y) \quad \text { in } \mathscr{D}^{\left(\kappa_{0}\right)^{\prime}}\left(R^{2 n}\right) .
$$

Theorem 2.4.1. Under the condition (A) we have

$$
W F_{*_{\kappa_{1}}}(E(x, y)) \subset\left\{((x, y),(\xi,-\xi)) \in T^{*} R^{2 n} \backslash 0 ; x-y \in \Gamma\left(P_{m \xi}, \vartheta\right)^{*}\right\} .
$$

Let $x^{0}, y^{0} \in \boldsymbol{R}^{n}$ and $\xi^{0} \in \boldsymbol{R}^{n} \backslash\{0\}$. Let us first prove that $\left(\left(x^{0}, y^{0}\right),\left(\xi^{0},-\xi^{0}\right)\right) \notin$ $W F_{*_{1}}(E(x, y))$ if $x^{0}-y^{0} \notin \Gamma\left(P_{m \xi^{0}}, \vartheta\right)^{*}$. Assume that $x^{0}-y^{0} \notin \Gamma\left(P_{m \xi 0}, \vartheta\right)^{*}$. Then there are neighborhoods $U_{1}$ and $U_{2}$ of $x^{0}$ and $y^{0}$, respectively, and $\eta^{0} \in \Gamma\left(P_{m \xi^{0}}, \vartheta\right)$ such that

$$
(x-y) \cdot \eta^{0}<0 \quad \text { for all } x \in U_{1} \text { and } y \in U_{2} .
$$

We need the following lemma in order to prove the above statement.

LEMma 2.4.2. Put $\nu=\operatorname{deg} p^{j}$. Then there exist an open convex conic neighborhood $\Gamma$ of $\xi^{0}$ and positive numbers $t_{0}$ and $C$ such that

$$
\left|p^{j}\left(\xi-i\left(t|\xi| \eta^{0}+\gamma(1+|\xi|)^{1 / \alpha_{0}} \vartheta\right)\right) / P_{m}\left(\xi-i\left(t|\xi| \eta^{0}+\gamma(1+|\xi|)^{1 / \kappa_{0}} \vartheta\right)\right)\right| \leqq C \gamma^{\nu-m}
$$

if $\xi \in \Gamma, 0 \leqq t \leqq t_{0}$ and $\gamma \geqq 1$.

REMARK. We can also prove Lemma 1.2 .7 by the same method as used here. 
Proof. Denote by $J$ the left-hand side of (2.4.2). Then we have

$$
J=\left\{\gamma(1+|\xi|)^{1 / \kappa_{0}}\right\}^{\nu-m}\left|p^{j}\left(\hat{\xi}-i\left(t|\hat{\xi}| \eta^{0}+\vartheta\right)\right) / P_{m}\left(\hat{\xi}-i\left(t|\hat{\xi}| \eta^{0}+\vartheta\right)\right)\right|,
$$

where $\hat{\xi}=\left\{\gamma(1+|\xi|)^{1 / x_{0}}\right\}^{-1} \xi$. From Lemma 3.4 in [20] it follows that

$$
\tilde{p}^{j}\left(\hat{\xi}-i\left(t|\hat{\xi}| \eta^{0}+\vartheta\right)\right) \leqq C(1+|\hat{\xi}|)^{n_{\nu}(P)}\left|P_{m}\left(\hat{\xi}-i\left(t|\hat{\xi}| \eta^{0}+\vartheta\right)\right)\right|, \quad \xi \in \Gamma,
$$

if $t|\hat{\xi}| \leqq \rho$, where a positive number $\rho$ is fixed and $\Gamma$ is a suitable conic neighborhood of $\xi^{0}$. Moreover we have

$$
\begin{aligned}
\tilde{p}^{j}\left(t^{-1}|\hat{\xi}|^{-1} \hat{\xi}-i\left(\eta^{0}+t^{-1}|\hat{\xi}|^{-1} \vartheta\right)\right) \leqq C\left(1+t^{-1}|\hat{\xi}|^{-1}|\hat{\xi}|\right)^{n_{\nu}(P)} \\
\\
\quad \times\left|P_{m}\left(t^{-1}|\hat{\xi}|^{-1} \hat{\xi}-i\left(\eta^{0}+t^{-1}|\hat{\xi}|^{-1} \vartheta\right)\right)\right|, \quad \xi \in \Gamma,
\end{aligned}
$$

if $t|\hat{\xi}| \geqq \rho . \quad$ Thus we have

$$
\left|p^{j}\left(\hat{\xi}-i\left(t|\hat{\xi}| \eta^{0}+\vartheta\right)\right)\right| \leqq C(1+|\hat{\xi}|)^{n_{\nu}(P)}\left|P_{m}\left(\hat{\xi}-i\left(t|\hat{\xi}| \eta^{0}+\vartheta\right)\right)\right|, \quad \xi \in \Gamma .
$$

Since $n_{\nu}(P)-\left(n_{\nu}(P)+m-\nu\right) / \kappa_{0} \leqq 0,(2.4 .2)$ follows from (2.4.3) and (2.4.4).

Q.E.D.

Proof of Theorem 2.4.1. We may assume that the coefficients of $Q(x, D)$ are in $\mathscr{D}^{*_{1}}$. Let $\phi_{j} \in \mathscr{D}^{\left(\kappa_{1}\right)}\left(U_{j}\right), j=1,2$. We have

$$
\begin{aligned}
\mathscr{F}_{(x, y)} & {\left[\phi_{1}(x) \phi_{2}(y) E_{l}(x, y)\right](\xi, \eta)=\sum_{j_{1}, \cdots, j_{l}}(-1)^{l}(2 \pi)^{-(1+l) n} } \\
\times & \int d \zeta^{0} \hat{\phi}_{1}\left(1 / 2 \xi-\zeta^{0}\right) P_{m}\left(1 / 2 \xi+\zeta^{0}\right)^{-1} \\
\times & \left\{\int d \zeta^{1} \hat{q}_{j_{1}}\left(\zeta^{1}\right) p^{j_{1}}\left(1 / 2 \xi+\zeta^{0}-\zeta^{1}\right) P_{m}\left(1 / 2 \xi+\zeta^{0}-\zeta^{1}\right)^{-1}\right. \\
\quad \times & \left\{\int d \zeta^{2} \cdots\right. \\
\times & \left\{\int d \zeta^{l} \hat{q}_{j_{l}}\left(\zeta^{l}\right) p^{j_{l}}\left(1 / 2 \xi+\zeta^{0}-\zeta^{1}-\cdots-\zeta^{l}\right)\right. \\
& \left.\left.\times P_{m}\left(1 / 2 \xi+\zeta^{0}-\zeta^{1}-\cdots-\zeta^{l}\right)^{-1} \hat{\phi}_{2}\left(1 / 2 \xi+\eta+\zeta^{0}-\zeta^{1}-\cdots-\zeta^{l}\right)\right\} \cdots\right\}, \\
\quad l=0,1,2, \cdots, & \quad l=0
\end{aligned}
$$

where $\zeta^{0}=-1 / 2 \xi+w^{0}-i \gamma\left(1+\left|w^{0}\right|\right)^{1 / \kappa_{0}} \vartheta, \zeta^{j}=w^{j}+i a\left(1+\left|w^{j}\right|\right)^{1 / \kappa_{1}} \vartheta, 1 \leqq j \leqq l, w^{j} \in \boldsymbol{R}^{n}$, $0 \leqq j \leqq l$, and $a>0$. Write

$$
\begin{aligned}
\mathscr{F}_{(x, y)}\left[\phi_{1} \phi_{2} E_{l}\right](\xi, \eta)= & \sum_{j_{1}, \cdots, j_{l}}(-1)^{l}(2 \pi)^{-(1+l) n} \\
& \times\left\{\int_{\left|w_{1}\right|+\cdots+\left|w^{l}\right|<\varepsilon|\xi|}+\int_{\left|w^{1}\right|+\cdots+\left|w^{l}\right|>\varepsilon|\xi|}\right\} d \zeta^{0} \cdots d \zeta^{l} \\
\equiv & I_{1}+I_{2} .
\end{aligned}
$$

We first estimate $\left|I_{1}\right|$. Let $\Gamma^{1}$ and $\Gamma^{2}$ be convex conic neighborhoods of $\xi^{0}$ such 
that $\Gamma^{1} \Subset \Gamma^{2} \Subset \Gamma$ and $1 / 2 \xi+\zeta \in \Gamma$ for $\xi \in \Gamma^{2}$ and $\zeta \in \boldsymbol{R}^{n}$ with $|\zeta|<\varepsilon|\xi|$, where $\varepsilon>0$ is chosen suitably. Denote by $V_{t}, 0 \leqq t \leqq t_{0}$, the chain $\left\{\zeta_{t}^{0} \in C^{n} ; \zeta_{t}^{0}=-1 / 2 \xi+w^{0}\right.$ $\left.-i\left(t\left|-1 / 2 \xi+w^{0}\right| \Phi\left(-1 / 2 \xi+w^{0}\right) \eta^{0}+\gamma\left(1+\left|w^{0}\right|\right)^{1 / x_{0}} \vartheta\right), w^{0} \in \boldsymbol{R}^{n}\right\}$, where $\Phi(\zeta) \in C^{\infty}\left(\boldsymbol{R}^{n}\right)$ is positively homogeneous of degree 0 in $|\zeta| \geqq 1$ and $\Phi(\zeta)=1$ on a neighborhood of $\Gamma^{1} \cap\{|\zeta| \geqq 1\}$, supp $\Phi(\zeta) \subset \Gamma^{2} \cap\{|\zeta| \geqq 1 / 2\}, 0 \leqq \Phi(\zeta) \leqq 1$. It follows from Proposition 1.3.1. and (2.4.1) that

$$
\begin{aligned}
\left|\hat{\phi}_{1}\left(1 / 2 \xi-\zeta_{t}^{0}\right) \hat{\phi}_{2}\left(\eta+1 / 2 \xi+\zeta_{t}^{0}-\zeta^{1}-\cdots-\zeta^{l}\right)\right| \\
\leqq C_{1} \exp \left[A\left(\gamma\left(1+\left|w^{0}\right|\right)^{1 / \kappa_{0}}+a \sum_{j=1}^{l}\left(1+\left|w^{j}\right|\right)^{1 / \kappa_{1}}\right)\right. \\
-c t\left|-1 / 2 \xi+w^{0}\right| \Phi\left(-1 / 2 \xi+w^{0}\right)-B_{1}\left|\xi-w^{0}\right|^{1 / \kappa_{1}} \\
\left.-B_{1}\left|\eta+w^{0}-w^{1}-\cdots-w^{l}\right|^{1 / \kappa_{1}}\right]
\end{aligned}
$$

for every $B_{1}>0$ and some $C_{1}$ depending on $B_{1}$, where

$$
\zeta_{t}^{0}=-1 / 2 \xi+w^{0}-i\left(t\left|-1 / 2 \xi+w^{0}\right| \Phi\left(-1 / 2 \xi+w^{0}\right) \eta^{0}+\gamma\left(1+\left|w^{0}\right|^{1 / \kappa_{0}} \vartheta\right)\right.
$$

and $A$ and $c$ are positive constants. Moreover we have

$$
\left|\hat{q}_{j}(\zeta)\right| \leqq C_{2} \exp \left[A|\operatorname{Im} \zeta|-B_{2}|\operatorname{Re} \zeta|^{1 / \kappa 1}\right],
$$

where (2.4.7) holds for every $B_{2}>0$ and some $C_{2}$ depending on $B_{2}$ if $*_{\kappa_{1}}=\left(\kappa_{1}\right)$, and for some positive constants $B_{2}$ and $C_{2}$ if $*_{\kappa_{1}}=\left\{\kappa_{1}\right\}$. Let $\xi \in \Gamma^{1}$. Then we have

$$
\left|p^{j_{k}}\left(1 / 2 \xi+\zeta_{t}^{0}-\zeta^{1}-\cdots-\zeta^{k}\right) / P_{m}\left(1 / 2 \xi+\zeta_{t}^{0}-\zeta^{1}-\cdots-\zeta^{k}\right)\right| \leqq C \gamma^{-\mu}
$$

if $\left|w^{1}\right|+\cdots+\left|w^{k}\right|<\varepsilon|\xi|, \gamma \geqq 1$ and $0 \leqq t \leqq t_{0}$, where $\mu$ is defined by (2.1.5). In fact, (2.4.8) is proved in the proof of Lemma 2.1.2 if $\Phi\left(-1 / 2 \xi+w^{0}\right)=0$. On the other hand we have $-1 / 2 \xi+w^{0}, w^{0} \in \Gamma^{2}$ and $\left|w^{0}\right| \geqq|\xi| / 2$ if $\Phi\left(-1 / 2 \xi+w^{0}\right) \neq 0$ and $\Gamma^{2}$ is sufficiently small. Thus we have

$$
\gamma\left(1+\left|w^{0}\right|\right)^{1 / \kappa_{0}} \geqq \gamma / 2\left(1+\left|w^{0}-w^{1}-\cdots-w^{k}\right|\right)^{1 / \kappa_{0}}
$$

if $\Phi\left(-1 / 2 \xi+w^{0}\right) \neq 0,\left|w^{1}\right|+\cdots+\left|w^{k}\right|<\varepsilon|\xi|$ and $\varepsilon$ is sufficiently small. Then Lemma 2.4.2 gives (2.4.8). From Stokes' formula and (2.4.6)-(2.4.8) it follows that

$$
\begin{aligned}
& \left|I_{1}\right| \leqq\left|\int d \zeta^{0} \int_{|w 1|+\cdots+|w|^{2}<\varepsilon|\xi|} d w^{1} \cdots d w^{l} \cdots\right| \\
& \leqq C C_{1}\left(C C_{2 \gamma^{-\mu}}\right)^{l+1} \int d w^{0} \cdots d w^{l} \\
& \times \exp \left[A\left(\gamma\left(1+\left|w^{0}\right|\right)^{1 / \kappa_{0}}+2 a \sum_{j=1}^{l}\left(1+\left|w^{j}\right|\right)^{1 / \kappa_{1}}\right)\right. \\
& -\delta B_{1}|\xi|^{1 / \kappa_{1}}-\delta B_{1}\left|w^{0}\right|^{1 / \kappa_{1}}-B_{1}\left|\eta+w^{0}-w^{1}-\cdots-w^{l}\right|^{1 / \kappa_{1}} \\
& \left.-B_{2}\left(\left|w^{1}\right|^{1 / \kappa_{1}}+\cdots+\left|w^{l}\right|^{1 / \kappa_{1}}\right)\right], \quad \zeta^{0} \in V_{t_{0}}, \quad \xi \in \Gamma^{1}, \quad \gamma \geqq 1 .
\end{aligned}
$$


Here we have used the inequality

$$
\begin{gathered}
\exp \left[-c t_{0}\left|-1 / 2 \xi+w^{0}\right| \Phi\left(-1 / 2 \xi+w^{0}\right)-B_{1}\left|\xi-w^{0}\right|^{1 / \kappa_{1}}\right] \\
\leqq C \exp \left[-\delta B_{1}|\xi|^{1 / \kappa_{1}}-\delta B_{1}\left|w^{0}\right|^{1 / \kappa_{1}}\right], \quad \xi \in \Gamma^{1},
\end{gathered}
$$

for some $\delta>0$. Choose $a>0$ so that $2 a A \leqq B_{2} / 2$. Then by (2.4.9) we obtain

$$
\left|I_{1}\right| \leqq C_{\gamma} C_{1}\left(C C_{2} \gamma^{-\mu}\right)^{l+1} \exp \left[-\delta B_{1}|\xi|^{1 / \kappa_{1}}\right], \quad \xi \in \Gamma^{1}, \quad \gamma \geqq 1,
$$

since $\exp \left[A \gamma\left(1+\left|w^{0}\right|\right)^{1 / \kappa_{0}}-\delta B_{1}\left|w^{0}\right|^{1 / \kappa_{1}} / 2\right] \leqq C_{r} . \quad\left|I_{2}\right|$ can be estimated without a change of the integral chain. We have

$$
\begin{aligned}
\left|I_{2}\right| \leqq & C C_{1}\left(C C_{2} \gamma^{-\mu}\right)^{l+1} \int_{\left|w^{1}\right|+\cdots+\left|w^{l}\right|>\varepsilon|\xi|} d w^{0} \cdots d w^{l} \\
\times \exp [ & A\left(\gamma\left(1+\mid w^{0}\right)^{1 / \kappa_{0}}+2 a \sum_{j=1}^{l}\left(1+\left|w^{j}\right|\right)^{1 / \kappa_{1}}\right) \\
& -B_{1}\left|\xi-w^{0}\right|^{1 / \kappa_{1}}-B_{1}\left|\eta+w^{0}-w^{1}-\cdots-w^{l}\right|^{1 / \kappa_{1}} \\
& \left.-B_{2}\left(\left|w^{1}\right|^{1 / \kappa_{1}}+\cdots+\left|w^{l}\right|^{1 / \kappa_{1}}\right)\right] .
\end{aligned}
$$

Choose $a>0$ so that $2 a A \leqq B_{2} / 4$. Then we obtain

$$
\left|I_{2}\right| \leqq C_{\gamma} C_{1}\left(C C_{2} \gamma^{-\mu}\right)^{l+1} \exp \left[-\varepsilon B_{2}|\xi|^{1 / \kappa_{1}} / 4\right], \quad \gamma \geqq 1 .
$$

Here we have used the inequality

$$
\exp \left[A \gamma\left(1+\left|w^{0}\right|\right)^{1 / \kappa_{0}}-B_{1}\left|\xi-w^{0}\right|^{1 / \kappa_{1}} / 2-\varepsilon B_{2}|\xi|^{1 / \kappa_{1}} / 4\right] \leqq C_{r^{\circ}}
$$

(2.4.10) and (2.4.11) mean that $\left(\left(x^{0}, y^{0}\right),\left(\xi^{0}, \eta^{1}\right)\right) \notin W F_{*_{1}}(E(x, y))$ if $x^{0}-y^{0} \notin$ $\Gamma\left(P_{m \xi^{0}}, \vartheta\right)^{*}$. Finally let us prove that

$$
\left(\left(x^{0}, y^{0}\right),\left(\xi^{0}, \eta^{1}\right)\right) \notin W F_{*_{1}}(E(x, y)) \quad \text { if } \xi^{0} \neq-\eta^{1} .
$$

Let $\tilde{\Gamma}$ be a conic neighborhood of $\left(\xi^{0}, \eta^{1}\right)$ in $R^{2 n} \backslash\{0\}$. Then we may assume that $|\xi+\eta| \geqq 2 \varepsilon|(\xi, \eta)|, \varepsilon>0$ if $(\xi, \eta) \in \tilde{\Gamma}$. Write

$$
\begin{aligned}
\mathscr{F}_{(x, y)} & {\left[\phi_{1}(x) \phi_{2}(y) E_{l}(x, y)\right](\xi, \eta)=\sum_{j_{1}, \cdots, j_{l}}(-1)^{l}(2 \pi)^{-(1+l) n} } \\
\times & \times \int d \zeta^{0} \hat{\phi}_{1}\left(\xi-\zeta^{0}\right) P_{m}\left(\zeta^{0}\right)^{-1} \\
\times & \left\{\int d \zeta^{1} \hat{q}_{j_{1}}\left(\zeta^{1}\right) p^{j_{1}}\left(\zeta^{0}-\zeta^{1}\right) P_{m}\left(\zeta^{0}-\zeta^{1}\right)^{-1}\right. \\
\times & \left\{\int d \zeta^{2} \cdots\right. \\
\times & \left\{\int d \zeta^{l} \hat{q}_{j_{l}}\left(\zeta^{l}\right) p^{j_{l}}\left(\zeta^{0}-\zeta^{1}-\cdots-\zeta^{l}\right) P_{m}\left(\zeta^{0}-\zeta^{1}-\cdots-\zeta^{l}\right)^{-1}\right. \\
& \left.\left.\times \hat{\phi}_{2}\left(\eta+\zeta^{0}-\zeta^{1}-\cdots-\zeta^{l}\right)\right\} \cdots\right\},
\end{aligned}
$$


where $\zeta^{0}=w^{0}-i \gamma\left(1+\left|w^{0}\right|\right)^{1 / \kappa_{0}} \vartheta, \zeta^{j}=w^{j}+i a\left(1+\left|w^{j}\right|\right)^{1 / \kappa_{1}} \vartheta, 1 \leqq j \leqq l$, and $w^{j} \in \boldsymbol{R}^{n}, 0 \leqq$ $j \leqq l$. Thus (2.1.8) and Proposition 1.3.1 give

$$
\begin{aligned}
\left|\mathscr{F}\left[\phi_{1} \phi_{2} E_{l}\right](\xi, \eta)\right| \leqq & C C_{1}\left(C C_{2} \gamma^{-\mu}\right)^{l+1} \int d w^{0} \cdots d w^{l} \\
\times \exp [ & A\left(\gamma\left(1+\left|w^{0}\right|\right)^{1 / \kappa_{0}}+2 a \sum_{j=1}^{l}\left(1+\left|w^{j}\right|\right)^{1 / \kappa_{1}}\right) \\
& -B_{1}\left|\xi-w^{0}\right|^{1 / \kappa_{1}}-B_{1}\left|\eta+w^{0}-w^{1}-\cdots-w^{l}\right|^{1 / \kappa_{1}} \\
& \left.-B_{2}\left(\left|w^{1}\right|^{1 / \kappa_{1}}+\cdots+\left|w^{l}\right|^{1 / \kappa_{1}}\right)\right], \quad \gamma \geqq 1 .
\end{aligned}
$$

Choose positive numbers $a$ and $B_{1}$ so that $B_{1}=B_{2} / 2$ and $2 a A=B_{2} / 4$. Since

$$
\begin{gathered}
\left|\xi-w^{0}\right|^{1 / \kappa_{1}}+\left|\eta+w^{0}-w^{1}-\cdots-w^{l}\right|^{1 / \kappa_{1}}+\left(\left|w^{1}\right|^{1 / \kappa_{1}}+\cdots+\left|w^{l}\right|^{1 / \kappa_{1}}\right) \\
\geqq 2 \varepsilon^{\prime}|(\xi, \eta)|^{1 / \kappa_{1}}, \varepsilon^{\prime}>0, \quad \text { for }(\xi, \eta) \in \tilde{\Gamma}, \\
\exp \left[A \gamma\left(1+\left|w^{0}\right|\right)^{1 / \kappa_{0}}-B_{1}\left|\xi-w^{0}\right|^{1 / \kappa_{1}} / 2-\varepsilon^{\prime}|\xi|^{1 / \kappa_{1}} / 2\right] \leqq C_{r},
\end{gathered}
$$

we have

$$
\left|\mathscr{F}\left[\phi_{1} \phi_{2} E_{l}\right](\xi, \eta)\right| \leqq C_{\gamma} C_{1}\left(C C_{2} \gamma^{-\mu}\right)^{l+1} \exp \left[-\varepsilon^{\prime} B_{2}|(\xi, \eta)|^{1 / \kappa_{1}} / 4\right]
$$

if $(\xi, \eta) \in \tilde{\Gamma}$ and $\gamma \geqq 1$. This proves (2.4.12). The proof of the theorem is complete.

Q.E.D.

TheOREm 2.4.3. Under the condition (A) we have $\left(\left(x^{0}, y^{0}\right),\left(\xi^{0},-\xi^{0}\right)\right) \notin$ $W F^{\left(\kappa\left(\xi^{0}\right)\right.}(E(x, y))$ for $x^{0}, y^{0} \in \boldsymbol{R}^{n}$ and $\xi^{0} \in \boldsymbol{R}^{n} \backslash\{0\}$, where $\kappa\left(\xi^{0}\right)$ is defined by (2.1.2).

Proof. Let $U_{1}$ and $U_{2}$ be neighborhoods of $x^{0}$ and $y^{0}$, respectively, and let $\phi_{j} \in \mathscr{D}^{(\kappa)}\left(U_{j}\right), j=1,2, \kappa_{1}<\kappa<\kappa_{0}$. We may assume that the coefficients of $Q(x, D)$ are in $\mathscr{D}^{*_{x_{1}}}$. Therefore they are in $\mathscr{D}^{\left({ }^{(x)}\right.}$. Then we have (2.4.13) with $\zeta^{0}=w^{0}-i \gamma\left(1+\left|w^{0}\right|\right)^{1 / \kappa_{0}} \vartheta, \zeta^{j}=w^{j}+i\left(1+\left|w^{j}\right|\right)^{1 / \kappa} \vartheta, 1 \leqq j \leqq l$, and $w^{j} \in \boldsymbol{R}^{n}, 0 \leqq j \leqq l$. Let $\Gamma^{j}, j=0,1,2$, be conic neighborhoods of $\xi^{0}$ such that $\Gamma^{0} \Subset \Gamma^{1} \Subset \Gamma^{2}$ and $\xi+$ $\zeta \in \Gamma^{2}$ if $\xi \in \Gamma^{1}$ and $|\zeta| \leqq \varepsilon|\xi|$, where $\varepsilon>0$ is sufficiently small. Choose $\Phi(\zeta) \in$ $C^{\infty}\left(\boldsymbol{R}^{n}\right)$ so that $\Phi(\zeta)$ is positively homogeneous of degree 0 in $|\zeta| \geqq 1$, supp $\Phi(\zeta) \subset$ $\Gamma^{1} \cap\{|\zeta| \geqq 1 / 2\}, \Phi(\zeta)=1$ on a neighborhood of $\Gamma^{0} \cap\{|\zeta| \geqq 1\}$ and $0 \leqq \Phi(\zeta) \leqq 1$. Put

$$
\zeta_{t}^{0}=w^{0}-i \gamma\left\{\left(1+\left|w^{0}\right|\right)^{1 / \kappa_{0}}+t \Phi\left(w^{0}\right)\left(\left(1+\left|w^{0}\right|\right)^{1 / \kappa(\xi 0)}-\left(1+\left|w^{0}\right|\right)^{1 / \kappa_{0}}\right)\right\} \vartheta, \quad 0 \leqq t \leqq 1 .
$$

Let us first prove the inequality

$$
J \equiv\left|p^{j}\left(\zeta_{t}^{0}-\zeta^{1}-\cdots-\zeta^{k}\right) / P_{m}\left(\zeta_{t}^{0}-\zeta^{1}-\cdots-\zeta^{k}\right)\right| \leqq C \gamma^{-\mu}, \quad \gamma \geqq 1,
$$

where $\mu$ is defined by (2.1.5) with $\kappa_{1}$ replaced by $\kappa$. (2.4.14) follows from (2.1.8) if $w^{0} \oplus \Gamma^{1}$. Let $w^{0} \in \Gamma^{1}$. Then

$$
J \leqq C\left\{\gamma\left(1+\left|w^{0}\right|\right)^{1 / \kappa(\xi 0)}\right\}^{\nu-m}\left(1+\left\{\gamma\left(1+\left|w^{0}\right|\right)^{1 / \kappa(\xi)}\right\}^{-n_{\nu}(\xi 0)}\left|w^{0}\right|^{n_{\nu}\left(\xi^{0}\right)}\right)
$$

if $\left|w^{1}\right|+\cdots+\left|w^{k}\right| \leqq \varepsilon\left|w^{0}\right|$ and $\Gamma^{2}$ and $\varepsilon$ are sufficiently small, where $\nu=\operatorname{deg} p^{j}$ and $n_{\nu}\left(\xi^{0}\right)$ is defined by (2.1.1). Thus we have 


$$
J \leqq C \gamma^{\nu-m} \quad \text { if } \gamma \geqq 1 \quad \text { and } \quad\left|w^{1}\right|+\cdots+\left|w^{k}\right| \leqq \varepsilon\left|w^{0}\right|
$$

Moreover we have

$$
\begin{aligned}
J \leqq & C\left\{\gamma\left(1+\left|w^{0}\right|\right)^{1 / \kappa\left(\xi^{0}\right)}+\sum_{h=1}^{k}\left(1+\left|w^{h}\right|\right)^{1 / \kappa}\right\}^{\nu-m} \\
& \times\left(1+\left\{\gamma\left(1+\left|w^{0}\right|\right)^{1 / \kappa(\xi 0)}+\sum_{h=1}^{k}\left(1+\left|w^{h}\right|\right)^{1 / \kappa}\right\}^{-n_{\nu}(P)}\left\{\sum_{j=1}^{k}\left(1+\left|w^{h}\right|\right)\right\}^{n_{\nu}(P)}\right. \\
\leqq & C \gamma^{-\mu} \quad \text { if } \gamma \geqq 1 \text { and }\left|w^{1}\right|+\cdots+\left|w^{k}\right| \geqq \varepsilon\left|w^{0}\right| .
\end{aligned}
$$

Let $\tilde{\Gamma}$ be a conic neighborhood of $\left(\xi^{0},-\xi^{0}\right)$ in $\boldsymbol{R}^{2 n} \backslash\{0\}$ such that $\{\xi ;(\xi, \eta) \in \tilde{\Gamma}$ for some $\eta\} \Subset \Gamma^{0}$. From Proposition 1.3.1 and (2.4.14) it follows that

$$
\begin{gathered}
\left|\mathscr{F}\left[\phi_{1} \phi_{2} E_{l}\right](\xi, \eta)\right| \leqq\left|\int_{V_{1}} d \zeta^{0} d w^{1} \cdots d w^{l} \cdots\right| \leqq C\left(C C_{B} \gamma^{-\mu}\right)^{l+1} \int d w^{0} \cdots d w^{l} \\
\times \exp \left[A \left\{\gamma\left(1+\left|w^{0}\right|\right)^{1 / \kappa_{0}}+\gamma \Phi\left(w^{0}\right)\left(\left(1+\left|w^{0}\right|\right)^{1 / \kappa(\xi) 0}-\left(1+\left|w^{0}\right|\right)^{1 / \kappa_{1}}\right)\right.\right. \\
\left.+\sum_{j=1}^{l}\left(1+\left|w^{j}\right|\right)^{1 / \kappa}\right\}-B\left|\xi-w^{0}\right|^{1 / \kappa}-B \sum_{j=1}^{l}\left|w^{j}\right|^{1 / \kappa} \\
\left.-B\left|\eta+w^{0}-w^{1}-\cdots-w^{l}\right|^{1 / \kappa}\right], \quad \gamma \geqq 1,
\end{gathered}
$$

where $V_{t}=\left\{\zeta_{t}^{0} ; w^{0} \in \boldsymbol{R}^{n}\right\}, 0 \leqq t \leqq 1$. It is easy to see that

$$
\begin{aligned}
& \left(1+\left|w^{0}\right|\right)^{1 / \kappa(\xi)} \leqq(1+|\xi|)^{1 / \kappa(\xi 0)}+\left(1+\left|\xi-w^{0}\right|\right)^{1 / \kappa(\xi)}, \\
& \exp \left[\gamma\left(1+\left|\xi-w^{0}\right|\right)^{1 / \kappa(\xi)}-\left|\xi-w^{0}\right|^{1 / \kappa}\right] \leqq C_{\gamma} .
\end{aligned}
$$

If $\Phi\left(w^{0}\right) \neq 1$, then we have

$$
1+\left|\xi-w^{0}\right|^{1 / \kappa} \geqq \delta\left(|\xi|^{1 / \kappa}+\left|w^{0}\right|^{1 / \kappa}\right), \quad(\xi, \eta) \in \tilde{\Gamma},
$$

for some $\delta>0$. Thus

$$
\begin{gathered}
\gamma\left\{\left(1+\left|w^{0}\right|\right)^{1 / \kappa_{0}}+\Phi\left(w^{0}\right)\left(\left(1+\left|w^{0}\right|\right)^{1 / \kappa\left(\xi^{0}\right)}-\left(1+\left|w^{0}\right|\right)^{1 / \kappa_{0}}\right)\right\} \\
-\left|\xi-w^{0}\right|^{1 / \kappa} \leqq \gamma\left(1+\left|w^{0}\right|\right)^{1 / \kappa_{0}}-\delta|\xi|^{1 / \kappa}-\delta\left|w^{0}\right|^{1 / \kappa}
\end{gathered}
$$

if $\Phi\left(w^{0}\right) \neq 1$ and $(\xi, \eta) \in \tilde{\Gamma}$. It follows from (2.4.16) with $B=A+1$ and (2.4.17)(2.4.20) that

$$
\left|\mathscr{F}\left[\phi_{1} \phi_{2} E_{l}\right](\xi, \eta)\right| \leqq C_{\gamma}\left(C_{\gamma^{-\mu}}\right)^{l+1} \exp \left[A \gamma|\xi|^{1 / \kappa(\xi \circ)}\right], \quad \gamma \geqq 1,
$$

which proves the theorem.

Q.E.D.

Theorems 2.4.2 and 2.4.3 together with Lemma 1.3.11 give the following

Theorem 2.4.4. Assume that the condition (A) is satisfied and $f \in \mathscr{D}^{\left({ }^{\left(k_{1}\right.}\right)^{\prime}}$ (resp. $\mathscr{D}^{\left\{\kappa_{1}\right\}^{\prime}}$ ) with supp $f \subset\left\{x \in \boldsymbol{R}^{n} ; x_{1} \geqq t\right\}, t \in \boldsymbol{R}$. Let $u$ be the solution of (2.1.4) and $\kappa_{1} \leqq \kappa \leqq \infty$. Then Theorem 1.4.4 is valid if $E * f$ and $\delta(\xi)$ are replaced by $u$ and $1 / \kappa(\xi)$, respectively.

The finite set $\left\{\kappa(\xi) ; \xi \in \boldsymbol{R}^{n} \backslash\{0\}\right\}$ can be written in the form 


$$
\left\{\kappa(\xi) ; \xi \in \boldsymbol{R}^{n} \backslash\{0\}\right\}=\left\{s_{j} ; 1 \leqq j \leqq l\right\}, \quad 1<\kappa_{0}=s_{1}<s_{2}<\cdots<s_{l}=\infty .
$$

Define

$$
\begin{aligned}
\tilde{K}_{j} & =\left\{((x, \xi),(y, \xi)) ; x-y \in \Gamma\left(P_{m \xi}, \vartheta\right)^{*}, \xi \in R^{n} \backslash\{0\} \text { and } \kappa(\xi)=s_{j}\right\}, \quad 1 \leqq j \leqq l, \\
K & =\bigcup_{j=1}^{l} \tilde{K}_{j} .
\end{aligned}
$$

CoRollary 2.4.5. Corollary 1.4.5 is valid if $E * f$ and the $K_{j}$ are replaced by the solution $u$ and the $K_{j}$, respectively.

Corollary 2.4.6. Corollary 1.4.6 is valid if $E * f$ and $\delta(\xi)$ are replaced by the solution $u$ and $1 / \kappa(\xi)$, respectively.

\section{Appendix}

\section{$\S$ A.1. Procedure to determine the class of hyperbolicity in the case $\mathbf{n} \leqq 3$}

Let $H(\xi)$ be a hyperbolic polynomial with respect to $\vartheta$, homogeneous of degree $m$ and let $S(\xi)$ be a homogeneous polynomial of degree $m^{\prime}, m^{\prime}<m$.

Lemma A.1.1. Let $n=2$ and $\xi^{0} \in \boldsymbol{R}^{2} \backslash\{0\}$. Then we have

$$
n\left(H, S ; \xi^{0}\right)= \begin{cases}m^{\prime}-m+\operatorname{deg} H_{\xi^{0}}-\operatorname{deg} S_{\xi^{0}} & \text { if } \operatorname{deg} H_{\xi^{0}} \geqq \operatorname{deg} S_{\xi^{0}}, \\ m^{\prime}-m, & \text { otherwise. }\end{cases}
$$

This lemma is obvious and we shall calculate $n\left(H, S ; \xi^{0}\right)$ when $n=3$.

Lemma A.1.2. Let $Q(\tau, s)$ be a polynomial. Write

$$
\begin{aligned}
& Q(\tau, s)=\tau^{\alpha_{1}} s^{\alpha_{2}} \hat{Q}(\tau, s), \quad \hat{Q}(\tau, 0) \neq 0, \quad \hat{Q}(0, s) \neq 0 \\
& \hat{Q}(\tau, s)=\hat{q}_{0}(s) \tau^{\alpha_{3}}+\hat{q}_{1}(s) \tau^{\alpha_{3}-1}+\cdots+\hat{q}_{\alpha_{3}}(s),
\end{aligned}
$$

where $Q(0, s)=\hat{q}_{\alpha_{3}}(s)$. Moreover write

$$
\begin{aligned}
\hat{Q}(0, s) & =s^{\alpha_{4}} q(s), & & q(0) \neq 0, \\
\hat{q}_{0}(s) & =s^{\alpha_{5}} q_{0}(s), & & q_{0}(0) \neq 0, \\
\hat{Q}(\tau, 0) & =\tau^{\alpha_{6}} q_{1}(\tau), & & q_{1}(0) \neq 0 .
\end{aligned}
$$

Let $\lambda_{j}(s), 1 \leqq j \leqq t$, be the roots of $Q(\tau, s)=0$. Then we have $t=\alpha_{1}+\alpha_{3}$. Put $\nu_{j}$ $=\operatorname{order} \lambda_{j}(s)$. We have

$$
\begin{aligned}
& \sum_{\nu_{j}<\infty} \nu_{j}=\alpha_{4}-\alpha_{5}, \quad \sum_{0<\nu_{j}<\infty} \nu_{j}=\alpha_{4}, \\
& \sum_{0<\nu_{j}<\infty} 1=\alpha_{6}, \quad \sum_{\nu_{j}=\infty} 1=\alpha_{1}, \quad \sum_{\nu_{j} \leqq 0} 1=\alpha_{3}-\alpha_{6} .
\end{aligned}
$$


Moreover we have

$$
Q(\tau, s)=\tau^{\alpha_{1}} s^{\alpha_{2}} q_{0}(s) \prod_{0<\nu_{j}<\infty}\left(\tau-\lambda_{j}(s)\right) \prod_{\nu_{j} \leqq 0}\left(s^{-\nu_{j}} \tau-s^{-\nu_{j}} \lambda_{j}(s)\right) .
$$

Proof. Lemma A.1.2 easily follows from the Newton polygon of $Q(\tau, s)$.

Q.E.D.

Definition A.1.3. Let $\tau_{j}(s), j=1,2$, be Puiseux series. Then we define

$$
d\left(\tau_{1}, \tau_{2}\right)=\operatorname{order}\left(\tau_{1}(s)-\tau_{2}(s)\right)
$$

that is, $\tau_{1}(s)-\tau_{2}(s)=A s^{d\left(\tau_{1}, \tau_{2}\right)}(1+o(1))$ as $s \rightarrow+0, A \neq 0$.

The following lemma is obvious.

Lemma A.1.4. Let $\tau_{j}(s), j=1,2,3$, be Puiseux series. Then we have the following: (i) $d\left(\tau_{1}, \tau_{2}\right)=d\left(\tau_{2}, \tau_{1}\right)$. (ii) If $d\left(\tau_{1}, \tau_{2}\right)>d\left(\tau_{1}, \tau_{3}\right), d\left(\tau_{1}, \tau_{3}\right)=d\left(\tau_{2}, \tau_{3}\right)$. (iii) If $d\left(\tau_{1}, \tau_{2}\right)=d\left(\tau_{1}, \tau_{3}\right), d\left(\tau_{2}, \tau_{3}\right) \geqq d\left(\tau_{1}, \tau_{2}\right)$. (iv) $d\left(\tau_{1}+\tau_{3}, \tau_{2}+\tau_{3}\right)=d\left(\tau_{1}, \tau_{2}\right)$. (v) $d\left(\tau_{1}+a s^{\nu}, \tau_{2}\right)=\nu$ if $a \neq 0$ and $\nu<d\left(\tau_{1}, \tau_{2}\right)$. (vi) $d\left(\tau_{1}+a s^{\nu}, \tau_{2}\right)=\min \left(\nu, d\left(\tau_{1}, \tau_{2}\right)\right.$ ) for some $a$.

Let $\xi^{0}=(0,0,1)$ and $\vartheta=(1,0,0)$. Write $H(\tau, s, 1)$ and $S(\tau, s, 1)$ in the form

$$
\begin{aligned}
H(\tau, s, 1) & =H_{1}(\tau, s) R(\tau, s) A(\tau, s), & & A(0,0) \neq 0, \\
S(\tau, s, 1) & =S_{1}(\tau, s) R(\tau, s) B(\tau, s), & & B(0,0) \neq 0,
\end{aligned}
$$

where $H_{1}(\tau, s)$ and $S_{1}(\tau, s)$ are relatively prime. Let $\tau_{j}(s), 1 \leqq j \leqq r_{1}$, and $\lambda_{j}(s)$, $1 \leqq j \leqq r_{2}$, be the distinct roots of $H_{1}(\tau, s)=0$ and $S_{1}(\tau, s)=0$, respectively, such that order $\tau_{j}(s)>0$ and order $\lambda_{j}(s)>0$. Moreover let $\delta_{j}, 1 \leqq j \leqq r_{1}$, and $\gamma_{j}, 1 \leqq j$ $\leqq r_{2}$, be the multiplicities of the roots $\tau_{j}(s)$ and $\lambda_{j}(s)$, respectively. Here "the distinct roots" means that they are not identically equal. Hyperbolicity of $H(\xi)$ implies that the $\tau_{j}(s)$ are analytic and real-valued. Since $H_{1}$ and $S_{1}$ are relatively prime, we have

$$
d\left(\tau_{j}, \tau_{k}\right)<\infty \quad \text { for } j \neq k, \quad d\left(\lambda_{j}, \lambda_{k}\right)<\infty \quad \text { for } j \neq k, \quad d\left(\tau_{j}, \lambda_{k}\right)<\infty .
$$

Put $\eta(s)=\xi^{0} \pm s \xi^{1}+\sum_{j=1}^{\infty} a_{j} s^{\nu_{j}} \vartheta$, where $\xi^{1}=(0,1,0), a_{j} \in \boldsymbol{R} \backslash\{0\}$ and $\nu_{j} \in \boldsymbol{Q}, 0<\nu_{1}<$ $\nu_{2}<\cdots$. Consider the order of $I_{r}(\eta, l) \equiv S\left(\eta(s)-i \gamma s^{l} \vartheta\right) / H\left(\eta(s)-i \gamma s^{l} \vartheta\right)$ with respect to $s$, where $l>0, \gamma \in \boldsymbol{R} \backslash\{0\}$. It follows from Lemma A.1.2 that

$$
\begin{aligned}
\operatorname{order} I_{\gamma}(\eta, l)= & \alpha+\operatorname{order} \prod_{j=1}^{r_{2}}\left(\tau(s)-i \gamma s^{l}-\lambda_{j}( \pm s)\right)^{\gamma_{j}} \\
& -\operatorname{order} \prod_{j=1}^{r_{1}}\left(\tau(s)-i \gamma s^{l}-\tau_{j}( \pm s)\right)^{\delta_{j}},
\end{aligned}
$$

where $\tau(s)=\sum_{j=1}^{\infty} a_{j} s^{\nu_{j}}$ and $S_{1}(\tau, s)=s^{\alpha} \hat{S}_{1}(\tau, s), \hat{S}_{1}(\tau, 0) \neq 0$. It is easily seen that

$$
d\left(\tau(s)-i \gamma s^{l}, \tau_{j}( \pm s)\right) \leqq l \quad \text { for } \gamma \in R \backslash\{0\},
$$

and that 
(A.1.1)

$$
d\left(\tau(s)-i \gamma s^{l}, \lambda_{j}( \pm s)\right) \leqq l \quad \text { for some } \gamma \in R \backslash\{0\} .
$$

Choose $\gamma \in \boldsymbol{R} \backslash\{0\}$ so that (A.1.1) is fulfilled and put $\hat{\eta}(s ; l)=s^{-1} \eta\left(s^{1 / l}\right)$. Then we have

$$
\begin{aligned}
& \text { order } S(\hat{\eta}(s ; l)-i \gamma \vartheta) / H(\hat{\eta}(s ; l)-i \gamma \vartheta)=-n(\eta, l), \\
& n(\eta, l)=m^{\prime}-m+\rho(\eta, l) / l, \\
& \rho(\eta, l)=-\alpha+\sum_{j=1}^{r_{1}} \delta_{j} d\left(\tau(s)-i \gamma s^{l}, \tau_{j}( \pm s)\right)-\sum_{j=1}^{r_{2}} \gamma_{j} d\left(\tau(s)-i \gamma s^{l}, \lambda_{j}( \pm s)\right) .
\end{aligned}
$$

Lemma A.1.5.

$$
\begin{aligned}
n\left(H, S, \xi^{0}\right)=\sup \left\{n(\eta, l) ; \eta(s)=\xi^{0} \pm s \xi^{1}+\sum_{j=1}^{\infty} a_{j} S^{\nu_{j}} \vartheta,\right. \\
\left.l>0, a_{j} \in \boldsymbol{R} \backslash\{0\}, \nu_{j} \in \boldsymbol{Q} \text { and } 0<\nu_{1}<\nu_{2}<\cdots\right\} .
\end{aligned}
$$

Proof. Let $\hat{\gamma}(s)=s^{-1}\left(\xi^{0}+\sum_{j=1}^{\infty} s^{j / L} \eta^{j}\right) \in N_{\xi^{0}}, \eta^{j} \in R^{3}, L \in N$. Write $\hat{\eta}(s)=$ $a_{0}(s)\left(\xi^{0}+a_{1}(s) \xi^{1}+a_{2}(s) \vartheta\right)$, where $a_{0}(s)=s^{-1}(1+o(1))$ as $s \rightarrow+0$ and order $a_{j}(s)>0$, $j=1,2$. Assume that $a_{1}(s) \neq 0$. Then put $t=\left|a_{1}(s)\right|$. If $a_{1}(s)=c s^{\mu}(1+o(1)), c \neq 0$, as $s \rightarrow+0$, we have $s=c^{\prime} t^{1 / \mu}(1+o(1)) \equiv s(t), c^{\prime}>0$, as $t \rightarrow+0$. Thus

$$
\hat{\eta}(s(t))=a_{0}(s(t))\left(\xi^{0} \pm t \xi^{1}+\sum_{j=1}^{\infty} b_{j} t^{\nu} \vartheta\right) \equiv a_{0}(s(t)) \eta(t), \quad \text { if } t= \pm a_{1}(s),
$$

where $b_{j} \in \boldsymbol{R}$ and $\nu_{j} \in \boldsymbol{Q}, 0<\nu_{1}<\nu_{2}<\cdots$. Since

$$
\begin{aligned}
& |S(\hat{\eta}(s)-i \vartheta) / H(\hat{\eta}(s)-i \vartheta)| \\
& \quad=\left|a_{0}(s(t))^{m^{\prime}-m} S\left(\eta(t)-i a_{0}(s(t))^{-1} \vartheta\right) / H\left(\eta(t)-i a_{0}(s(t))^{-1} \vartheta\right)\right| \\
& \quad \leqq C\left|a_{0}(s(t))\right|^{\mid m^{\prime}-m} t^{-\rho(\eta, 1 / \mu)}(1+o(1)) \quad \text { as } t \rightarrow+0,
\end{aligned}
$$

we obtain

$$
n(H, S ; \hat{\eta}) \leqq n(\eta, 1 / \mu) .
$$

When $a_{1}(s) \equiv 0$, we can apply the above argument to $\hat{\eta}(s)$ replaced by $\hat{\eta}(s)+a \xi^{1}$. In fact, we have

$$
|S(\hat{\eta}(s)-i \vartheta) / H(\hat{\eta}(s)-i \vartheta)| \leqq C\left|S\left(\hat{\eta}(s)+a \xi^{1}-i \vartheta\right) / H\left(\hat{\eta}(s)+a \xi^{1}-i \vartheta\right)\right|
$$

for some $a \in \boldsymbol{R} \backslash\{0\}$, which shows that $n(H, S ; \hat{\eta}) \leqq n\left(H, S ; \hat{\eta}+a \xi^{1}\right)$. On the other hand it is obvious that

$$
n\left(H, S ; \xi^{0}\right) \geqq n(\eta, l) .
$$

(A.1.2) and (A.1.3) imply the lemma.

Q.E.D.

Lemma A.1.6. Let $\eta(s)=\xi^{0} \pm s \xi^{1}+\tau(s) \vartheta$, order $\tau(s)>0$. Assume that $d(\tau(s)$, $\left.\tau_{j}( \pm s)\right)<l, 1 \leqq j \leqq r_{1}$. Then we have $n(\eta, l) \leqq \max \left(m^{\prime}-m, n\left(\eta, l_{0}\right)\right)$, where

$$
l_{0}=\max _{1 \leqq j \leqq r_{1}} d\left(\tau(s), \tau_{j}( \pm s)\right) .
$$


Proof. From Lemma A.1.4 it follows that $\rho(\eta, l) \leqq \rho\left(\eta, l_{0}\right)$. In fact, we have

$$
d\left(\tau(s)-i \gamma s^{l}, \tau_{j}( \pm s)\right)=d\left(\tau(s)-i \gamma s^{l_{0}}, \tau_{j}( \pm s)\right)<l, \quad \gamma \in \boldsymbol{R},
$$

and

$$
\begin{aligned}
d\left(\tau(s)-i \gamma s^{l}, \lambda_{j}( \pm s)\right) & =\min \left(l, d\left(\tau(s), \lambda_{j}( \pm s)\right)\right), \\
d\left(\tau(s)-i \gamma s^{l_{0}}, \lambda_{j}( \pm s)\right) & =\min \left(l_{0}, d\left(\tau(s), \lambda_{j}( \pm s)\right)\right)
\end{aligned}
$$

for some $\gamma \in \boldsymbol{R} \backslash\{0\}$. If $\rho\left(\eta, l_{0}\right) \leqq 0$, then $n(\eta, l) \leqq m^{\prime}-m$. On the other hand we have $n(\eta, l) \leqq n\left(\eta, l_{0}\right)$ if $\rho\left(\eta, l_{0}\right) \geqq 0$.

Q.E.D.

If $H_{1}(0,0) \neq 0$, that is, $H_{1}(\tau, s) \equiv 1$, then $n\left(H, S ; \xi^{0}\right)=m^{\prime}-m$. Therefore we consider only the case $H_{1}(0,0)=0$. Then we have $n\left(H, S ; \xi^{0}\right)>m^{\prime}-m$. For it is easy to see that $n(\eta, l) \rightarrow m^{\prime}-m+\delta_{1}$ as $l \rightarrow \infty$ if $\eta(s)=\xi^{0}+s \xi^{1}+\tau_{1}(s) \vartheta$. By Lemma A.1.6 we have

$$
\sup _{l>0} n(\eta, l)=\sup _{0<l \leqq l_{0}} n(\eta, l),
$$

where $\eta(s)=\xi^{0} \pm s \xi^{1}+\tau(s) \vartheta$ and $l_{0}$ is defined by (A.1.4), if $\sup _{l>0} n(\eta, l)>m^{\prime}-m$. If $l \leqq l_{0}$, then $\tau(s)$ can be written in the form

$$
\tau(s)=\sum_{k<l, k \in N} a_{j_{0} k}( \pm s)^{k}+s^{k_{0}} \sum_{k=1}^{\infty} a_{k}^{\prime} s^{\nu_{k}^{\prime}},
$$

where $1 \leqq j_{0} \leqq r_{1}, \tau_{j_{0}}(s)=\sum_{k=1}^{\infty} a_{j_{0}} s^{k}, k_{0}=\max \{j \in Z ; k<l\}(\geqq 0), 0<\nu_{1}^{\prime}<\nu_{2}^{\prime}<\cdots$, $k_{0}+\nu_{1}^{\prime} \geqq l$. Therefore we have

$$
\begin{aligned}
& d\left(\tau(s)-i \gamma s^{l}, \tau_{j}( \pm s)\right)=d\left(\tau_{j_{0}}(s)-i \gamma s^{l}, \tau_{j}(s)\right), \quad \gamma \in \boldsymbol{R} \backslash\{0\}, \\
& d\left(\tau(s)-i \gamma s^{l}, \lambda_{j}( \pm s)\right)=d\left(\tau_{j_{0}}(s)-i \gamma s^{l}, \lambda_{j}(s)\right) \quad \text { for some } \gamma \in \boldsymbol{R} \backslash\{0\} .
\end{aligned}
$$

If we put $\eta_{j_{0}}(s)=\xi^{0}+s \xi^{1}+\tau_{j_{0}}(s) \vartheta$, we have $n(\eta, l)=n\left(\eta_{j_{0}}(s), l\right)$. Thus it suffices to choose $\eta_{j}(s) \equiv \xi^{0}+s \xi^{1}+\tau_{j}(s) \vartheta, 1 \leqq j \leqq r_{1}$, as $\eta(s)$ in order to calculate $n(H, S$;

$\left.\xi^{0}\right)$. Define

$$
\begin{array}{ll}
d_{i j}=d\left(\tau_{i}, \tau_{j}\right) & \text { for } 1 \leqq i, j \leqq r_{1}, \\
d_{i j+r_{1}}=d\left(\tau_{i}, \lambda_{j}\right) & \text { for } 1 \leqq i \leqq r_{1} \text { and } 1 \leqq j \leqq r_{2}, \\
\delta_{j+r_{1}}=-\gamma_{j} & \text { for } 1 \leqq j \leqq r_{2} .
\end{array}
$$

Moreover put

$$
n_{j}(l)=\rho\left(\eta_{j}(s), l\right) / l=\left(\sum_{k=1}^{r_{1}+r_{2}} \delta_{k} \min \left(d_{j k}, l\right)-\alpha\right) / l, \quad 1 \leqq j \leqq r_{1} .
$$

The set $\left\{d_{i j} ; 1 \leqq j \leqq r_{1}+r_{2}\right\}$ can be written as follows:

$$
\begin{aligned}
& \left\{d_{i j} ; 1 \leqq j \leqq r_{1}+r_{2}\right\}=\left\{l_{i j} ; 1 \leqq j \leqq q_{i}\right\}, \quad 1 \leqq i \leqq r_{1}, \\
& 0<l_{i 1}<l_{i 2}<\cdots<l_{i q_{i}}=\infty
\end{aligned}
$$

Put 


$$
\beta_{i j}=\sum_{d_{i k}=l_{i j}} \delta_{k}, \quad \beta_{i 0}=0, \quad I_{i j}=\sum_{k=1}^{j} \beta_{i k} l_{i k}, \quad I_{i 0}=0, \quad 1 \leqq i \leqq r_{1}, \quad 1 \leqq j \leqq q_{i} .
$$

Then we have

$$
n_{i}(l)=\left(I_{i i(l)}-\alpha\right) / l+\sum_{k=i(l)+1}^{q_{i}} \beta_{i k},
$$

where $i(l)=\max \left\{j \in Z ; l_{i j} \leqq l\right\}, l_{i 0}=0$.

\section{Lemma A.1.7.}

$$
\sup _{l>0} n_{i}(l)=\max \left\{n_{i}(l) ; l=d_{i j}, 1 \leqq j \leqq r_{1}\right\}, \quad 1 \leqq j \leqq r_{1},
$$

where $n_{i}(\infty)=\delta_{i}$.

Proof. Assume that $d_{i j_{0}} \equiv l_{i j_{1}}<l<d_{i j_{2}} \equiv l_{i j_{3}}, 0 \leqq j_{0} \leqq r_{1}-1,1 \leqq j_{2} \leqq r_{1}$, and that $\left(d_{i j_{0}}, d_{i j_{2}}\right)$ does not contain $d_{i j}, 1 \leqq j \leqq r_{1}$, where $d_{i 0}=0, d_{i i}=\infty$. Then we have

$$
n_{i}\left(l_{i j_{1}}\right)-n_{i}(l)=\left(1 / l_{i j_{1}}-1 / l\right)\left(I_{i i(l)}-\alpha\right)+\sum_{k=j_{1}+1}^{i(l)}\left(\beta_{i k}-\beta_{i k} l_{i k} / l_{i j_{1}}\right)
$$

$$
n_{i}\left(l_{i j_{3}}\right)-n_{i}(l)=\left(1 / l_{i j_{3}}-1 / l\right)\left(I_{i i(l)}-\alpha\right)+\sum_{\substack{j_{3}-1 \\ k=i(l)+1}}\left(\beta_{i k} l_{i k} / l_{i j_{3}}-\beta_{i k}\right)^{\text {for } j_{0} \geqq 1 \text {, }}
$$

It is obvious that

$$
\sum_{k=j_{1}+1}^{i(l)}\left(\beta_{i k}-\beta_{i k} l_{i k} / l_{i j_{1}}\right), \sum_{k=i(l)+1}^{j_{3}-1}\left(\beta_{i k} l_{i k} / l_{i j_{3}}-\beta_{i k}\right) \geqq 0 .
$$

If $l_{i j_{3}}=\min \left\{d_{i j} ; 1 \leqq j \leqq r_{1}\right\}$, that is, $j_{0}=0$, then $n_{i}\left(l_{i j_{3}}\right) \geqq n_{i}(l)$. In fact, $I_{i i(l)}-\alpha \leqq 0$, since $\beta_{i k} \leqq 0$ if $k \leqq i(l)<j_{3}$. Moreover we have for $j_{0} \geqq 1$

$$
\begin{array}{ll}
n_{i}\left(l_{i j_{1}}\right) \geqq n_{i}(l) & \text { if } I_{i i(l)}-\alpha \geqq 0, \\
n_{i}\left(l_{i j_{3}}\right) \geqq n_{i}(l), & \text { otherwise. }
\end{array}
$$

Thus the proof of the lemma is complete.

Q.E.D.

Theorem A.1.8. We have

$$
n\left(H, S ; \xi^{0}\right)=m^{\prime}-m+\max \left\{n_{i}(l) ; l=d_{i j}, 1 \leqq i, j \leqq r_{1}\right\} .
$$

Moreover if $n\left(H, S ; \xi^{0}\right)=m^{\prime}-m+n_{i_{0}}\left(d_{i_{0} j_{0}}\right), 1 \leqq i_{0}, j_{0} \leqq r_{1}$, then $I_{i_{0} j_{1}}-\alpha \geqq 0$ and $I_{i_{0} j_{1}-1}-\alpha \leqq 0$, where $d_{i_{0} j_{0}}=l_{i_{0} j_{1}}$.

REMARK. If $n\left(H, S ; \xi^{0}\right)=m^{\prime}-m+n_{i}(\infty)=m^{\prime}-m+\delta_{i}$, there exists $\xi^{1} \in \boldsymbol{R}^{n}$ such that $n(H, S ; \hat{\eta})=m^{\prime}-m+\delta_{i}$, where $\hat{\eta}(s)=s^{-1} \xi^{1}$.

Proof. (A.1.6) easily follows from Lemma A.1.7. If we put $l=l_{i_{0} j_{1}}$ in (A.1.5), it follows that $I_{i_{0} j_{1}}-\alpha \geqq 0$. The same argument as in the proof of Lemma A.1.7 gives 


$$
n_{i_{0}}\left(l_{i_{0} j_{1}}\right)-n_{i_{0}}\left(l_{i_{0} j_{1}-1}\right)=\left(I_{i_{0} j_{1}-1}-\alpha\right)\left(1 / l_{i_{0} j_{1}}-1 / l_{i_{0} j_{1}-1}\right),
$$

which implies that $I_{i_{0} j_{1}-1}-\alpha \leqq 0$.

Q.E.D.

By Theorem A.1.8 we obtain the following procedure to determine $n\left(H, S ; \xi^{0}\right)$ when $n=3$ :

Procedure. 1. Make a change of variables so that $\xi^{0}=(0,0,1)$ and $\vartheta=$ $(1,0,0)$.

2. Write

$$
\begin{aligned}
H(\tau, s, 1) & =H_{1}(\tau, s) R(\tau, s) A(\tau, s), & & A(0,0) \neq 0, \\
S(\tau, s, 1) & =S_{1}(\tau, s) R(\tau, s) B(\tau, s), & & B(0,0) \neq 0, \\
S_{1}(\tau, s) & =s^{\alpha} \hat{S}_{1}(\tau, s), & \hat{S}_{1}(\tau, 0) \neq 0, &
\end{aligned}
$$

where $H_{1}(\tau, s)$ and $S_{1}(\tau, s)$ are relatively prime.

3. Let $\tau_{j}(s), 1 \leqq j \leqq r_{1}$, and $\lambda_{j}(s), 1 \leqq j \leqq r_{2}$, be the distinct roots of $H_{1}(\tau, s)=0$ and $S_{1}(\tau, s)=0$, respectively, such that order $\tau_{j}(s)>0$ and order $\lambda_{j}(s)>0$. Moreover let $\delta_{j}, 1 \leqq j \leqq r_{1}$, and $-\delta_{j+r_{1}}, 1 \leqq j \leqq r_{2}$, be the multiplicities of the roots $\tau_{j}(s)$ and $\lambda_{j}(s)$, respectively.

4. Put $d_{i j}=d\left(\tau_{i}, \tau_{j}\right)$ for $1 \leqq i, j \leqq r_{1}$ and $d_{i j+r_{1}}=d\left(\tau_{i}, \lambda_{j}\right)$ for $1 \leqq i \leqq r_{1}, 1 \leqq j \leqq$ $r_{2}$, where $d\left(\tau_{i}, \tau_{j}\right)=\operatorname{order}\left(\tau_{i}(s)-\tau_{j}(s)\right)$.

5. Write $\left\{d_{i j} ; 1 \leqq j \leqq r_{1}+r_{2}\right\}=\left\{l_{i j} ; 1 \leqq j \leqq q_{i}\right\}, 0<l_{i 1}<l_{i 2}<\cdots<l_{i q_{i}}=\infty$.

6. Put $\beta_{i j}=\sum_{d_{i k}=l_{i j}} \delta_{k}, I_{i j}=\sum_{k=1}^{j} \beta_{i k} l_{i k}$.

7. Let $i(k), 1 \leqq k \leqq p_{i}$, be integers such that $I_{i i(k)}-\alpha \geqq 0, I_{i i(k)-1}-\alpha \leqq 0$ and $l_{i i(k)}=d_{i j}$ for some $j$ with $1 \leqq j \leqq r_{1}$.

8. Then we have

$$
n\left(H, S ; \xi^{0}\right)=m^{\prime}-m+\max \left\{\left(I_{i i(k)}-\alpha\right) / l_{i i(k)}+\sum_{j=i(k)+1}^{q_{i}} \beta_{i j} ; 1 \leqq i \leqq r_{1}, 1 \leqq k \leqq p_{i}\right\},
$$

where $\left(I_{i i(k)}-\alpha\right) / l_{i i(k)}+\sum_{j=i(k)+1}^{q_{i}} \beta_{i j}=\delta_{i}$ if $l_{i i(k)}=\infty$.

\section{§ A.2. Examples}

ExAmple A.2.1. Let $P(\xi)=\left(\xi_{1}^{2}-\xi_{2}^{2}+2 \xi_{1} \xi_{3}\right)\left(\xi_{1}^{2}-2 \xi_{2}^{2}+2 \xi_{1} \xi_{3}\right)+\xi_{2} \xi_{3}^{2}$ and $\xi^{0}=$ $(0,0,1)$. Then we obtain

$$
\begin{aligned}
& \alpha=1, \quad \tau_{1}(s)=s^{2} / 2+\cdots, \quad \tau_{2}(s)=s^{2}+\cdots, \\
& \left(l_{i j}\right)=\left(\begin{array}{ll}
2 & \infty \\
2 & \infty
\end{array}\right), \quad\left(\beta_{i j}\right)=\left(\begin{array}{ll}
1 & 1 \\
1 & 1
\end{array}\right), \quad\left(I_{i j}\right)=\left(\begin{array}{ll}
2 & \infty \\
2 & \infty
\end{array}\right), \quad\left(I_{i j}-\alpha\right)=\left(\begin{array}{ll}
1 & \infty \\
1 & \infty
\end{array}\right) .
\end{aligned}
$$

Thus $n\left(P_{4}, Q_{3} ; \xi^{0}\right)=-1+1 / 2+1=1 / 2$ and $\kappa\left(\xi^{0}\right)=(1+1 / 2) /(1 / 2)=3$.

EXAMPLE A.2.2. Let $P(\xi)=\left(\xi_{1}^{2}-\xi_{2}^{2}+2 \xi_{1} \xi_{3}\right)\left(\xi_{1}^{2}-2 \xi_{2}^{2}+2 \xi_{1} \xi_{3}\right)\left(\xi_{1}^{2}-3 \xi_{2}^{2}+2 \xi_{1} \xi_{3}\right)+$ $\xi_{2}^{3} \xi_{3}^{2}$. If $\xi^{0}$ is not parallel to $(0,0,1)$ and $(2,0,-1)$, then $\left(\partial / \partial \xi_{1}\right) P_{6}\left(\xi^{0}\right) \neq 0$. Thus 
it follows from Lemma 1.2.3 that $n\left(P_{6}, Q_{5} ; \xi^{0}\right)=0$ if $\xi^{0}$ is not parallel to $(0,0,1)$ and $(2,0,-1)$. Let $\xi^{0}=(0,0,1)$. Then we obtain

$$
\begin{aligned}
& \alpha=3, \quad \tau_{1}(s)=s^{2} / 2+\cdots, \quad \tau_{2}(s)=s^{2}+\cdots, \quad \tau_{3}(s)=3 s^{2} / 2+\cdots, \quad\left(\begin{array}{ll}
2 & \infty \\
2 & \infty \\
2 & \infty
\end{array}\right), \quad\left(\beta_{i j}\right)=\left(\begin{array}{ll}
2 & 1 \\
2 & 1
\end{array}\right), \quad\left(I_{i j}\right)=\left(\begin{array}{ll}
4 & \infty \\
4 & \infty \\
4 & \infty
\end{array}\right), \quad\left(I_{i j}-\alpha\right)=\left(\begin{array}{ll}
1 & \infty \\
1 & \infty \\
1 & \infty
\end{array}\right) .
\end{aligned}
$$

Thus $n\left(P_{6}, Q_{5} ; \xi^{0}\right)=-1+1 / 2+1=1 / 2$. When $\xi^{0}=(2,0,-1)$, we have also $n\left(P_{6}, Q_{5} ; \xi^{0}\right)=1 / 2$. Therefore $\kappa_{0}=1 / \delta(P)=3$. We note that $P_{\xi}$ is hyperbolic with respect to $\vartheta$ for $\xi \in \boldsymbol{R}^{3} \backslash\{0\}$, although $P$ is not hyperbolic with respect to $\vartheta$.

ExAmple A.2.3. Let $P(\xi)=\left(\xi_{1}^{2}-\xi_{2}^{2}+2 \xi_{1} \xi_{3}\right)\left(\xi_{1}^{2}-2 \xi_{2}^{2}+4 \xi_{1} \xi_{3}\right)+2 \xi_{1} \xi_{3}^{2}$ and $\xi^{0}=$ $(0,0,1)$. Then we obtain

$$
\begin{aligned}
& \alpha=0, \quad \tau_{1}(s)=s^{2} / 2-s^{4} / 8+\cdots, \quad \tau_{2}(s)=s^{2} / 2-s^{4} / 16+\cdots, \\
& \lambda_{1}(s) \equiv 0, \quad\left(l_{i j}\right)=\left(\begin{array}{lll}
2 & 4 & \infty \\
2 & 4 & \infty
\end{array}\right), \quad\left(\beta_{i j}\right)=\left(\begin{array}{lll}
-1 & 1 & 1 \\
-1 & 1 & 1
\end{array}\right), \quad\left(I_{i j}\right)=\left(\begin{array}{lll}
-2 & 2 & \infty \\
-2 & 2 & \infty
\end{array}\right), \\
& \left(I_{i j}-\alpha\right)=\left(\begin{array}{lll}
-2 & 2 & \infty \\
-2 & (2) & \infty
\end{array}\right) .
\end{aligned}
$$

Thus $n\left(P_{4}, Q_{3} ; \xi^{0}\right)=-1+2 / 4+1=1 / 2$ and $\kappa\left(\xi^{0}\right)=3$.

\section{References}

[1] M. F. Atiyah, R. Bott and L. Gårding, Lacunas for hyperbolic differential operators with constant coefficients I, Acta Math., 124, 109-189 (1970).

[2] J. J. Duistermaat, Fourier integral operators, Lecture notes, Courant Inst. Math. Sci., New York Univ., 1973.

[3] J. L. Dunn, A sufficient condition for hyperbolicity of partial differential operators with constant coefficient principal part, Trans. Amer. Math. Soc., 201, 315-327 (1975).

[4] L. Gårding, Linear hyperbolic partial differential equations with constant coeficients, Acta Math., 85, 1-62 (1950).

[5] L. Gårding, Some trends and problem in linear partial differential equations, Proc. Int. Congr. Math. Edinburgh, 87-102 (1958).

[6] E. A. Gorin, Asymptotic properties of polynomials and algebraic functions of several variables, Uspehi Mat. Nauk, 16, 93-119 (1961). (Russian; English translation in Russian Math. Surveys.)

[7] L. Hörmander, Linear partial differential operators, Springer-Verlag, BerinGöttingen-Heidelberg, 1963.

[ 8 ] L. Hörmander, Uniqueness theorems and wave front sets for solutions of linear differential equations with analytic coeffeients, Comm. Pure Appl. Math., 24, 671-704 (1971).

[ 9 ] L. Hörmander, Fourier integral operators, I, Acta Math., 127, 79-183 (1971).

[10] V. Ja. Ivrii, Correctness of the Cauchy problem in Gevrey classes for nonstrictly hyperbolic operators, Mat. Sb., 96, 390-413 (1975). (Russian; English trans- 
lation in Math. USSR Sb.)

[11] V. Ja. Ivrii, Conditions for correctness in Gevrey classes of the Cauchy problem for weakly hyperbolic equations, Sib. Mat. Zh., 17, 547-563 (1976).

[12] V. Ja. Ivrii and V. M. Petkov, Necessary conditions for the Cauchy problem for non-strictly hyperbolic equations to be well-posed, Uspehi Mat. Nauk, 29, 3-70 (1974). (Russian; English translation in Russian Math. Surveys.)

[13] H. Komatsu, Ultradistributions, I, Structure theorems and a characterization, J. Fac. Sci. Univ. Tokyo, Sect. IA, 20, 25-105 (1973).

[14] H. Komatsu, Ultradistributions, II, The kernel theorem and ultradistributions with support in a submanifold, J. Fac. Sci. Univ. Tokyo, Sect. IA, 24, 607-628 (1977).

[15] E. Larsson, Generalized hyperbolicity, Ark. Mat., 7, 11-32 (1967).

[16] P. D. Lax, Asymptotic solutions of oscillatory initial value problems, Duke Math. J., 24, 627-646 (1957).

[17] S. Mizohata, Some remarks on the Cauchy problem, J. Math. Kyoto Univ., 1, 109-127 (1961).

[18] C. Roumieu, Ultra-distributions définies sur $\boldsymbol{R}^{n}$ et sur certaines clsases de variétés différentiables, J. Analyse Math., 10, 153-192 (1962-1963).

[19] S. L. Svensson, Necessary and sufficient conditions for the hyperbolicity of polynomials with hyperbolic principal part, Ark. Mat., 8, 145-162 (1969).

[20] S. Wakabayashi, Propagation of singularities for hyperbolic operators with constant coefficient principal part, Tsukuba J. Math., 2, 91-107 (1978).

\section{Institute OF MATHEMATICS \\ UNIVERSITY OF TSUKUBA \\ IBARAKI 305}

